\title{
MONITORING CONSERVATIVE RETROFITS \\ IN \\ SINGLE FAMILY BUILDINGS
}

DE-FG01-90CE21002

\section{Final Technical Report}

\author{
Presented to: \\ Mr. Ernest Freeman, Jr. \\ U.S. Department of Energy \\ Buildings and Commercial systems \\ 100 Independence Avenue, S.W. \\ Washington, D.C. 20585 \\ December 6, 1992
}

\author{
by \\ Carl S. Richardson \\ Florida Solar Energy Center \\ 300 State Road 401 \\ Cape Canaveral, Florida 32920 \\ DISCLAIMER
}

This report was prepared as an account of work sponsored by an agency of the United States Government. Neither the United States Government nor any agency thereof, nor any of their employees, makes any warranty, express or implied, or assumes any legal liability or responsiSility for the accuracy, completeness, or usefulness of any information, apparatus, product, or pricess disclosed, or represents that its use would not infringe privately owned rights. Reference herein to any specific commercial product, process, or service by trade name, trademark, manufacturer, or otherwise does not necessarily constitute or imply its endorsement, recommendation, or favoring by the United States Government or any agency thereof. The views and opinions of authors expressed herein do not necessarily state or reflect those of the United States Government or any agency thereof. 
TABLE OF CONTENTS

1. Objective . . . . . . . . . . . . . 1

2. Retrofits studied . . . . . . . . . . . 1

3. Site selection . . . . . . . . . . . 1

4. Data acquired . . . . . . . . . . . 2

5. Support documentation . . . . . . . . . 2

\section{APPENDICES}

Appendix A . . . . . . . . . . . . . . 3 Participants

Appendix B . • • . . . . . . . . . . . . . . 5 Significant dates

Appendix C . . . . . . . . . . . . . . 15 Sample data set

Appendix D . . . . . . . . . . . . . 20 Pre-retrofit audit data sheets Post-retrofit audit data sheets

Appendix E . . . . . . . . . . . . . . . . . . . . . . . . . Power usage history 


\section{Objective}

There has been much progress in understanding the effects of energy conservation retrofits in heating climates. Studies in the Pacific Northwest, Wisconsin, New York, and other northern areas have demonstrated that audit prioritized retrofits can effect significant savings in energy consumption over the use of a priority list for initiating retrofits. There has been little data generated, however, in climates in which air conditioning, and not heating, is the primary energy user.

Measured field tests of air-conditioning climate upgrades are necessary to provide the data which would allow governmental agencies, utilities, and homeowners to make optimal usage of upgrade monies to effect the maximum energy savings without negatively affecting the comfort conditions of the residence.

This study has provided detailed before-and after information on the ambient and comfort conditions in nine single family buildings, and on the energy consumption of those buildings, for one or more energy conservation retrofits. The data were recorded in such a manner that as well as being able to determine the savings from the retrofits and the influence these retrofits have on the comfort conditions of the residence, the effects of the retrofits on time-of-day usage are also determinable.

2. Retrofits studied

The retrofits considered for inclusion in the study were some of those which Florida Power \& Light, a co-sponsor of the study, included in their homeowner energy audit and rebate program. More specifically, the retrofits actually performed at the participating houses were:

*) Central air conditioner upgrade to more efficient, i.e.>SEER 11, unit;

b) Upgrade of attic insulation to at least R19;

c) Installation of a hot water heat recovery unit on the central air conditioner.

\section{Site selection}

Site selection was made through the cooperation of FP\&L energy auditors. In instances where the house being audited was recommended for one or more of the studied retrofits the auditor would advise the homeowner of the study. Interested homeowners then contacted the investigator for more information and to schedule an interview at which the investigator would determine the suitability of the house for 
the study. Ten houses were obtained for the study, but one homeowner dropped out of the project (and sold the house) before the retrofit was installed.

\section{Data Acquired}

The conditions recorded for this study were:

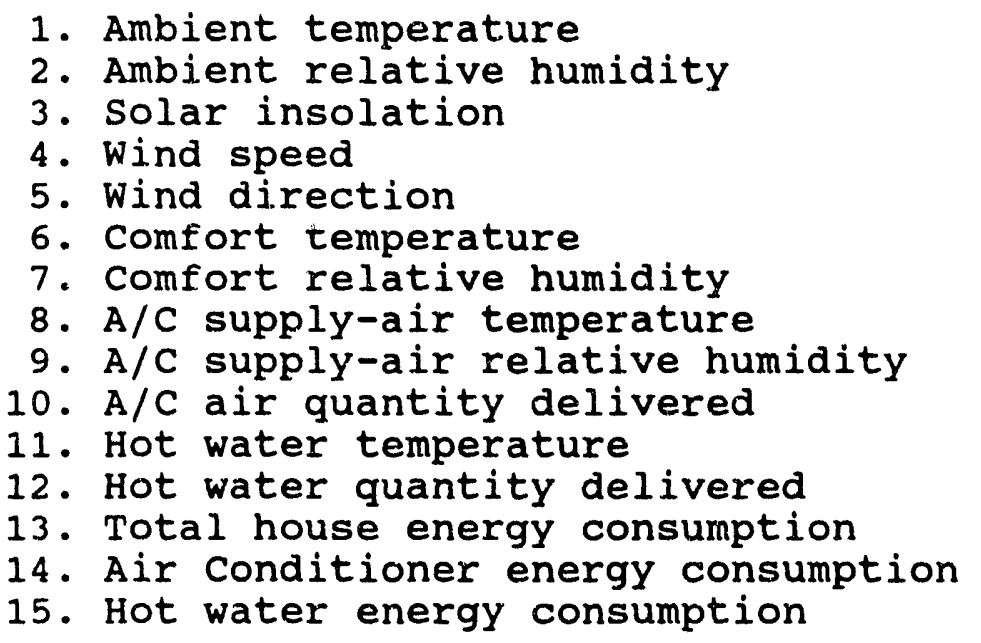

The conditions were continuously monitored, and recorded on fifteen minute intervals, the data being averaged or totalized as appropriate. An ASCII comma delineated file was made for each 24 hour period and saved in the on-site computer, each file named with the experimental site designation and the date the data were acquired.

Data were archived at the Florida Solar Energy Center, and were also forwarded to the Oak Ridge National Laboratory monthly.

\section{Support Documentation}

A table of participant's names, site addresses and retrofit is Appendix A.

Significant dates - beginning of data acquisition, dates and type of retrofit, observed problems, etc. - and appropriate comments are presented in Appendix B.

An example of a day of data and an annotated data set are presented in Appendix $C$.

Pre-retrofit Audit Data Sheets and Post-retrofit Audit Data Sheets are Appendix D.

Usage history information is duplicated in Appendix E. 
APPENDIX A

PARTICIPANTS 


\begin{tabular}{|c|c|c|c|}
\hline cod & & Participant & Retrofit(s) \\
\hline WL & 1 & $\begin{array}{l}\text { Wayne A. Legros } \\
399 \text { Charlotta Ave S.E. } \\
\text { Palm Bay, FL } 32909\end{array}$ & $\begin{array}{l}\text { A/C upgrade } \\
\mathrm{H} / \mathrm{W} \text { heat recovery }\end{array}$ \\
\hline RH & 2 & $\begin{array}{l}\text { Rose Hirt } \\
2817 \text { Kensington Dr. } \\
\text { Melbourne, FL } 32935\end{array}$ & $\begin{array}{l}\text { A/C upgrade } \\
\text { H/W heat recovery } \\
\text { Ceiling insulation }\end{array}$ \\
\hline WS & 3 & $\begin{array}{l}\text { William H. Schick } \\
1688 \text { Shore Drive } \\
\text { Merritt Island, FL } 32952\end{array}$ & Ceiling insulation \\
\hline PP & 4 & $\begin{array}{l}\text { Peter Postma } \\
311 \text { Cocoa Ave } \\
\text { Indialantic, FL } 32903\end{array}$ & Ceiling insulation \\
\hline$R R$ & 5 & $\begin{array}{l}\text { Richard E. Rainsberger } \\
209 \text { Flamingo Lane } \\
\text { Melbourne Beach, FL } 32951\end{array}$ & $\begin{array}{l}\text { A/C upgrade } \\
\text { Ceiling insulation }\end{array}$ \\
\hline $\mathrm{BC}$ & 6 & $\begin{array}{l}\text { Bernard J. Cohn } \\
154 \text { Abalone Road } \\
\text { Palm Bay, FL } 32907\end{array}$ & A/C upgrade \\
\hline sc & 7 & $\begin{array}{l}\text { Steve Chilberg } \\
2036 \text { Abalone Street } \\
\text { Indialantic, FL } 32903\end{array}$ & $\begin{array}{l}\text { Withdrew before } \\
\text { retrofit }\end{array}$ \\
\hline EK & 8 & $\begin{array}{l}\text { Edward Kizak } \\
216 \text { Melbourne Ave. } \\
\text { Indialantic, FL } 32903\end{array}$ & A/C Upgrade \\
\hline WH & 9 & $\begin{array}{l}\text { William Handley } \\
2200 \text { Ohio Street } \\
\text { West Melbourne, FL } 32904\end{array}$ & $\begin{array}{l}\text { A/C Upgrade } \\
\text { H/W heat recovery }\end{array}$ \\
\hline $\mathrm{JP}$ & 10 & $\begin{array}{l}\text { Jon Peterson } \\
3081 \text { Grace Street } \\
\text { West Melbourne, FL } 32904\end{array}$ & Ceiling Insulation \\
\hline
\end{tabular}


APPENDIX B

SIGNIFICANT DATES 


\section{MONITORING CONSERVATIVE RETROFITS}

IN SINGLE FAMILY BUILDINGS

SITE CODE: WL

SITE \#: $\quad 1$

MONITORING BEGAN: 6/13/91

RETROFIT \#1: A/C UPGRADE DATE 7/9/91

RETROFIT \#2: H/W HEAT RECOVERY DATE 8/7/91

RETROFIT \#3: DATE

TOGGLES :

\#1: SWIMMING POOL PUMP

\#2: DOMESTIC WATER PUMP

COMMENTS :

DATA ACQUISITION ENDS $12 / 22 / 91$ 


\section{MONITORING CONSERVATIVE RETROFITS}

IN SINGLE FAMILY BUILDINGS

SITE CODE: $\quad$ RH

SITE \# : $\quad 2$

MONITORING BEGAN: $6 / 25 / 91$

RETROFIT \#1: A/C UPGRADE DATE 7/23/91

RETROFIT \#2: H/W HEAT RECOVERY DATE 8/14/91

RETROFIT \#3: INSULATION DATE $7 / 26 / 91$

TOGGLES :

\#1: NONE

\#2:

COMMENTS :

DATALOGGER FAILURE 9/4/91, REPLACED 10/3

THP METER FAILURE $9 / 4 / 91-10 / 8 / 91$ 
MONITORING CONSERVATIVE RETROFITS

IN SINGLE FAMILY BUILDINGS

SITE CODE: WS

SITE \# : $\quad 3$

MONITORING BEGAN: $7 / 24 / 91$

RETROFIT \#1: INSULATION TO R30 DATE 8/20/91

RETROFIT \#2: DATE

RETROFIT \#3: DATE

TOGGLES :

\#1: NONE

\#2:

COMMENTS :

WIND DIRECTION FAILURE 8/15, NOT REPLACED

HOT WITER POWER METER FAILURE 8/24, REPLACED 9/4

HOT WATER POWER METER FAILURE $9 / 6$, REPLACED $9 / 12$

HOT WATER POWER METER FAILURE REPLACED $₫ 0 / 24$

SWIMMING POOL PUMP ON TIMER - 8:00 A.M. UNTIL 6:00 P.M. 


\section{MONITORING CONSERVATIVE RETROFITS}

IN SINGLE FAMILY BUILDINGS

$\begin{array}{ll}\text { SITE CODE: } & \text { PP } \\ \text { SITE \# : } & 4\end{array}$

MONITORING BEGAN: 7/23/91

RETROFIT \#1: INSULATION TO R19 DATE 9/5/91

RETROFIT \#2: DATE

RETROFIT \#3: DATE

TOGGLES :

\#1: NONE

\#2:

COMMENTS :

NO POWER, H2O FLOW UNTIL $8 / 1$

NO POWER, H2O FLOW $9 / 11-9 / 16$ 


\section{MONITORING CONSERVATIVE RETROFITS \\ IN SINGLE FAMILY BUILDINGS}

SITE CODE: $\quad$ RR

SITE \# : $\quad 5$

MONITORING BEGAN: $7 / 31 / 91$

RETROFIT \#1: INSULATION TO R30 DATE 9/5/91

RETROFIT \#2: A/C UPGRADE DATE $9 / 6 / 91$

RETROFIT \#3: DATE

TOGGLES :

\#1: NONE

\#2:

COMMENTS :

WIND DIRECTION FAILURE $8 / 20$, NOT REPAIRED

NO A/C POWER, AIR FLOW INCORRECT 9/6 - 9/10

OCCUPANTS ON VACATION $9 / 14-9 / 29$ 


\section{MONITORING CONSERVATIVE RETROFITS \\ IN SINGLE FAMILY BUILDINGS}

$\begin{array}{ll}\text { SITE CODE: } & \text { BC } \\ \text { SITE \# : } & 6\end{array}$

MONITORING BEGAN: $8 / 6 / 91$

RETROFIT \#1: A/C UPGRADE

DATE $\quad 9 / 6 / 91$

RETROFIT \#2:

DATE

RETROFIT \#3:

DATE

TOGGLES :

\#1: NONE

\#2:

COMMENTS :

TROUBLE FREE 
MONITORING CONSERVATIVE RETROFITS

IN SINGLE FAMILY BUILDINGS

SITE CODE: $\quad$ EK

SITE \#: 8

MONITORING BEGAN: $8 / 27 / 91$

RETROFIT \#1: A/C UPGRADE DATE 10/14/91

RETROFIT \#2: $\quad$ DATE

RETROFIT \#3: $\quad$ DATE

TOGGLES:

\#1: NONE

\#2:

COMMENTS :

TOTAL HOUSE POWER QUESTIONABLE UNTIL $9 / 17$

NO DATA $10 / 9$ - 10/14 DURING A/C REPLACEMENT 


\section{MONITORING CONSERVATIVE RETROFITS \\ IN SINGLE FAMILY BUILDINGS}

$\begin{array}{ll}\text { SITE CODE: } & \text { WH } \\ \text { SITE \# : } & 9\end{array}$

MONITORING BEGAN: $9 / 6 / 91$

RETROFIT \#1: A/C UPGRADE DATE 10/21/91

RETROFIT \#2: H/W HEAT RECOVERY DATE 11/6/91

RETROFIT \#3: DATE

TOGGLES :

\#1: DOMESTIC WATER PUMP

\#2:

COMMENTS :

NO POWER METERS UNTIL $9 / 14$

NO DATA $10 / 9$ - 10/14 DURING A/C REPLACEMENT 


\section{MONITORING CONSERVATIVE RETROFITS IN SINGLE FAMILY BUILDINGS}

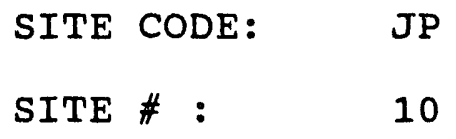

TOGGLES :

\#1: POOL PUMP

\#2:

COMMENTS :

AIR FLOW INTERFERENCE UNTIL $9 / 20$

"AFTER" DATA DURING 1992 COOLING SEASON 


\section{APPENDIX C}

SAMPLE DATA SET 


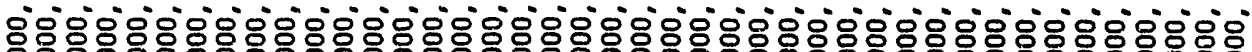

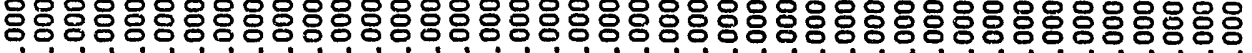

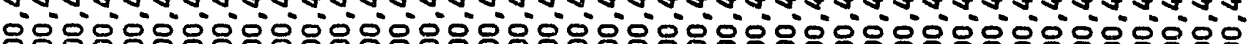
잉ㅇㅇㅇㅇㅇㅇㅇㅇㅇㅇㅇㅇㅇㅇㅇㅇㅇㅇㅇㅇㅇㅇㅇㅇㅇㅇㅇㅇㅇㅇㅇㅇㅇㅇㅇㅇㅇㅇㅇㅇㅇㅇㅇㅇㅇㅇㅇㅇㅇㅇㅇㅇㅇㅇㅇㅇㅇㅇㅇㅇㅇㅇ

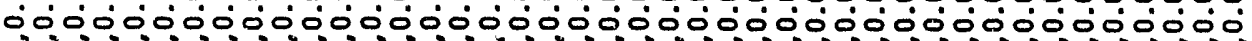

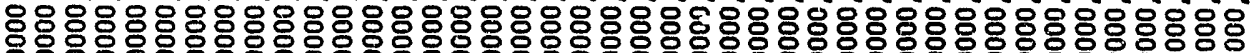

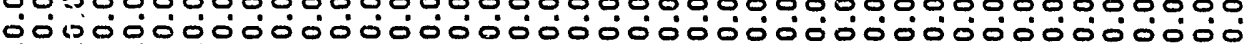

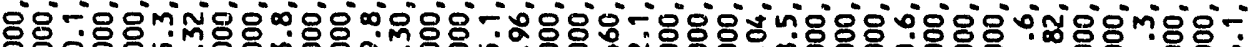

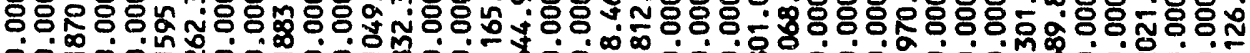
O $0^{\circ} 0^{\circ}$

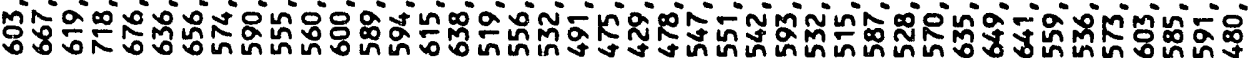
мm

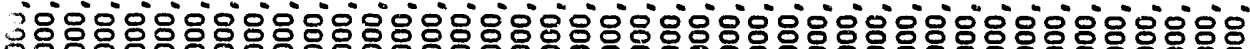

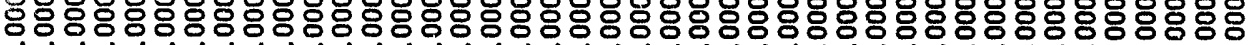

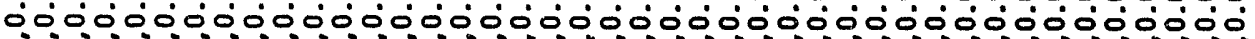

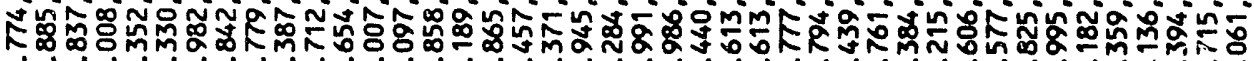

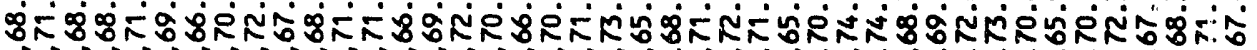
M० \%

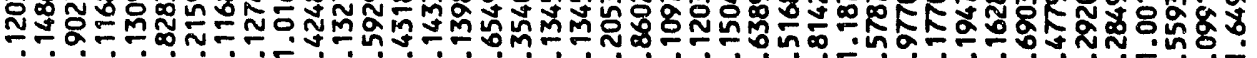

8і8

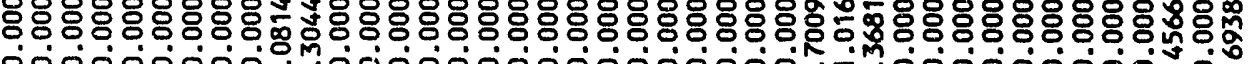
คำ

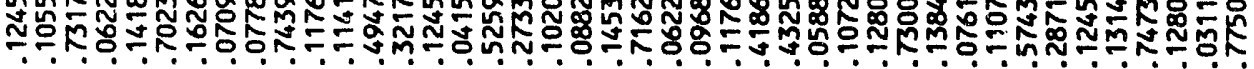

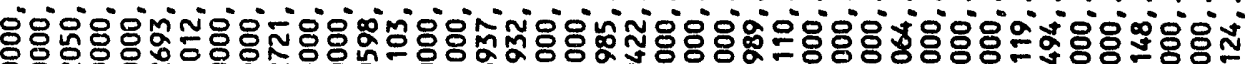

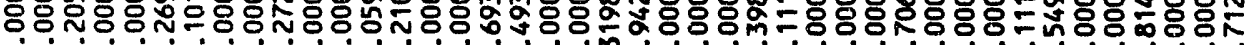

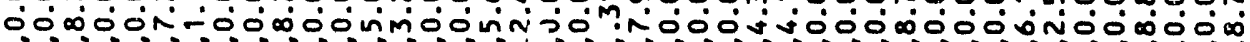

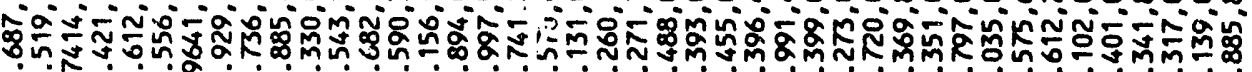

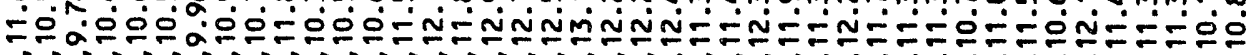

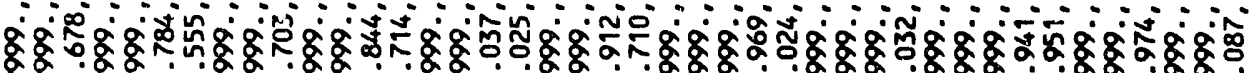

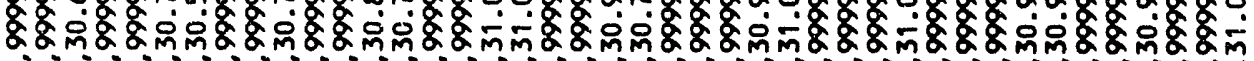

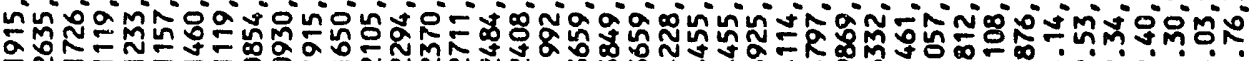

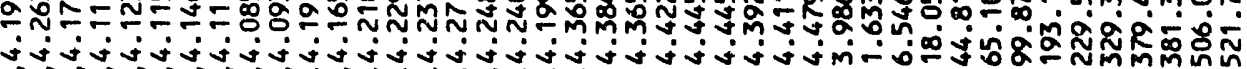
\&\%m

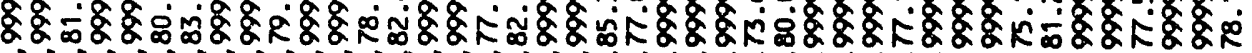

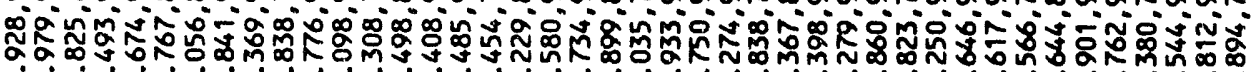
mร.

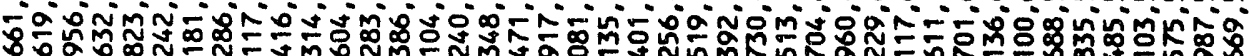

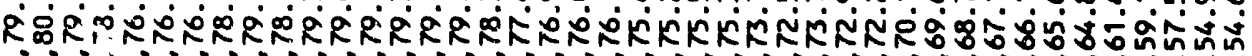
\& \&\& \&\%के

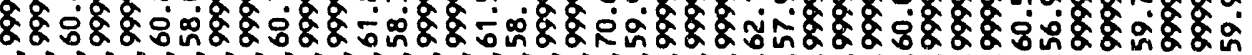

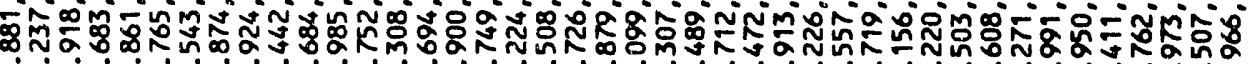

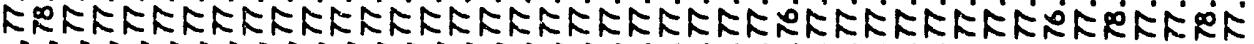
స్య

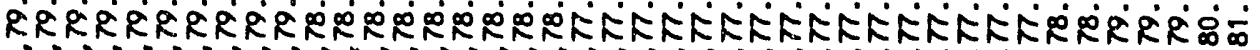

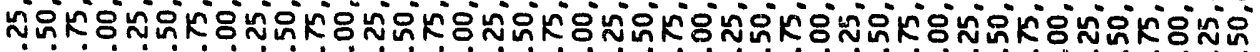

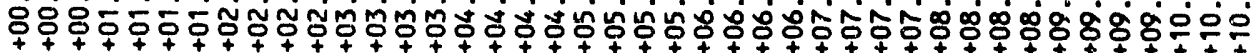

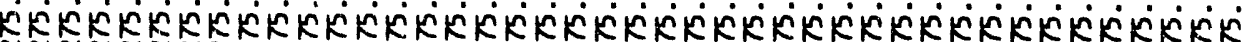

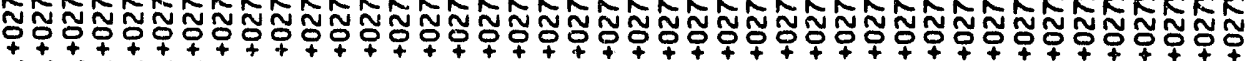

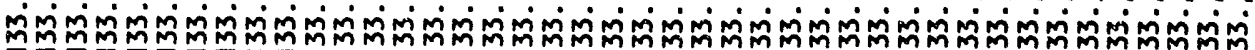

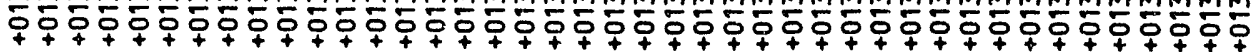




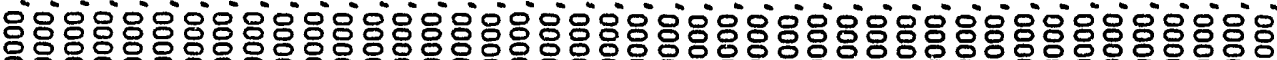

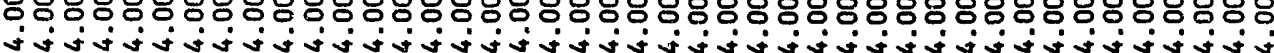

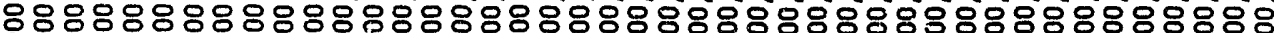

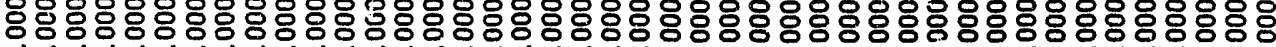

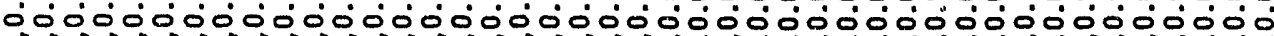

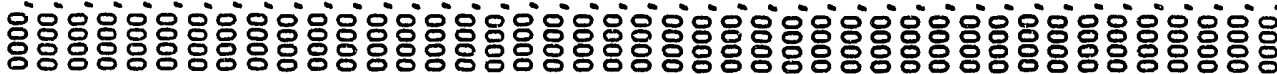
$00000000000000000000000000==5000000^{\circ} 0^{\circ} 0^{\circ} 0^{\circ} 0^{\circ}$

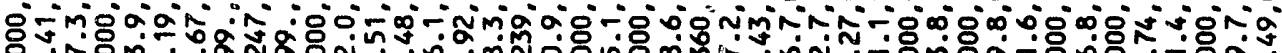

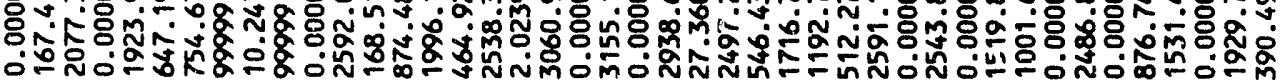
mo ro n.

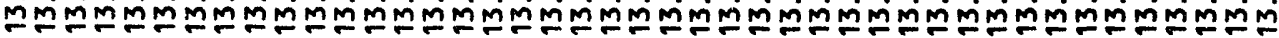

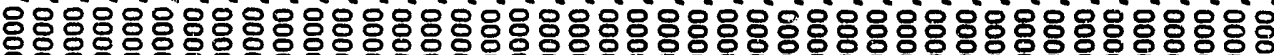

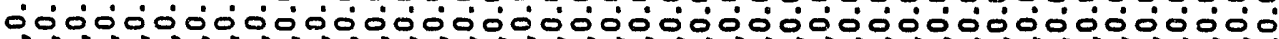

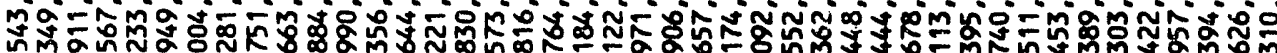

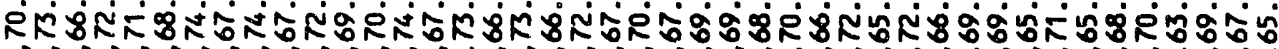

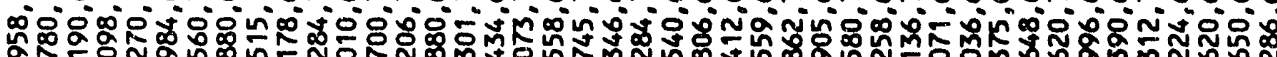

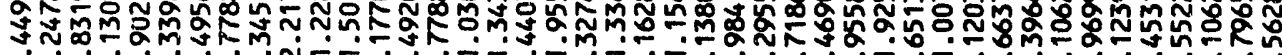

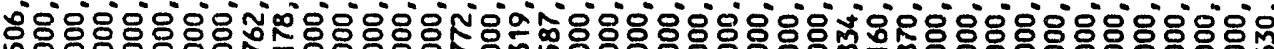

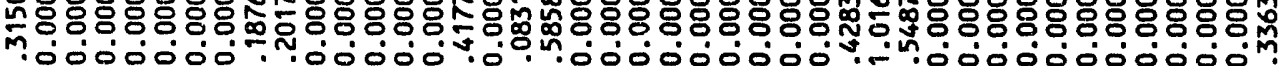
ผ 8: 8. sog รั) - jorvino şa ojojojojo

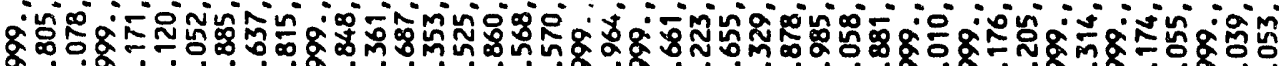
\%

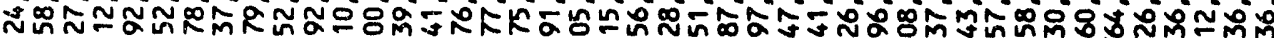

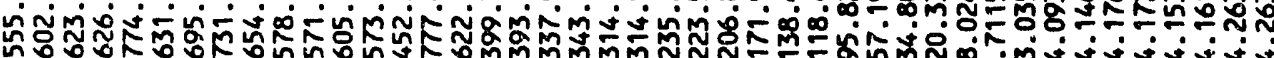

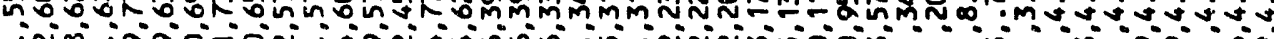
\& \& $F$ \%

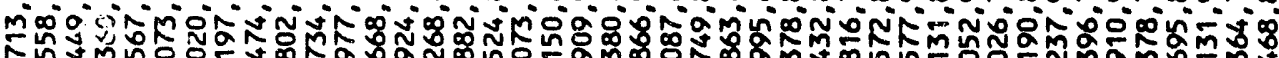
in

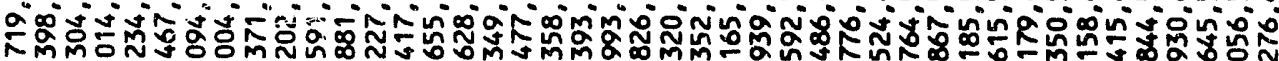

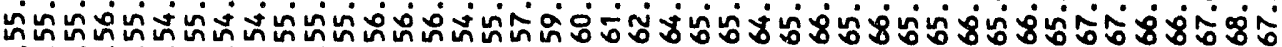

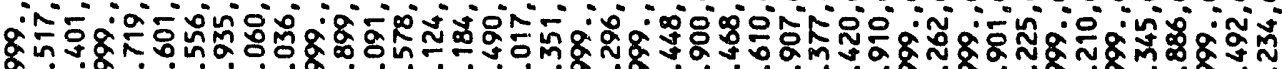

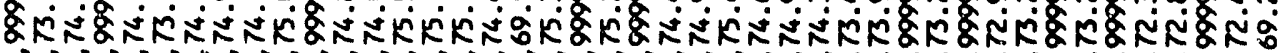
\% \& 8 \%

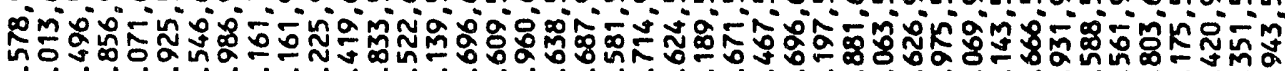

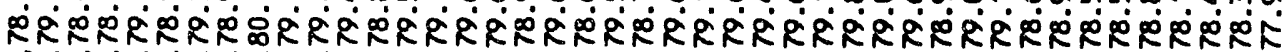

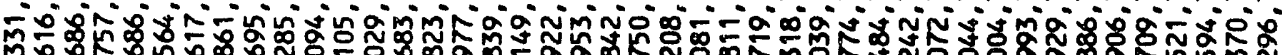
कळळ Kon

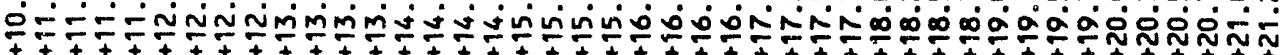

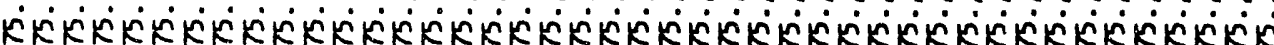

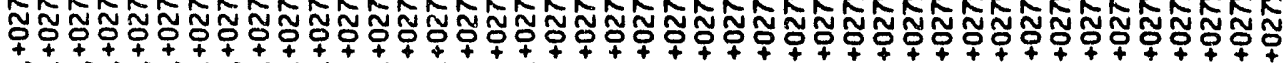

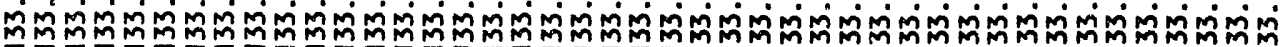

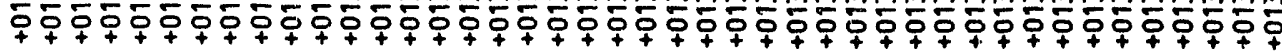




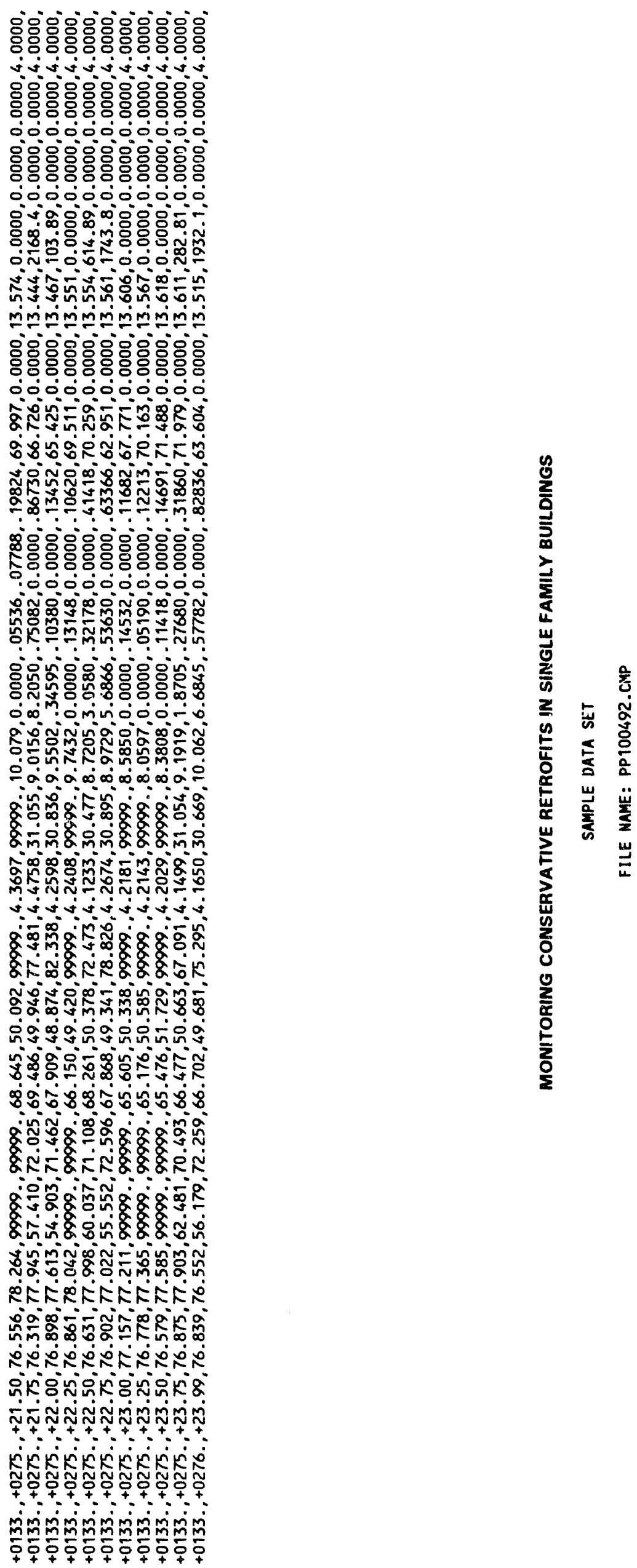




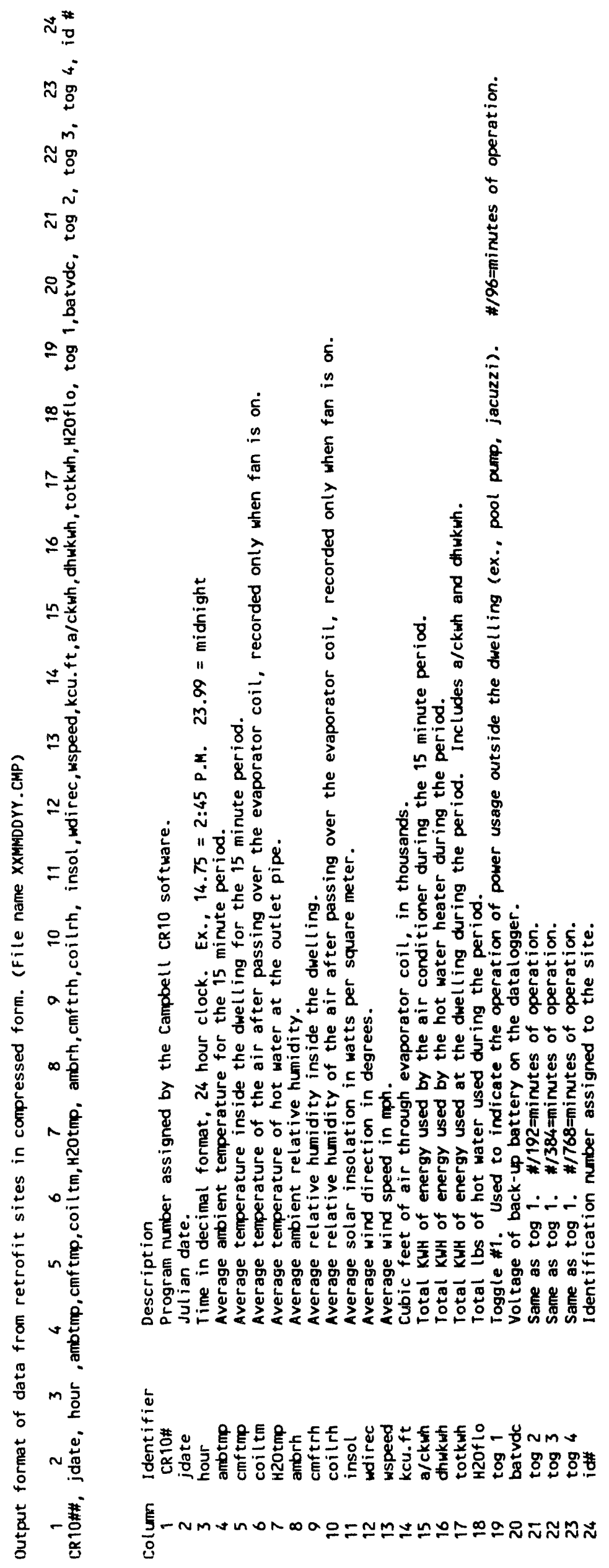


APPENDIX D

PRE-RETROFIT AUDIT DATA SHEETS

POST-RETROFIT AUDIT DATA SHEETS 


\section{PRE-RETROFIT AUDIT DATA FORM}

Retrofit program name Monitoring Conservative Retrofits in Single Family Bldgs Sponsoring organization(s) DOE/FPL/State of FL

Experimental site designation

Interviewer name CSR WL (非)

I. General information from householder

1. Name Wayne A. Legros

2. Street address 399 Charlotta Ave, S.E. City Palm Bay State FL 32909

3. Phone number (407)723-9069

4. Number of occupants, by age group:

\begin{tabular}{lc}
\multicolumn{1}{c}{ Age group } & Number \\
\hline Preschool & - \\
School age & -3 \\
Adult, but not retired & -2 \\
Retired & -
\end{tabular}

How many are generally home all day? Weekday Weekend

$6 / 3 / 91$

Date of interview

$6 / 3 / 91$ 
2. Style: One-story $\mathrm{x}$ Two-story Three-story Split-level One-and-a-half-story

3. Foundation: Basement Crawl space Slab Other (specify)

4. Exterior colors: Roof Dark Brown Walls Red brick

5. Layout, dimensions, orientativii. Use attached House Sketch Sheet.

6. Percentage of each floor that is conditioned (heated or cooled):

\begin{tabular}{|c|c|c|c|}
\hline Floor of house & $\begin{array}{c}\text { Percentage } \\
\text { heated }\end{array}$ & $\begin{array}{l}\text { Percentage } \\
\text { cooled }\end{array}$ & NA \\
\hline \multicolumn{4}{|l|}{ Exaseraxaxt } \\
\hline First floor & $100 \%$ & $100 \%$ & \\
\hline \multicolumn{4}{|l|}{ Secosodx Plosiox } \\
\hline XXhix & & & \\
\hline 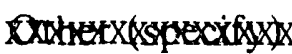 & & & \\
\hline
\end{tabular}

7. Infiltration: Use attached Infiltration Rate Evaluation Factors sheet.

8. Rooms typically closed off None

III. Major appliances

1. Domestic hot water

Type: Elec. resistance $\underline{\mathrm{X}}$ Heat pump hot water

Gas Solar

Other (specify)

Nameplate information, as available:

Manufacturer Ruud

Model PE 40-2

Tank capacity (gallons) 40 gal 4500 watt

SEER

Tank insulation ( $\mathrm{R}$-value)

Location Garage

Hot water temperature (measured with thermometer) in data set ${ }^{\circ} \mathrm{F}$

2. Solar devices

None $\mathrm{X}$ Domestic hot water __ Swimming pool heater

Space heating __ Other (specify) 
3. Other major appliances, by fuel type:

\begin{tabular}{|c|c|c|c|}
\hline Appliance & None & Electric Gas & $\begin{array}{l}\text { Other } \\
\text { (specify) }\end{array}$ \\
\hline Cooking range/oven & & $\mathrm{X}$ & \\
\hline Clothes washer & & $\mathrm{X}$ & \\
\hline Clothes dryer & & $\mathrm{X}$ & \\
\hline Refrigerator/freezer & & $\mathrm{X}$ & \\
\hline Separate freezer & & $\mathrm{X}$ & \\
\hline Conventional oven & & $\mathrm{X}$ & \\
\hline Microwave oven & & $\mathrm{X}$ & \\
\hline Dishwasher & & $\mathrm{X}$ & \\
\hline \multicolumn{4}{|l|}{ Whole house fan } \\
\hline \multicolumn{4}{|l|}{ Attic fan } \\
\hline Water bed heater & & 3 & \\
\hline Other & & Water pump & \\
\hline
\end{tabular}

IV. Ceiling area and insulation

(If different parts of the ceiling have different insulation, treat each of these sub-areas separately.)

\begin{tabular}{cccc} 
Sub-area & $\begin{array}{c}\text { Area } \\
\left(\mathrm{ft}^{2}\right)\end{array}$ & $\begin{array}{c}\text { Insulation } \\
(\mathrm{R})\end{array}$ & $\begin{array}{c}\text { Insulation } \\
\text { type }\end{array}$ \\
\hline $\begin{array}{c}\text { 1. } \\
2 .\end{array}$ & 1248 & 19 & blown \\
3. & & & \\
Total & 1248 $\left(\mathrm{ft}^{2}\right)$ & &
\end{tabular}

V. Wall area and insulation

(If different parts of wall have different materials, treat each of these sub-areas separately.)

Exterior wall construction: (1)Wood; (2) Brick; (3) Stone; (4) Stucco;

(5) Concrete block; (6) Metal;

(7) Other (specify) drywal1 between garage

\begin{tabular}{ccccc} 
Sub-area & $\begin{array}{c}\text { Area } \\
\left(\mathrm{ft}^{2}\right)\end{array}$ & $\begin{array}{c}\text { Insulation } \\
(\mathrm{R})\end{array}$ & $\begin{array}{c}\text { Exterior wall } \\
\text { construction }\end{array}$ & $\begin{array}{c}\text { Insulation } \\
\text { type }\end{array}$ \\
\hline 1. 935.9 & 11 & brick & batts \\
2. & 261.5 & 11 & drywall & batts (garage) \\
3. & & & & \\
Total $1197.4\left(\mathrm{ft}^{2}\right)$ & & &
\end{tabular}


VI. Floor area and insulation (bottom floor only)

(If different parts of floor have different materials, treat each of these sub-areas separately.)

\begin{tabular}{cccccc} 
Sub-area & $\begin{array}{c}\text { Area } \\
\left(\mathrm{ft}^{2}\right)\end{array}$ & $\begin{array}{c}\text { Insulation } \\
(\mathrm{R})\end{array}$ & $\begin{array}{c}\text { Percent } \\
\text { carpeted }\end{array}$ & $\begin{array}{c}\text { What is below? } \\
\text { (slab, crawl space, } \\
\text { basement?) }\end{array}$ & $\begin{array}{c}\text { Insulation } \\
\text { type }\end{array}$ \\
\hline $\begin{array}{c}1 . \\
2 .\end{array}$ & 1248 & - & $68 \%$ & slab & none \\
$\begin{array}{c}\text { 3. } \\
\text { Total }\end{array}$ & & & & \\
\end{tabular}

Vil. Windows and glass doors

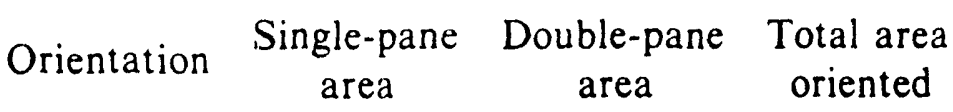

\begin{tabular}{lll}
$\begin{array}{l}\text { Front of } \\
\text { house }\end{array}$ & $46 \mathrm{sq} \mathrm{ft} \quad 46 \mathrm{sq} \mathrm{ft}$ \\
\hline $\begin{array}{l}\text { Left side } \\
\text { of house }\end{array}$ & none \\
\hline $\begin{array}{l}\text { Right side } \\
\text { of house }\end{array}$ & $4 \mathrm{sq} \mathrm{ft} \quad 4 \mathrm{sq} \mathrm{ft}$
\end{tabular}

\begin{tabular}{lll}
$\begin{array}{l}\text { Back of } \\
\text { house }\end{array}$ & $80 \mathrm{sq} f t$ & $80 \mathrm{sq} \mathrm{ft}$ \\
+ & \\
\hline
\end{tabular}

VIIl. Doors (non-glass)

\begin{tabular}{|c|c|c|c|}
\hline Orientation & $\begin{array}{c}\text { Regular } \\
\text { door } \\
\text { area }\end{array}$ & $\begin{array}{l}\text { Regular door with } \\
\text { storm door or } \\
\text { thermally insulated } \\
\text { door area }\end{array}$ & $\begin{array}{c}\text { Total } \\
\text { area }\end{array}$ \\
\hline Front of house & -- & 19.5 & $19.5 \mathrm{sq} \mathrm{ft}$ \\
\hline Left side of house & -- & - & \\
\hline Right side of house & 17.3 & -- & $17.3 \mathrm{sq}$ \\
\hline Back of house & -- & -- & \\
\hline Totals & 17.3 & 19.5 & $36.8 \mathrm{sq} \mathrm{ft}$ \\
\hline
\end{tabular}


IX. Heating and cooling systems

1. Main heating system type:

Gas furnace Gas boiler (steam)

Gas boiler (hot water) Oil furnace

Oil boiler (steam) Oil boiler (hot water)

Elec. furnace Elec. ceiling Elec. baseboard

Central heat pump Window heat pump

Other (specify) Resistance coils

If oil fired, has a flame retention head burner been installed?

Yes No

If oil boiler, is it wet base or dry base

Approximate age of system 8 years

Location of unit within house in $\mathrm{A} / \mathrm{H}$ unit in garage

Is this a converted coal unit? Yes No

Heating value of fuel: Gas Oil

Nameplate information, as available:

Manufacturer Singer

Model BCE 22008-30-11

Innut capacity Btu/hr

Output capacity $\mathrm{Btu} / \mathrm{hr}$

SEER

Is the pilot light turned off in the spring? Yes No $\mathrm{N} / \mathrm{A} \underline{\mathrm{X}}$ Energy efficiency devices present:

Vent damper IID Other (specify)

2. Distribution system

No ductwork or piping Hydronic (pumped) Hydronic (gravity)

Baseboard Forced-air $x$

Other (specify) 
If ductwork or piping present:

Total length of ducts or piping $94 \mathrm{ft}$

Percentage of ducts or piping in unconditioned (non-living) spaces $100 \%$ Are ducts or piping in unconditioned spaces insulated?

Yes $\mathrm{X}$ No __ If yes, approximate R-value

3. Main cooling system type:

None Window $\mathrm{A} / \mathrm{C}$ Central A/C $\mathrm{x}$

Evaporative cooler Central heat pump

Window heat pump Other (specify)

Approximate age of system 8 years

Nameplate information, as available

Manufacturer Singer

Model ${ }_{A C}^{B C E} 50029-15 F^{2}-11$ air handler

Input capacity $\mathrm{Btu} / \mathrm{hr}$

Output capacity _ But $/ \mathrm{hr}$

SEER Unknown

Location(s) within house $\mathrm{A} / \mathrm{H}$ in garage

4. Secondary heating systems:

No. fireplaces None

No. fireplace inserts

No. wood stoves

No. electric space heaters -

No. fossil fuel space heaters

Other (specify)

How often are these used?

5. Thermostat:

None Normal $x$

Clock Other (specify)

Location within house On south wall of living room above $A / H$ return 
X. Significant external loads:

Irrigation pumps

Welder

Outside lights Other (specify) pool pump \& well pump

Frequency of use Pool pump approximately $3 \mathrm{hrs}$ daily (owner's estimate)

XI. Experimental retrofit installation:

Description Lennox ECB 19-10.0-1P A/H

Lennox HS 22-31 1V-1P compressor

Heat Recovery: ECU Hot Tap D506

Actual installation cost $\$$

$\mathrm{A} / \mathrm{C}: \quad 2800.00$

Heat Recovery: $\quad 425.00$ 


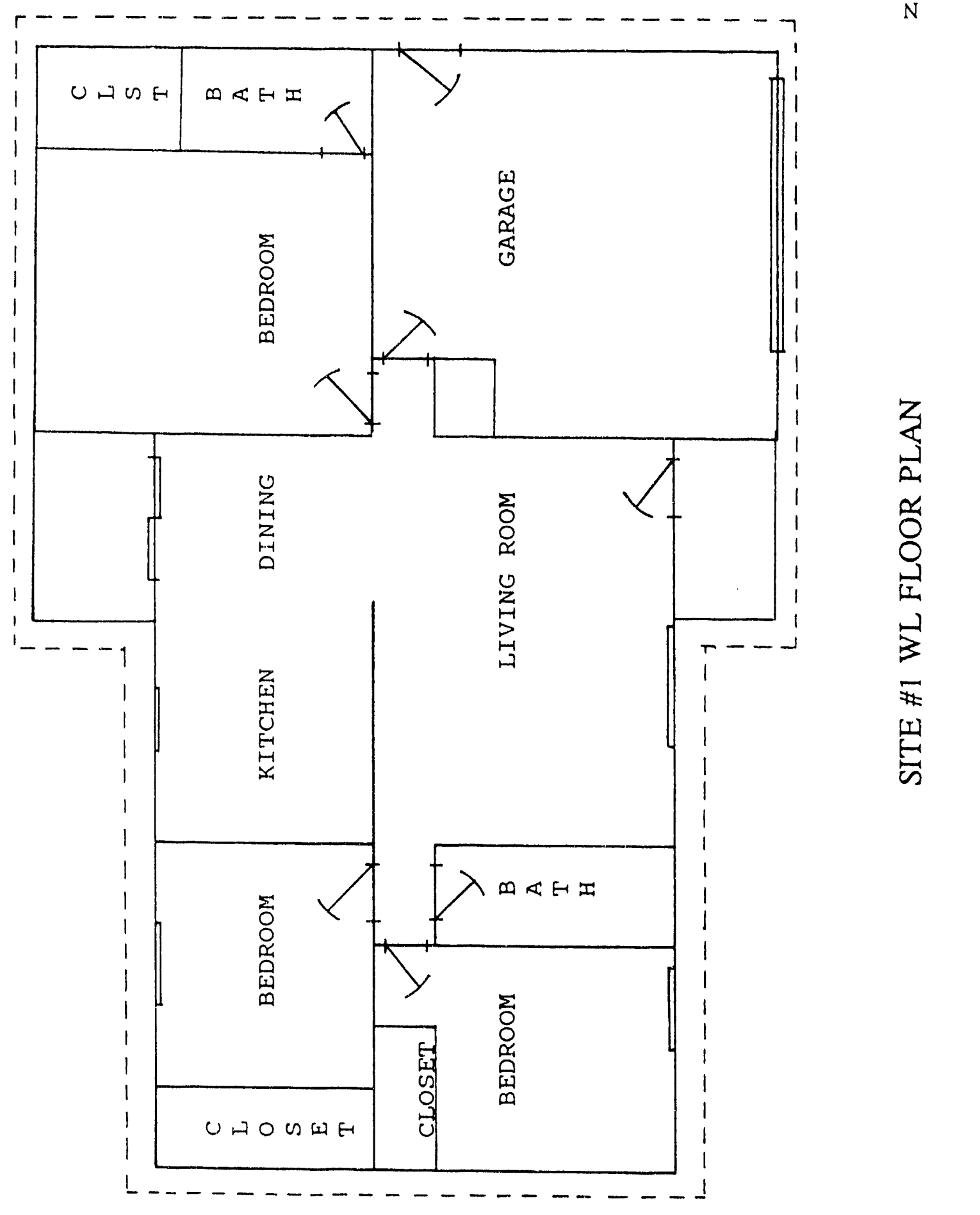



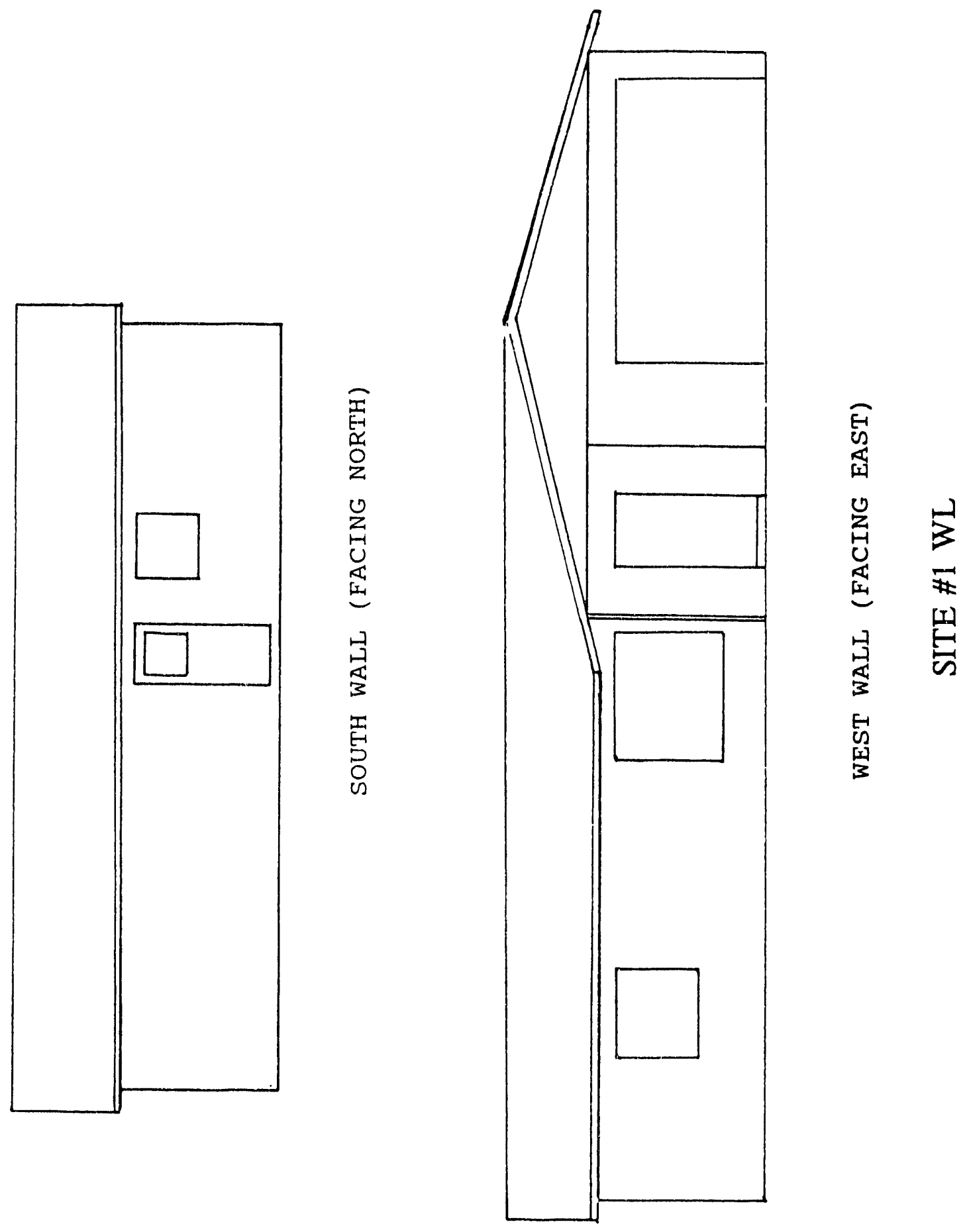

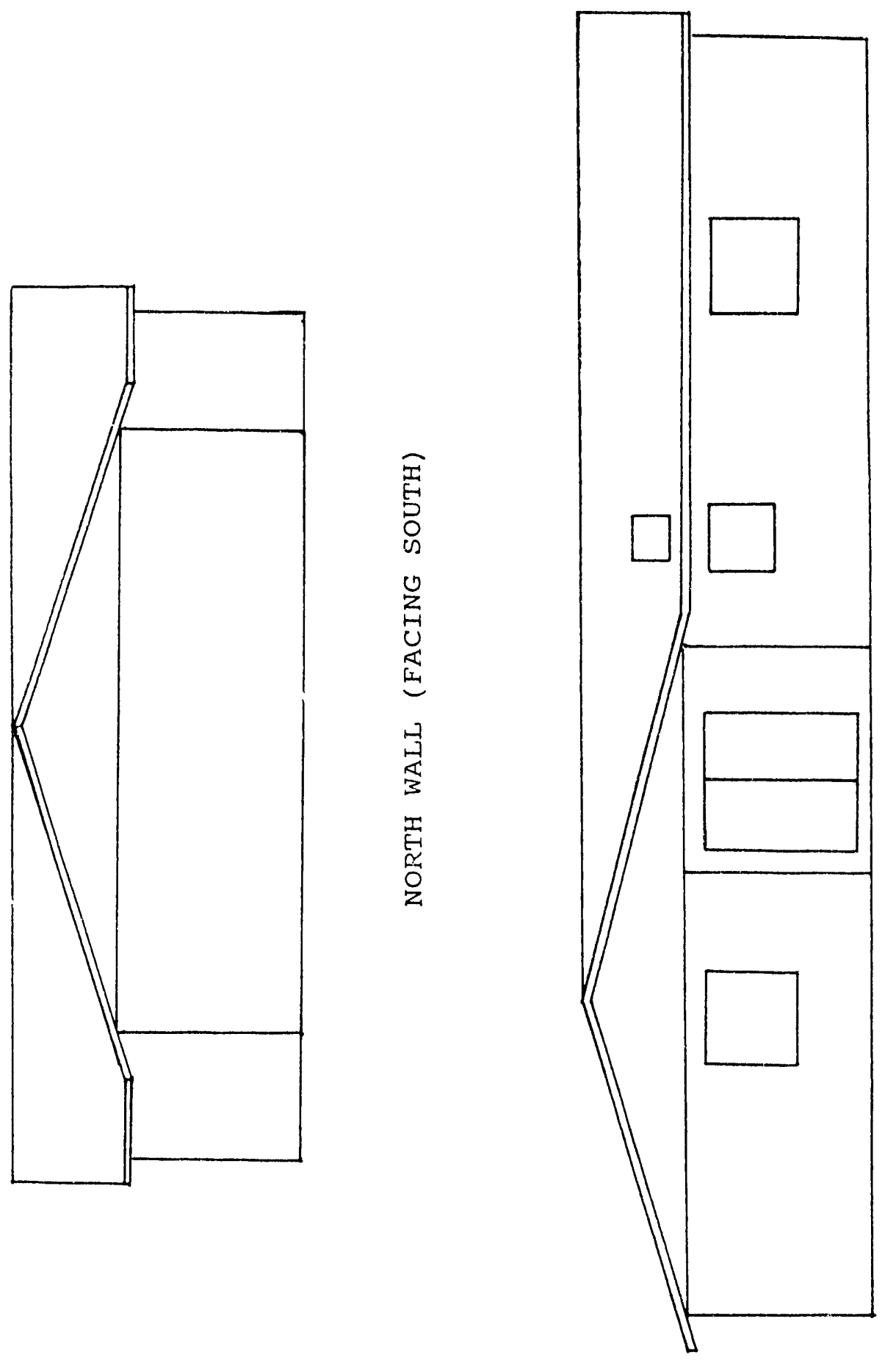

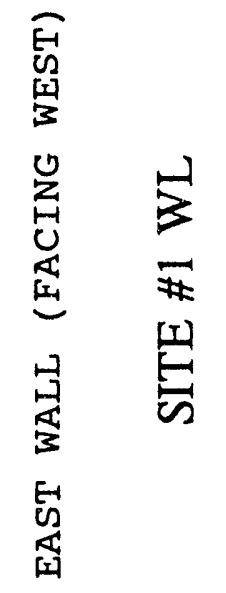


INFILTRATION RATE EVALUATION FACTORS

\begin{tabular}{|c|c|c|c|}
\hline $\begin{array}{l}\text { Building } \\
\text { component }\end{array}$ & Good & Average & Poor \\
\hline $\begin{array}{l}\text { Windows } \\
\text { and } \\
\text { doors }\end{array}$ & $\begin{array}{l}\text { X Window and door frames } \\
\text { caulked. Window and door } \\
\text { sashes well fitting and weather- } \\
\text { stripped or storm windows and } \\
\text { doors with good fit. }\end{array}$ & $\begin{array}{l}\text { Window and door frames } \\
\text { caulked or window and door } \\
\text { sashes weatherstripped or } \\
\text { poorly fitting storm doors and } \\
\text { windows. }\end{array}$ & $\begin{array}{l}\text { No caulking on window and } \\
\text { door frames. No weather- } \\
\text { stripping. No storm doors or } \\
\text { windows. }\end{array}$ \\
\hline $\begin{array}{l}\text { Walls and } \\
\text { electrical } \\
\text { outlets }\end{array}$ & $\begin{array}{l}\text { Xeiling and noor joints and } \\
\text { corners well sealed. Elec- } \\
\text { trical outlets with gaskets. No } \\
\text { holes around plumbing penetra- } \\
\text { tions. }\end{array}$ & $\begin{array}{l}\text { Some cracks in ceiling and } \\
\text { floor joints and corners. No } \\
\text { gaskets on electrical outlets. } \\
\text { Fewer than three plumbing } \\
\text { penetrations with holes } \\
\text { around them. }\end{array}$ & $\begin{array}{l}\text { Many cracks in ceiling, } \\
\text { floor joints, and corners. No } \\
\text { gaskets on electrical outlets. } \\
\text { Three or more plumbing } \\
\text { penetrations with holes } \\
\text { around them. }\end{array}$ \\
\hline $\begin{array}{l}\text { Attic } \\
\text { floor } \\
\text { (ceiling) }\end{array}$ & $\begin{array}{l}\text { X No cracks in attic floor. No } \\
\text { air shafts around flues. No } \\
\text { holes around ducts, pipes, or } \\
\text { wiring penetrating attic floor. } \\
\text { No recessed light fixtures. No } \\
\text { trap door or weatherstripped } \\
\text { trap door to attic. }\end{array}$ & $\begin{array}{l}\text { Some cracks in attic foor. } \\
\text { No air shafts around flues. } \\
\text { Some holes around ducts, pipes, } \\
\text { or wiring penetrating attic floor. } \\
\text { Fewer than three recessed light } \\
\text { fixtures. Unweatherstripped } \\
\text { trap door to attic. }\end{array}$ & $\begin{array}{l}\text { Many cracks in attic floor. } \\
\text { Air shafts around flues. } \\
\text { Holes around ducts, pipes, or } \\
\text { wiring penetrating attic floor. } \\
\text { More than three recessed light } \\
\text { fixtures. Uncovered attic } \\
\text { access. }\end{array}$ \\
\hline $\begin{array}{l}\text { Heating } \\
\text { system } \\
\text { and water } \\
\text { heater }\end{array}$ & $\begin{array}{l}\text { Xoth furnace and water } \\
\text { heater electric. If fossil fuel } \\
\text { fired. both in unconditioned } \\
\text { space. }\end{array}$ & $\begin{array}{l}\text { One fossil fuel-fired unit in } \\
\text { living space with vent } \\
\text { damper. The other in uncondi- } \\
\text { tioned space. }\end{array}$ & $\begin{array}{l}\text { At least one fossil fuel-fired } \\
\text { unit in living space without } \\
\text { vent damper. }\end{array}$ \\
\hline $\begin{array}{l}\text { Fireplace } \\
\text { or Hood } \\
\text { stove }\end{array}$ & $\begin{array}{l}\text { Sealed cornbustion wood } \\
\text { stove or fireplace with well- } \\
\text { fitting damper and glass doors } \\
\text { or no fireplace. }\end{array}$ & $\begin{array}{l}\square \text { Poorly sealed wood stove } \\
\text { or fireplace with either well- } \\
\text { fitting damper or glass doors. }\end{array}$ & $\begin{array}{l}\text { Both a wood stove and fire- } \\
\text { place or a fireplace with } \\
\text { poorly fitting damper and no } \\
\text { glass doors. }\end{array}$ \\
\hline $\begin{array}{l}\text { Ductwork } \\
\text { and } \\
\text { noor }\end{array}$ & $\begin{array}{l}7 \text { No ductwork and few floor } \\
\text { penetrations or all ductwork } \\
\text { in conditioned space and } \\
\text { no floor penetration. }\end{array}$ & $\begin{array}{l}\text { Ductwork in conditioned } \\
\text { basement and few floor } \\
\text { penetrations. }\end{array}$ & 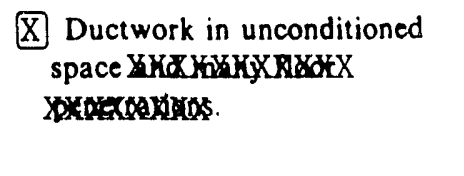 \\
\hline $\begin{array}{l}\text { Vents in } \\
\text { conditioned } \\
\text { space }\end{array}$ & $\begin{array}{l}\text { No undampered vents and } \\
\text { fewer than three dampered } \\
\text { vents. }\end{array}$ & $\begin{array}{l}\text { Fewer than three undampered } \\
\text { vents or at least three } \\
\text { dampered vents. }\end{array}$ & $\begin{array}{l}\square \text { More than three undampered } \\
\text { vents. }\end{array}$ \\
\hline Lifestyle & $\begin{array}{l}\square \text { Fewer than six entrances and } \\
\text { exits per day. }\end{array}$ & $\begin{array}{l}\text { Six to thirteen entrances } \\
\text { and exits per day. }\end{array}$ & $\begin{array}{l}\text { More than thirtcen } \\
\text { entrances and exits per day. }\end{array}$ \\
\hline
\end{tabular}




\section{POST-RETROFIT AUDIT DATA FORM}

Retrofit program name Monitoring Conservative Retrofits in Single Family Bldgs

Sponsoring organization(s) DOE/FPL/State of FL

Experimental site designation

Interviewer name CSR

Date of interview

$11 / 22 / 92$

1. General information from householder

1. Name Wayne A. Legros

2. Street address

City State

3. Phone number

4. Ow'n Rent

5. If householder different from that of "Pre-Retrofit Audit," or if tenure has changed, record below:

Change in householder Date of change

Change in tenure Date of change

6. Number of occupants, by age group:

\begin{tabular}{ll}
\multicolumn{1}{c}{ Age group } & Number \\
\hline Preschool & \\
School age & 3 \\
Adult, but not retired & 2 \\
Retired &
\end{tabular}

How many are generally home all day?

\begin{tabular}{ll} 
Weekday & 3 \\
Weekend & 5 \\
\hline
\end{tabular}

II. Significant changes since pre-retrofit audit NONE

(The remaining questions refer to the time since the energy-saving improvements were made to the house).

1. Have there been any important changes or additions to the house, such as (check appropriate): No

Rooms added Siding added

Garage/carport added Exterior paint 
Porch added

New roof

Other (specify)

2. Other than changes of this program, has the main heating or cooling system been changed, such as (check appropriate):

New furnace/boiler No

New air conditioner No

New heat pump _ No

Modified furnace/boiler (specify) No

Other (specify) No

3. Are the same number of rooms still heated and cooled? (If not, specify) yes

4. Have any space heaters been added/subtracted? (Fireplaces, wood stoves, electric, fossil fuel, etc.) Yes No $\mathrm{X}$

If so, specify

Has the use of these heaters changed during this last winter?

Used more this winter __ Less _ Same $\mathrm{X}$ - Do not know _-

5. Has the hot water heating system changed? Yes _ No -

If so, specify

6. Have any major appliances been added/subtracted? (Stoves, ovens, washers, dryers, etc.)

If so, specify No

7. Is there: An attic fan No No Whole house fan No

If so, has the use of these fans changed during this last summer?

Used more this summer __ Less _ Same __ Do not know

8. Other than changes of this program, have any other energy-saving cinanges been installed this past year? Yes __ No $\mathrm{X}$

If yes, specify:

Ceiling insulation added Weatherstripping

Wall insulation added Caulking

Storm windows added Hot water flow restrictors

Storm doors added Clock thermostat

Other (specify) 
9. Have you or any family members taken up any new hobbies during the past year that require the use of large electrical or gas equipment?

Yes

If so, specify 


\section{PRE-RETROFIT AUDIT DATA FORM}

Retrofit program name Monitoring Conservative Retrofits in S.F.B

Sponsoring organization(s) DOE/FPL/State of FL

Experimental site designation $\mathrm{RH}$ (非2)

Interviewer name Date of interview $\quad$ CSR

1. General information from householder

1. Name Rose Hirt

2. Street address 2817 Kensington Drive

City Melbourne

State

FL

3. Phone number

$255-0279$

4. Number of occupants, by age group:

\begin{tabular}{ll}
\multicolumn{1}{c}{ Age group } & Number \\
\hline Preschool & \\
School age & \\
Adult, but not retired & 3 \\
Retired &
\end{tabular}

How many are generally home all day? Weekday

Weekend 3-4

5. Approximate age of house 13 years

6. Names of utility distributors:

Electric FPL

Gas

Oil

(Note: Auditor should have homeowner sign a release authorizing release by these utilities of fuel bills for preceding two years.)

7. Ow'n Rent

8. Length of time at this address 13 years

II. General description of house

1. Type: S-F detached $-\mathrm{X}$ S-F attached

Mobile home

Other (specify) 
2. Style: One-story $\mathrm{x} \quad$ Two-story _ Three-story Split-level __ One-and-a-half-story

3. Foundation: Basement Crawl space Slab $\underline{x}$ Other (specify)

4. Exterior colors: Roof beige Walls tan stucce

5. Layout, dimensions, orientation: Use attached House Sketch Sheet.

6. Percentage of each floor that is conditioned (heated or cooled):

\begin{tabular}{lccc} 
Floor of house & $\begin{array}{c}\text { Percentage } \\
\text { heated }\end{array}$ & $\begin{array}{c}\text { Percentage } \\
\text { cooled }\end{array}$ & NA \\
\hline $\begin{array}{l}\text { Basement } \\
\text { First floor }\end{array}$ & $100 \%$ & $100 \%$ & $\mathrm{X}$ \\
$\begin{array}{l}\text { Second floor } \\
\text { Third floor }\end{array}$ & & & \\
Other (specify) & & &
\end{tabular}

7. Infiltration: Use attached Infiltration Rate Evaluation Factors sheet.

8. Rooms typically closed off None

III. Major appliances

1. Domestic hot water

Type: Elec. resistance $\mathrm{X}$ Heat pump hot water

Gas Solar

Other (specify)

Nameplate information, as available:

Manufacturer Smith Permaglas

Model EEST 40

Tank capacity (gallons)

SEER

Tank insulation ( $R$-value)

Location Laundry Room

Hot water temperature (measured with thermometer) 120 ${ }^{\circ} \mathrm{F}$

2. Solar devices

None $x \quad$ Domestic hot water Swimming pool heater

Space heating Other (specify) 
3. Other major appliances, by fuel type:

\begin{tabular}{|c|c|c|c|c|c|}
\hline Appliance & None & Electric & Gas & $\begin{array}{l}\text { Oth:r } \\
\text { (specify) }\end{array}$ & Location \\
\hline Cooking range/oven & & $\mathrm{x}$ & & & \\
\hline Clothes washer & & $\mathrm{x}$ & & & \\
\hline Clothes dryer & & $\mathrm{X}$ & & & \\
\hline $\begin{array}{l}\text { Refrigerator/freezer } \\
\text { Separate freezer }\end{array}$ & & $\mathrm{X}$ & & & \\
\hline Conventional oven & & $\mathrm{x}$ & & & \\
\hline Microwave oven & & $\mathrm{X}$ & & & \\
\hline Dishwasher & & $\mathrm{x}$ & & & \\
\hline Whole house fan & & & & & \\
\hline Attic fan & & & & & \\
\hline Water bed heater & & & & & \\
\hline Other & & & & & \\
\hline
\end{tabular}

IV. Ceiling area and insulation

(If different parts of the ceiling have different insulation, treat each of these sub-areas separately.)

\begin{tabular}{cccc} 
Sub-area & $\begin{array}{c}\text { Area } \\
\left(\mathrm{ft}^{2}\right)\end{array}$ & $\begin{array}{c}\text { Insulation } \\
(\mathrm{R})\end{array}$ & $\begin{array}{c}\text { Insulation } \\
\text { type }\end{array}$ \\
\hline $\begin{array}{c}\text { 1. } \\
2 .\end{array}$ & 1257 & $<7$ & batts \\
3 & & & \\
Total & & 1257 & $\left(\mathrm{ft}^{2}\right)$
\end{tabular}

V. Wall area and insulation

(If different parts of wall have different materials, treat each of these sub-areas separately.)

Exterior wall construction: (1)Wood; (2) Brick; (3) Stone; (4) Stucco;

(5) Concrete block; (6) Metal;

(7) Other (specify)

\begin{tabular}{ccccc} 
Sub-area & $\begin{array}{c}\text { Area } \\
\left(\mathrm{ft}^{2}\right)\end{array}$ & $\begin{array}{c}\text { Insulation } \\
(\mathrm{R})\end{array}$ & $\begin{array}{c}\text { Exterior wall } \\
\text { construction }\end{array}$ & $\begin{array}{c}\text { Insulation } \\
\text { type }\end{array}$ \\
\hline $\begin{array}{c}1 . \\
2 .\end{array}$ & 1312 & unk & block \\
$\begin{array}{c}\text { 3. } \\
\text { Total }\end{array}$ & & & & \\
\end{tabular}


VI. Floor area and insulation (bottom floor only)

(If different parts of floor have different materials, treat each of these sub-areas separately.)

\begin{tabular}{lccccc} 
Sub-area & $\begin{array}{c}\text { Area } \\
\left(\mathrm{ft}^{2}\right)\end{array}$ & $\begin{array}{c}\text { Insulation } \\
(\mathrm{R})\end{array}$ & $\begin{array}{c}\text { Percent } \\
\text { carpeted }\end{array}$ & $\begin{array}{c}\text { What is below? } \\
\text { (slab, crawl space, } \\
\text { basement?) }\end{array}$ & $\begin{array}{c}\text { Insulation } \\
\text { type }\end{array}$ \\
\hline $\begin{array}{l}\text { 1. } \\
\text { 2. }\end{array}$ & 1257 & - & $36 \%$ & Slab & -- \\
3. & & & & & \\
Total & $1257\left(\mathrm{ft}^{2}\right)$
\end{tabular}

VII. Windows and glass doors

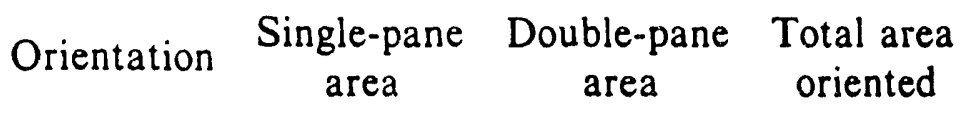

\begin{tabular}{llll}
\hline $\begin{array}{l}\text { North } \\
\text { Front of } \\
\text { house }\end{array}$ & 27.68 & 24.00 & 51.68 \\
\hline $\begin{array}{l}\text { East } \\
\text { Left side } \\
\text { of house }\end{array}$ & 33.33 & 46.67 & 80.00 \\
\hline $\begin{array}{l}\text { West } \\
\text { Right side } \\
\text { of house }\end{array}$ & 16.0 & & \\
$\begin{array}{l}\text { South } \\
\text { Back of } \\
\text { house }\end{array}$ & 22.29 & 37.5 & \\
& & & \\
\end{tabular}

VIII. Doors (non-glass)

\begin{tabular}{lccc} 
Orientation & $\begin{array}{c}\text { Regular } \\
\text { door } \\
\text { area }\end{array}$ & $\begin{array}{c}\text { Regular door with } \\
\text { storm door or } \\
\text { thermally insulated } \\
\text { door area }\end{array}$ & $\begin{array}{c}\text { Total } \\
\text { area }\end{array}$ \\
\hline $\begin{array}{l}\text { Front of house } \\
\text { Left side of house } \\
\text { West side of house }\end{array}$ & 17.33 & & 17.33 \\
$\begin{array}{l}\text { Back of house } \\
\text { Totals }\end{array}$ & 17.33 & & 17.33 \\
\hline
\end{tabular}


IX. Heating and cooling systems

1. Main heating system type:

Gas furnace

Gas boiler (steam)

Gas boiler (hot water)

Oil furnace

Oil boiler (steam) Oil boiler (hot water)

Elec. furnace Elec. ceiling Elec. baseboard

Central heat pump Other (specify) Resistance coils in $\mathrm{A} / \mathrm{H}$

If oil fired, has a flame retention head burner been installed?

Yes No

If oil boiler, is it wet base or dry base

Approximate age of system years

Location of unit within house

Is this a converted coal unit? Yes No

Heating value of fuel: Gas

Oil

Nameplate information, as available:

Manufacturer See $A / C$

Modei

Input capacity Btu/hr

Output capacity $\mathrm{Btu} / \mathrm{hr}$

SEER

Is the pilot light turned off in the spring? Yes No N/A $\underline{X}$ Energy efficiency devices present:

Vent damper IID Other (specify)

2. Distribution system

No ductwork or pipin, Hydronic (pumped)

Baseboard Hydronic (gravity) Forced-air $\mathrm{X}$

Other (specify) 
If ductwork or piping present:

Total length of ducts or piping 115

Percentage of ducts or piping in unconditioned (non-living) spaces $100 \%$

Are ducts or piping in unconditioned spaces insulated?

Yes $\mathrm{X}$ No __ If yes, approximate R-value 5

3. Main cooling system type:

None Window A/C Central A/C before

Evaporative cooler Central heat pump

Window heat pump Other (specify)

Approximate age of system 13

Nameplate information, as available.

Manufacturer Singer (BCE 22008-29-11 AH)

Model AC 50029-15F comp. unit

Input capacity $\mathrm{Btu} / \mathrm{hr}$

Output capacity $\mathrm{But} / \mathrm{hr}$

SEER

Location(s) within house At closet, east side of house

4. Secondary heating systems:

No. fireplaces None

No. fireplace inserts None

No. wood stoves None

No. electric space heaters №ne

No. fossil fuel space heaters None

Other (specify) None

How often are these used?

5. Thermostat:

None

Normal $-\mathrm{x}$

Clock Other (specify)

Location within house 
$X$. Significant external loads:

Irrigation pumps Welder

Outside lights Other (specify)

Frequency of use

XI. Experimental retrofit installation:

Description Lennox CB 18-41-3P \& Lennox HP-22-311-2P

Insulation upgrade to R19

ECU Hot Tap heat recovery unit

Actual installation cost $\$$
$\mathrm{A} / \mathrm{C}$
2925.00
( 327.00 rebate)
Heat Recovery
450.00
( 35.00 rebate)
Insulation
243.00
( 142.00 rebate) 

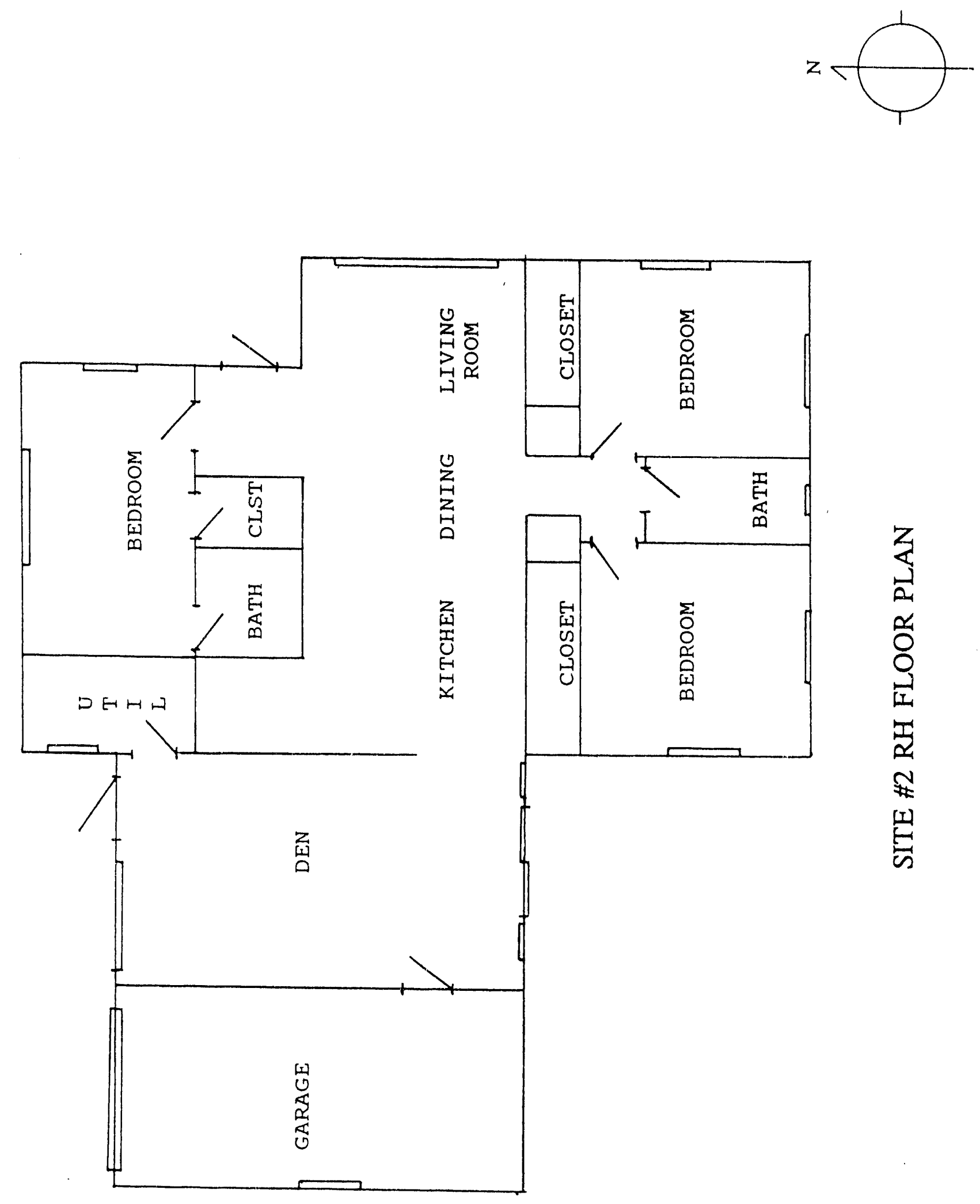

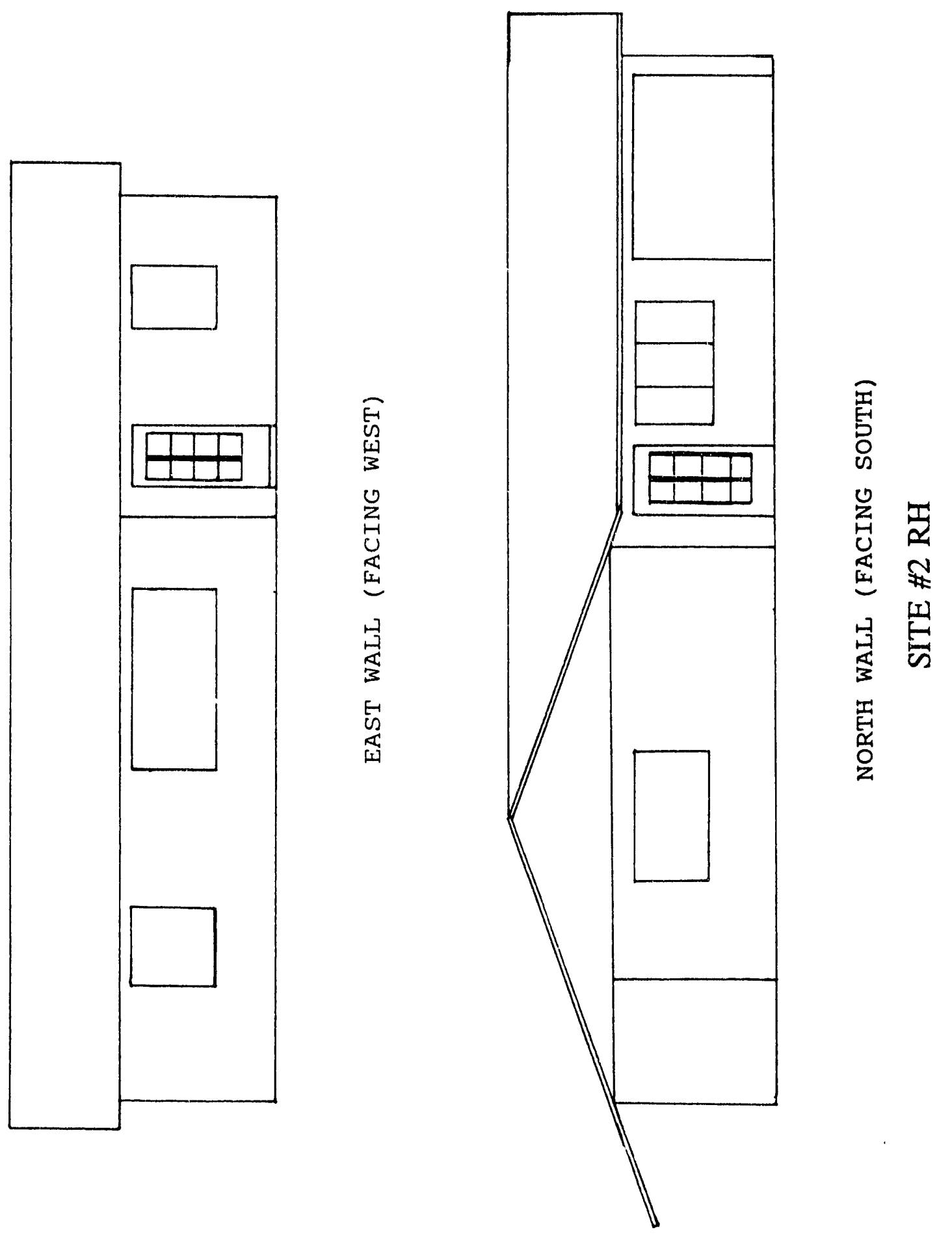

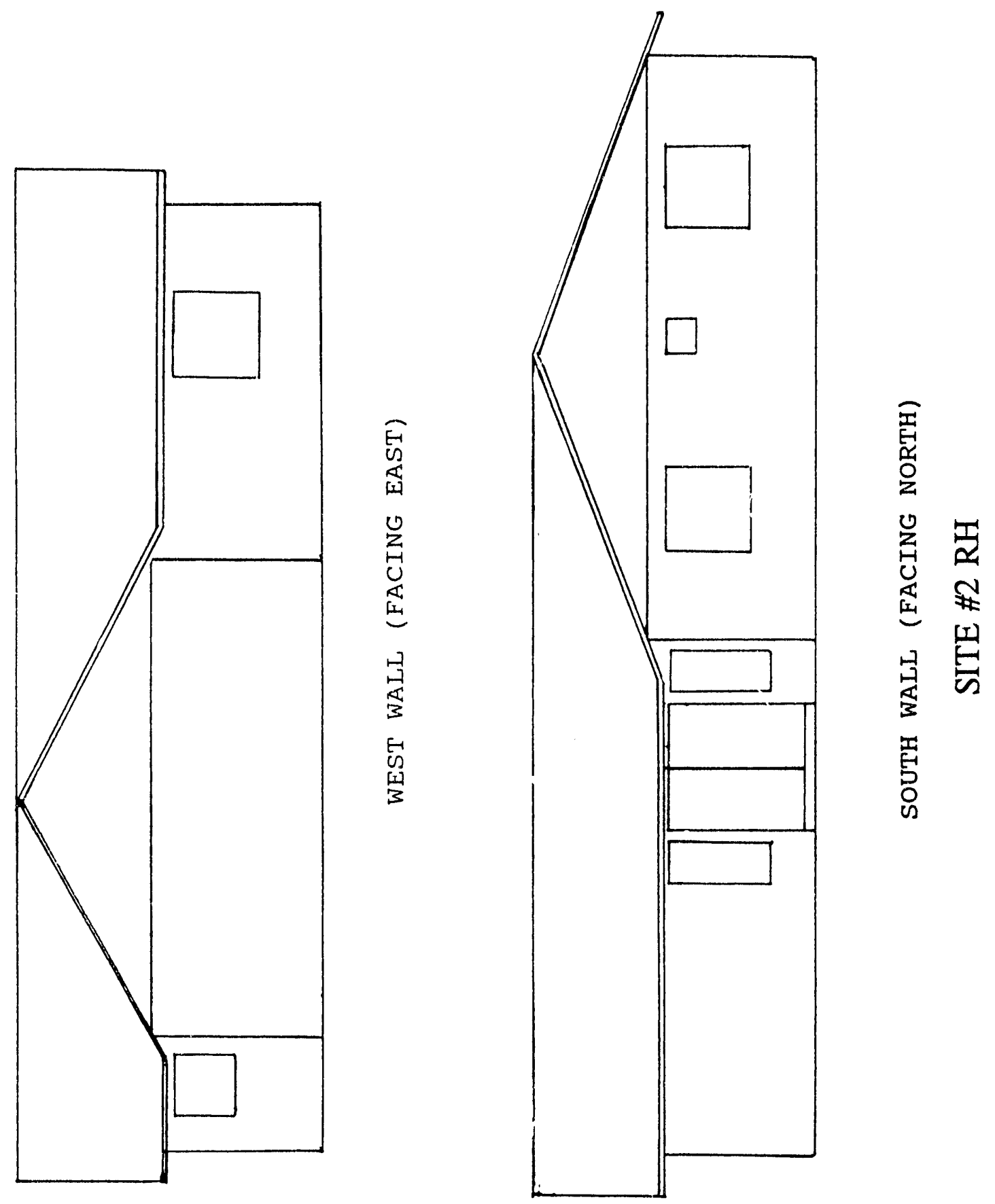
INFILTRATION RATE EVALUATION FACTORS

\begin{tabular}{|c|c|c|c|}
\hline $\begin{array}{l}\text { Building } \\
\text { component }\end{array}$ & Good & Average & Poor \\
\hline $\begin{array}{l}\text { Winoons } \\
\text { and } \\
\text { doors }\end{array}$ & $\begin{array}{l}\text { Window and door frames } \\
\text { caulked. Window and door } \\
\text { sashes well fitting and weather. } \\
\text { stripped or storm windows and } \\
\text { doors with good fit. }\end{array}$ & $\begin{array}{l}\text { Window and door frames } \\
\text { caulked or window and door } \\
\text { sashes weatherstripped or } \\
\text { poorly fitting storm doors and } \\
\text { windows. }\end{array}$ & $\begin{array}{l}\text { No caulking on window and } \\
\text { door frames. No weather- } \\
\text { stripping. No storm doors or } \\
\text { windows. }\end{array}$ \\
\hline $\begin{array}{l}\text { Walls and } \\
\text { electrical } \\
\text { outlets }\end{array}$ & 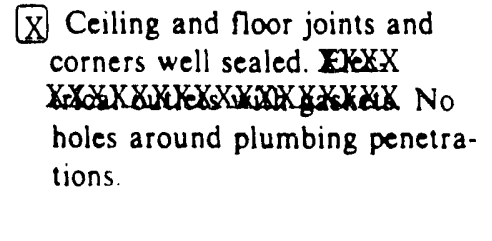 & $\begin{array}{l}\text { Some cracks in ceiling and } \\
\text { foor joints and corners. No } \\
\text { gaskets on electrical outlets. } \\
\text { Fewer than three plumbing } \\
\text { penetrations with holes } \\
\text { around them. }\end{array}$ & $\begin{array}{l}\text { Many cracks in ceiling, } \\
\text { floor joints, and corners. No } \\
\text { gaskets on electrical outlets. } \\
\text { Three or more plumbing } \\
\text { penetrations with holes } \\
\text { around them. }\end{array}$ \\
\hline $\begin{array}{l}\text { Atlic } \\
\text { noor } \\
\text { (ceiling) }\end{array}$ & $\begin{array}{l}\text { Xo cracks in attic foor. No } \\
\text { air shafts around flues. No } \\
\text { holes around ducts, pipes, or } \\
\text { wiring penetrating attic floor. } \\
\text { No recessed light fixtures. No } \\
\text { trap door or weatherstripped } \\
\text { trap door to attic. }\end{array}$ & $\begin{array}{l}\text { Some cracks in attic floor. } \\
\text { No air shafts around flues. } \\
\text { Some holes around ducts, pipes, } \\
\text { or wiring penetrating attic floor. } \\
\text { Fewer than three recessed light } \\
\text { fixtures. Unweatherstripped } \\
\text { trap door to attic. }\end{array}$ & $\begin{array}{l}\text { Many cracks in attic floor. } \\
\text { Air shafts around flues. } \\
\text { Holes around ducts, pipes, or } \\
\text { wiring penetrating attic floor. } \\
\text { More than three recessed light } \\
\text { fixtures. Uncovered attic } \\
\text { access. }\end{array}$ \\
\hline $\begin{array}{l}\text { Heating } \\
\text { system } \\
\text { and water } \\
\text { heater }\end{array}$ & $\begin{array}{l}\text { Both furnace and water } \\
\text { heater electric. If fossil fuel } \\
\text { fired, both in unconditioned } \\
\text { space. }\end{array}$ & $\begin{array}{l}\text { One fossil fuel-fired unit in } \\
\text { living space with vent } \\
\text { damper. The other in uncondi- } \\
\text { tioned space. }\end{array}$ & $\begin{array}{l}\text { At least one fossil fuel-fired } \\
\text { unit in living space without } \\
\text { vent damper. }\end{array}$ \\
\hline $\begin{array}{l}\text { Fireplace } \\
\text { or wood } \\
\text { stove }\end{array}$ & $\begin{array}{l}\text { Xealed combustion wood } \\
\text { stove or fireplace with well. } \\
\text { fitting damper and glass doors } \\
\text { or no fircplace }\end{array}$ & $\begin{array}{l}\text { Poorly sealed wood stove } \\
\text { or fireplace with either well- } \\
\text { fitting damper or glass doors. }\end{array}$ & $\begin{array}{l}\text { Both a wood stove and fire- } \\
\text { place or a fireplace with } \\
\text { poorly fitting damper and no } \\
\text { glass doors. }\end{array}$ \\
\hline $\begin{array}{l}\text { Ductwork } \\
\text { and } \\
\text { noor }\end{array}$ & $\begin{array}{l}\text { No ductwork and few noor } \\
\text { penetrations or all ductwork } \\
\text { in conditioned space and } \\
\text { no noor penetration. }\end{array}$ & $\begin{array}{l}\square \text { Ductwork in conditioned } \\
\text { basement and few noor } \\
\text { penetrations. }\end{array}$ & 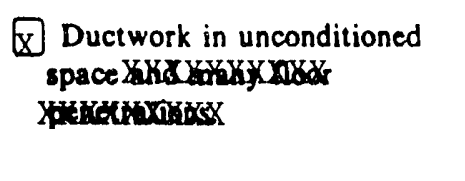 \\
\hline $\begin{array}{l}\text { Vents in } \\
\text { conditioned } \\
\text { space }\end{array}$ & $\begin{array}{l}\square \text { No undampered vents and } \\
\text { fewer than three dampered } \\
\text { vents. }\end{array}$ & $\begin{array}{l}\text { Fewer than three undampered } \\
\text { vents or at least three } \\
\text { dampered vents. }\end{array}$ & $\begin{array}{l}\square \text { More than three undampered } \\
\text { vents. }\end{array}$ \\
\hline Lifestyle & $\begin{array}{l}\square \text { Fewer than six entrances and } \\
\text { exits per day. }\end{array}$ & $\begin{array}{l}\text { Six to thirteen entrances } \\
\text { and exits per day. }\end{array}$ & $\begin{array}{l}\text { More than thirteen } \\
\text { entrances and exits per day. }\end{array}$ \\
\hline
\end{tabular}




\section{POST-RETROFIT AUDIT DATA FORM}

Retrofit program name Monitoring Conservative Retrofits in S.F.B

Sponsoring organization(s) DOE/FPL/State of FL

Experimental site designation

$\mathrm{RH}$ 非 2

Interviewer name

CSR

Date of interview $11 / 22 / 92$

1. General information from householder

1. Name Rose Hirt

2. Street address

City State

3. Phone number

4. Ow'n Rent

5. If householder different from that of "Pre-Retrofit Audit," or if tenure has changed, record below:

Change in householder Date of change

Change in tenure Date of change

6. Number of occupants, by age group:

\begin{tabular}{ll}
\multicolumn{1}{c}{ Age group } & Number \\
\hline Preschool & \\
School age & \\
Adult, but not retired & 3 \\
Retired &
\end{tabular}

How many are generally home all day?

Weekday $\frac{1}{3-4}$
Weekend

II. Significant changes since pre-retrofit audit

(The remaining questions refer to the time since the energy-saving improvements were made to the house).

1. Have there been any important changes or additions to the house, such as (check appropriate): No

Rooms added Siding added

Garage/carport added Exterior paint 
Porch added New roof

Other (specify)

2. Other than changes of this program, has the main heating or cooling system been changed, such as (check appropriate): No

New furnace/boiler

New air conditioner

New' heat pump

Modified furnace/boiler (specify)

Other (specify)

3. Are the same number of rooms still heated and cooled? (If not, specify) Yes

4. Have any space heaters been added/subtracted? (Fireplaces, wood stoves, electric, fossil fuel, etc.) Yes No $\mathrm{X}$

If so, specify

Has the use of these heaters changed during this last winter? Not app. Used more this winter _ Less _ Same _ Do not know

5. Has the hot water heating system changed? Yes _ No $\mathrm{X}$ If so, specify

6. Have any major appliances been added/subtracted? (Stoves, ovens, washers, dryers, etc.) No

If so, specify

7. Is there: An attic fan No Whole house fan No If so, has the use $c$, 'hese fans changed during this last summer? Used more this summer _ Less _ Same _ Do not know

8. Other than changes of this program, have any other energy-saving changes been installed this past year? Yes $\underline{X}$ No If yes, specify:

Ceiling insulation added

Wall insulation added Weatherstripping

Storm windows added Caulking

Storm doors added Hot water flow restrictors

Other (specify) $\frac{\text { Windows replaced in North B/R. Bay window installed }}{\text { in LR (mid-October) }}$ 
9. Have you or any family members taken up any new hobbies during the past year that require the use of large electrical or gas equipment?

Yes No $\mathrm{X}$

If so, specify 


\section{PRE-RETROFIT AUDIT DATA FORM}

Retrofit program name Monitoring Conservative Retrofits in Single Family Bldgs Sponsoring organization(s) DOE/FPL/State of FL

Experimental site designation WS \# 3

Interviewer name $\mathrm{CSR}$

Date of interview

$6 / 21 / 91$

I. General information from householder

1. Name W.H. Schick

2. Street address 1688 Shore Drive City Merritt Island State FL 32952

3. Phone number (407) 452-2387

4. Number of occupants, by age group:

\begin{tabular}{ll}
\multicolumn{1}{c}{ Age group } & Number \\
\hline Preschool & \\
School age & \\
Adult, but not retired & \\
Retired & -2
\end{tabular}

How many are generally home all day?

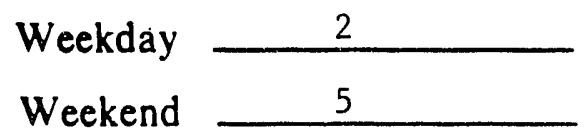

5. Approximate age of house 28 years

6. Names of utility distributors:

Electric FPL

Gas

Oil

(Note: Auditor should have homeowner sign a release authorizing release by these utilities of fuel bills for preceding two years.)

7. Ow'n Rent

8. Length of time at this address $\frac{28}{28}$ years

II. General description of house

1. Type: S-F detached $\mathrm{X}$ S-F attached

Mobile home Other (specify) 
2. Style: One-story $\mathrm{X}$ Two-story One-and-a-half-story

Split-level Crawl space

Slab $\mathrm{X}$

3. Foundation: Basement Other (specify)

4. Exterior colors: Roof tile white Walls lime

5. Layout, dimensions, orientation: Use attached House Sketch Sheet.

6. Percentagè of each floor that is conditioned (heated or cooled):

\begin{tabular}{lccc} 
Floor of house & $\begin{array}{c}\text { Percentage } \\
\text { heated }\end{array}$ & $\begin{array}{c}\text { Percentage } \\
\text { cooled }\end{array}$ & NA \\
\hline Basement & & & $\mathrm{X}$ \\
First floor & $100 \%$ & $100 \%$ & $\mathrm{X}$ \\
Second floor & & & $\mathrm{X}$ \\
Third floor & & & $\mathrm{X}$ \\
Other (specify) & & &
\end{tabular}

7. Infiltration: Use attached Infiltration Rate Evaluation Factors sheet.

8. Rooms typically closed off None

III. Major appliances

1. Domestic hot water

Type: Elec. resistance $\mathrm{X} \quad$ Heat pump hot water

Gas Solar

Other (specify)

Nameplate information, as available:

Manufacturer Kenmore

Model 153.316540

Tank capacity' (gallons) $52 \mathrm{gal}$ 3800 watt

SEER

Tank insulation ( $R$-value)

Location Garage

Hot water temperature (measured with thermometer) see data ${ }^{\circ} \mathrm{F}$

2. Solar devices

None $X \quad$ Domestic hot water Swimming pool heater

Space heating Other (specify) 
3. Other major appliances, by fuel type:

\begin{tabular}{|c|c|c|c|c|}
\hline Appliance & None & Electric & Gas & $\begin{array}{l}\text { Other } \\
\text { (specify) }\end{array}$ \\
\hline Cooking range/oven & & $\mathrm{x}$ & & \\
\hline Clothes washer & & $\mathrm{x}$ & & \\
\hline Clothes dryer & & $\mathrm{x}$ & & \\
\hline Refrigerator/freezer & & 2 & & \\
\hline Separate freezer & & & & \\
\hline Conventional oven & & $\mathrm{x}$ & & \\
\hline Microwave oven & & $\mathrm{x}$ & & \\
\hline Dishwasher & & $\mathrm{x}$ & & \\
\hline Whole house fan & & & & \\
\hline Altic fan & & & & \\
\hline Water bed heater & & & & \\
\hline Other & & & & \\
\hline
\end{tabular}

IV. Ceiling area and insulation

(If different parts of the ceiling have different insulation, treat each of these sub-areas separately.)

\begin{tabular}{cccc} 
Suh-area & $\begin{array}{c}\text { Area } \\
\left(\mathrm{ft}^{2}\right)\end{array}$ & $\begin{array}{c}\text { Insulation } \\
(\mathrm{R})\end{array}$ & $\begin{array}{c}\text { Insulation } \\
\text { type }\end{array}$ \\
\hline 1. & 1768 & $<7$ & batts \\
2. & & & \\
3. & & & \\
Total 1768 & & & \\
\end{tabular}

V. Wall area and insulation

(If different parts of wall have different materials, treat each of these sub-areas separately.)

Exterior wall construction: (1)Wood: (2) Brick; (3) Stone; (4) Stucco;

(5) Concrete block (6) Metal;

(7) Other (specify)

\begin{tabular}{ccccc} 
Sub-area & $\begin{array}{c}\text { Area } \\
\left(\mathrm{ft}^{2}\right)\end{array}$ & $\begin{array}{c}\text { Insulation } \\
(\mathrm{R})\end{array}$ & $\begin{array}{c}\text { Exterior wall } \\
\text { construction }\end{array}$ & $\begin{array}{c}\text { Insulation } \\
\text { type }\end{array}$ \\
\hline
\end{tabular}

1. $1853 \mathrm{ft}^{2}$ unk block

2.

3.

Total $1853\left(\mathrm{ft}^{2}\right)$ 
VI. Floor area and insulation (bottom floor only)

(If different parts of floor have different materials, treat each of these sub-areas separately.)

Sub-area $\begin{array}{ccccc}\text { Area } & \text { Insulation } \\ \left(\mathrm{ft}^{2}\right) & (\mathrm{R}) & \begin{array}{c}\text { Percent } \\ \text { carpeted }\end{array} & \begin{array}{c}\text { What is below? } \\ \text { (slab, crawl space, } \\ \text { basement?) }\end{array} & \begin{array}{c}\text { Insulation } \\ \text { type }\end{array}\end{array}$

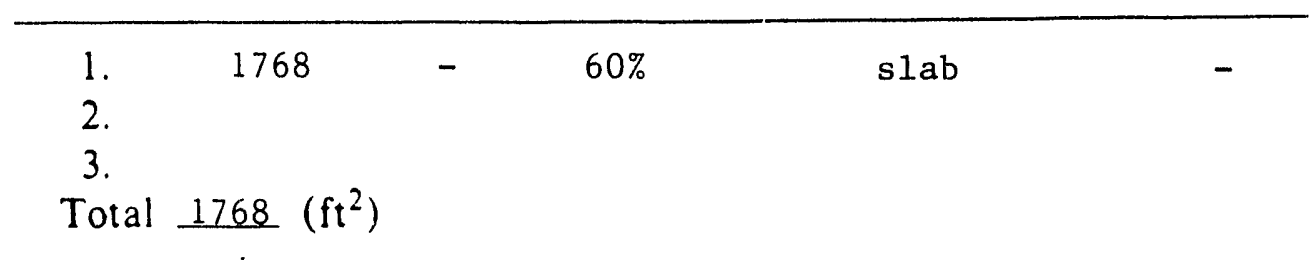

VII. W'indows and glass doors

\begin{tabular}{|c|c|c|}
\hline Orientation & $\begin{array}{c}\text { Single-pane } \\
\text { area }\end{array}$ & $\begin{array}{c}\text { Double-pane } \\
\text { area }\end{array}$ \\
\hline
\end{tabular}

Front of $\quad 65.5 \mathrm{ft}^{2} \quad 65.5 \mathrm{ft}^{2}$

house

Left side $\quad 45.0 \mathrm{ft}^{2} \quad 45.0 \mathrm{ft}^{2}$

of house

$45.0 \mathrm{ft}^{2} \quad 45.0 \mathrm{ft}^{2}$

Right side $\quad 58.5 \mathrm{ft}^{2} \quad 58.5 \mathrm{ft}^{2}$

of house

Back of $\quad 75.0 \mathrm{ft}^{2} \quad 75.0 \mathrm{ft}^{2}$

house

VIII. Doors (non-glass)

\begin{tabular}{lccc} 
Orientation & $\begin{array}{c}\text { Regular } \\
\text { door } \\
\text { area }\end{array}$ & $\begin{array}{c}\text { Regular door with } \\
\text { storm door or } \\
\text { thermally insulated } \\
\text { door area }\end{array}$ & $\begin{array}{c}\text { Total } \\
\text { area }\end{array}$ \\
\hline $\begin{array}{l}\text { Front of house } \\
\text { Left side of house }\end{array}$ & 36 & - & 36 \\
Right side of house & -- & -- \\
Back of house & 36 & -- \\
$\quad$ Totals & 72 & 36 \\
\hline
\end{tabular}


IX. Heating and cooling systems

1. Main heating system type:

Gas furnace

Gas boiler (steam)

Gas boiler (hot water)

Oil furnace

Oil boiler (steam) Oil boiler (hot water)

Elec. furnace Elec. ceiling Elec. baseboard

Central heat pump $\mathrm{X}$

Window heat pump

Other (specify)

If oil fired, has a flame retention head burner been installed?

Yes No

If oil toiler, is it wet base or dry base

Approximate age of system years

Location of unit within house Air Handler in garage

Is this a converted coal unit? Yes No Heating value of fuel: Gas

Oil

Nameplate information, as available:

Manufacturer see $A / C$

Model

Input capacity Btu/hr

Output capacity Btu/hr

SEER

Is the pilot light turned off in the spring? Yes No N/A $x$ Energy efficiency devices present:

Vent damper IID Other (specify)

2. Distribution system

No ductwork or piping Hydronic (pumped) Hydronic (gravity)

Baseboard Forced-air $\mathrm{X}$

Other (specify) 
If ductwork or piping present:

Total length of ducts or piping $130 \mathrm{ft}$

Percentage of ducts or piping in unconditioned (non-living) spaces $100 \%$ Are ducts or piping in unconditioned spaces insulated?

Yes $\mathrm{X}$ No _ If yes, approximate R-value 5

3. Main cooling system type:

None Window $\mathrm{A} / \mathrm{C}$ Central A/C

Evaporative cooler Central heat pump $\mathrm{x}$

Window heat pump Other (specify)

Approximate age of system 10 years

Nameplate information, as available

Manufacturer Carrier $380 \mathrm{~F} 036300$

Model (A/H) 40 QB 042300 (Compressor Unit) 38 QF 036300

Input capacity $\mathrm{Btu} / \mathrm{hr}$

Output capacity 36000 $\mathrm{But} / \mathrm{hr}$

SEER

Location(s) within house AH in garage

4. Secondary heating systems: None

No. fireplaces

No. fireplace inserts

No. wood stoves

No. electric space heaters

No. fossil fuel space heaters

Other (specify)

How often are these used?

5. Thermostat:

None Normal $\mathrm{X}$

Clock Other (specify)

Location within house 
$X$. Significant external loads:

Irrigation pumps

Welder

Outside lights Other (specify)

Frequency of use 3:00 AM to 6:00 PM daily

XI. Experimental retrofit installation:

Description Insulation increased to $\mathrm{R} 30$

Actual installation cost $\$-550.00$ 


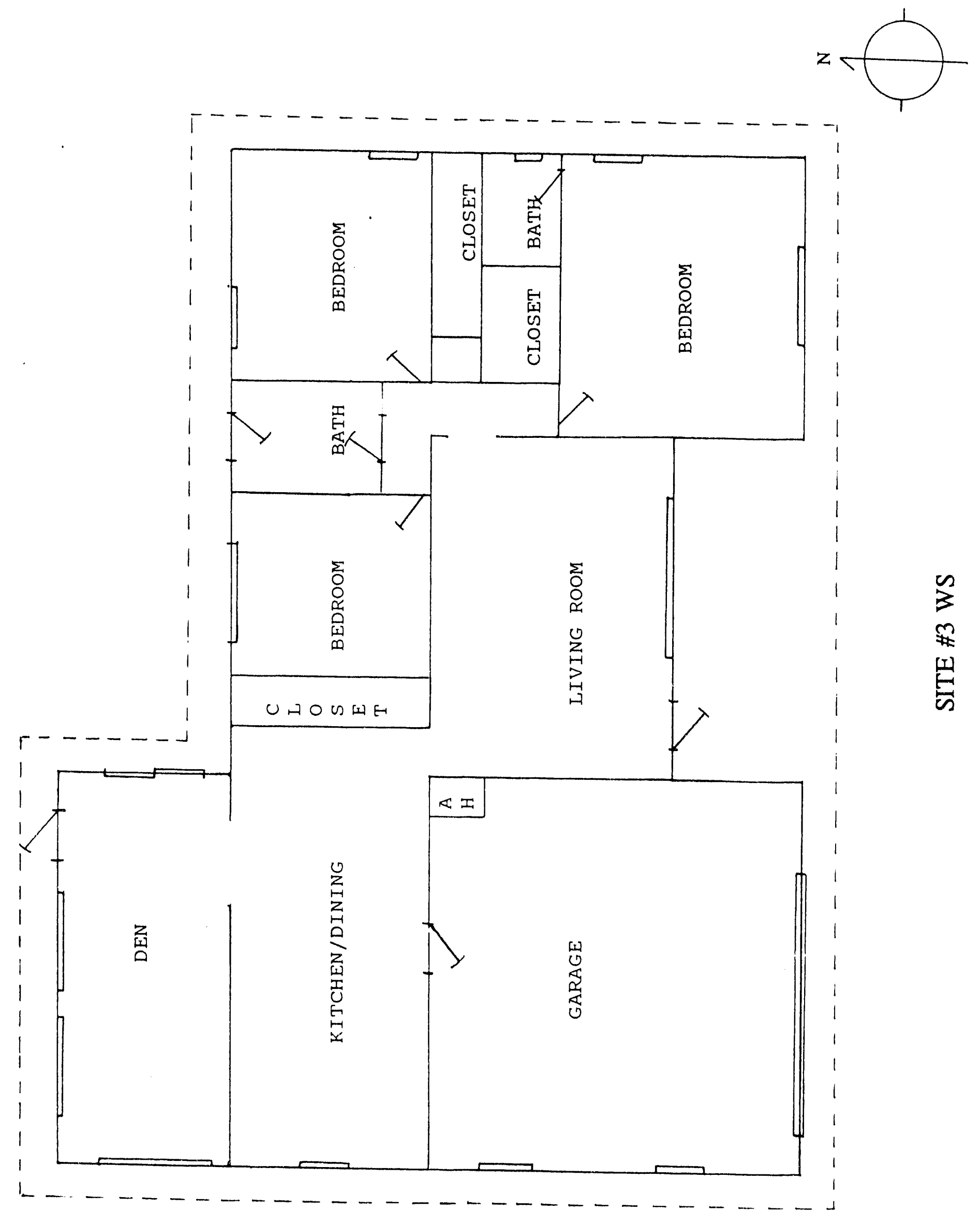



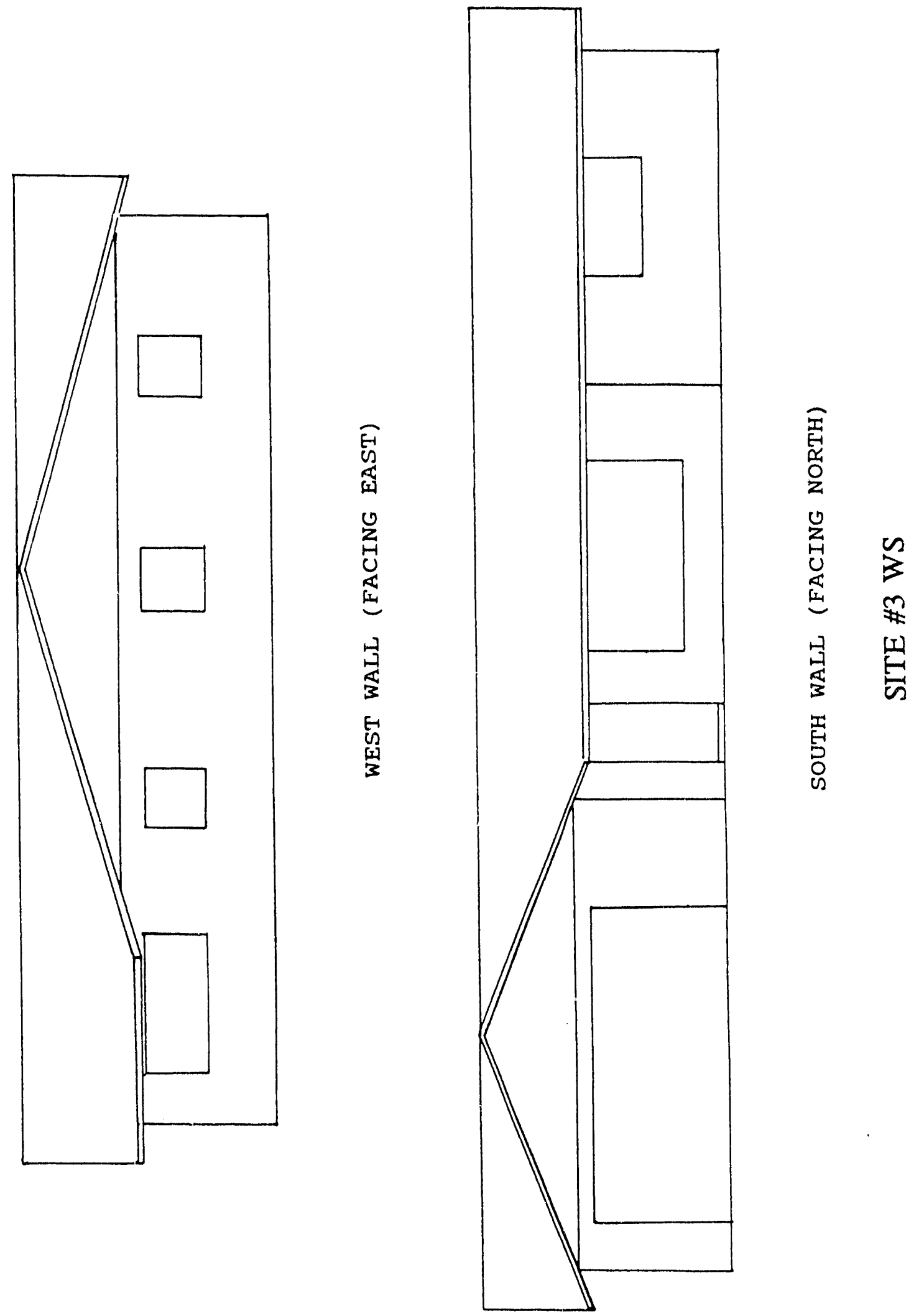

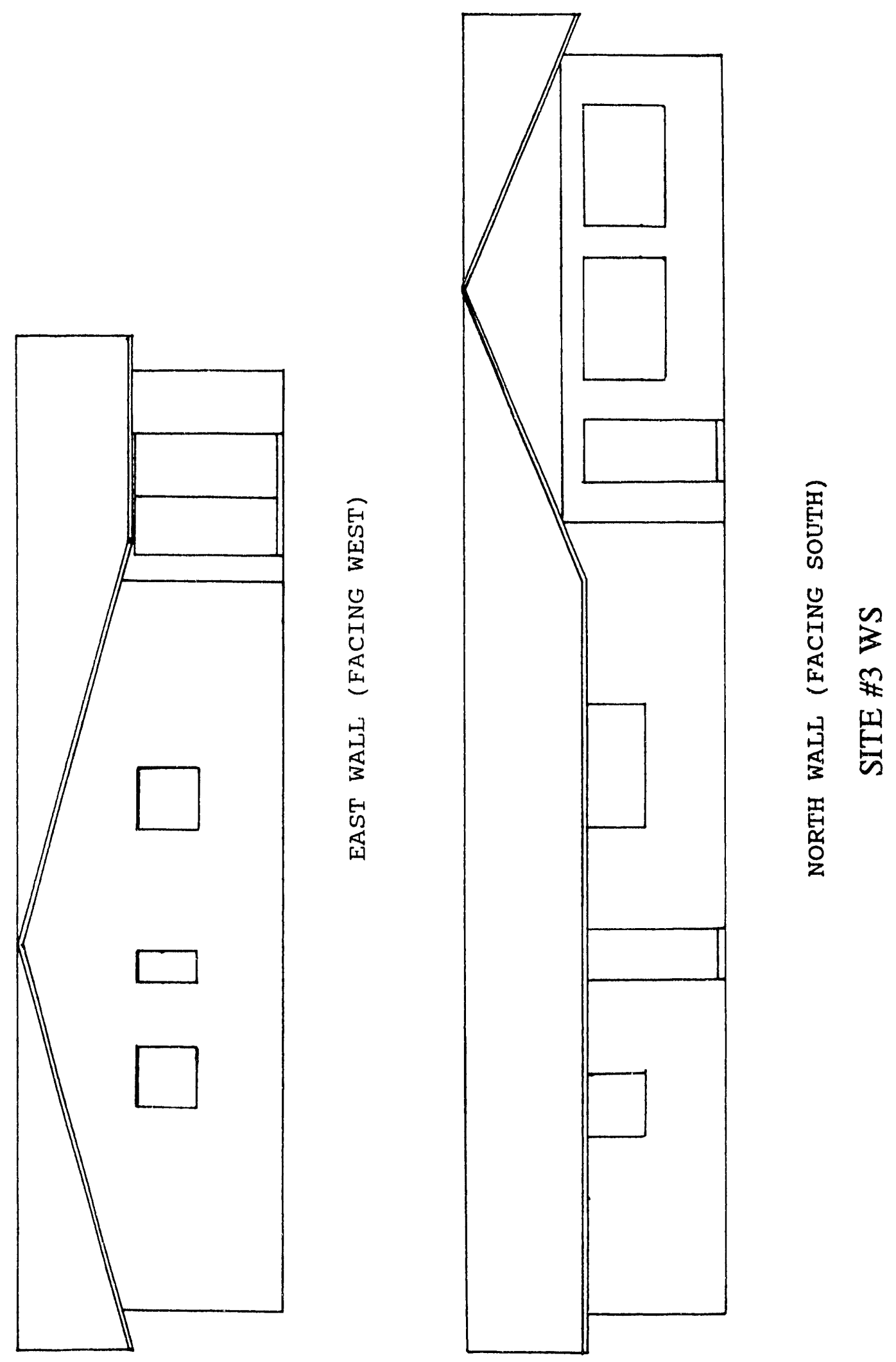
INFILTRATION RATE EVALUATION FACTORS

\begin{tabular}{|c|c|c|c|}
\hline $\begin{array}{l}\text { Building } \\
\text { component }\end{array}$ & Good & Average & Poor \\
\hline $\begin{array}{l}\text { Winonus } \\
\text { and } \\
\text { doors }\end{array}$ & $\begin{array}{l}\text { Xindow and door frames } \\
\text { caulked. Window and door } \\
\text { sashes well fitting and weather- } \\
\text { stripped or storm windows and } \\
\text { doors with good fit. }\end{array}$ & $\begin{array}{l}\text { Window and door frames } \\
\text { caulked or window and door } \\
\text { sashes weatherstripped or } \\
\text { poorly fitting storm doors and } \\
\text { windows. }\end{array}$ & $\begin{array}{l}\text { No caulking on window and } \\
\text { door frames. No weather- } \\
\text { stripping. No storm doors or } \\
\text { windows. }\end{array}$ \\
\hline $\begin{array}{l}\text { Walls and } \\
\text { electrical } \\
\text { outlets }\end{array}$ & $\begin{array}{l}\text { Xeiling and floor joints and } \\
\text { corners well sealed. Elec- } \\
\text { trical outlets with gaskets. No } \\
\text { holes around plumbing penetra- } \\
\text { tions. }\end{array}$ & $\begin{array}{l}\text { Some cracks in ceiling and } \\
\text { noor joints and corners. No } \\
\text { gaskets on electrical outlets. } \\
\text { Fewer than three plumbing } \\
\text { penetrations with holes } \\
\text { around them. }\end{array}$ & $\begin{array}{l}\text { Many cracks in ceiling, } \\
\text { foor joints, and corners. No } \\
\text { gaskets on electrical outlets. } \\
\text { Three or more plumbing } \\
\text { penetrations with holes } \\
\text { around them. }\end{array}$ \\
\hline $\begin{array}{l}\text { Attic } \\
\text { noor } \\
\text { (ceiling) }\end{array}$ & $\begin{array}{l}\text { X. No cracks in attic floor. No } \\
\text { air shafts around flues. No } \\
\text { holes around ducts, pipes, or } \\
\text { wiring penetrating attic floor. } \\
\text { No recessed light fixtures. No } \\
\text { trap door or weatherstripped } \\
\text { trap door to altic. }\end{array}$ & $\begin{array}{l}\text { Some cracks in attic floor. } \\
\text { No air shafts around flues. } \\
\text { Some holes around ducts, pipes, } \\
\text { or wiring penetrating attic floor. } \\
\text { Fewer than three recessed light } \\
\text { fixtures. Unweatherstripped } \\
\text { trap door to attic. }\end{array}$ & $\begin{array}{l}\text { Many cracks in attic floor. } \\
\text { Air shafts around tlues. } \\
\text { Holes around ducts, pipes, or } \\
\text { wiring penetrating attic floor. } \\
\text { More than three recessed light } \\
\text { fixtures. Uncovered attic } \\
\text { access. }\end{array}$ \\
\hline $\begin{array}{l}\text { Heating } \\
\text { system } \\
\text { and Hater } \\
\text { heater }\end{array}$ & $\begin{array}{l}\text { XX Both furnace and water } \\
\text { heater electric. If fossil fuel } \\
\text { fired, both in unconditioned } \\
\text { space }\end{array}$ & $\begin{array}{l}\text { One fossil fuel-fired unit in } \\
\text { living space with vent } \\
\text { damper. The other in uncondi- } \\
\text { tioned space. }\end{array}$ & $\begin{array}{l}\text { At least one fossil fuel-fired } \\
\text { unit in living space without } \\
\text { vent damper. }\end{array}$ \\
\hline $\begin{array}{l}\text { Fireplace } \\
\text { or woed } \\
\text { stove }\end{array}$ & $\begin{array}{l}\text { Sealed combustion wood } \\
\text { stove or fireplace with well- } \\
\text { fitting damper and glass doors } \\
\text { or no fireplace. }\end{array}$ & $\begin{array}{l}0 \text { Poorly sealed wood stove } \\
\text { or fireplace with either well- } \\
\text { fitting damper or glass doors. }\end{array}$ & $\begin{array}{l}\text { Both a wood stove and fire- } \\
\text { place or a fireplace with } \\
\text { poorly fitting damper and no } \\
\text { glass doors. }\end{array}$ \\
\hline $\begin{array}{l}\text { Ductuork } \\
\text { and } \\
\text { noor }\end{array}$ & $\begin{array}{l}\text { No ductwork and few noor } \\
\text { penetrations or all ductwork } \\
\text { in conditioned space and } \\
\text { no foor penetration. }\end{array}$ & $\begin{array}{l}\square \text { Ductwork in conditioned } \\
\text { basement and few floor } \\
\text { penetrations. }\end{array}$ & 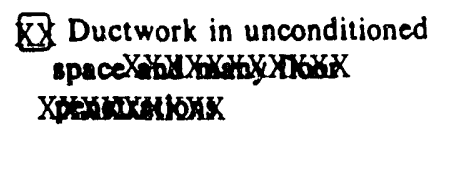 \\
\hline $\begin{array}{l}\text { Vents in } \\
\text { conditioned } \\
\text { space }\end{array}$ & $\begin{array}{l}\text { Xo undampered vents and } \\
\text { fewer than three dampered } \\
\text { vents. }\end{array}$ & $\begin{array}{l}\square \text { Fewer than three undampered } \\
\text { vents or at least three } \\
\text { dampered vents. }\end{array}$ & $\begin{array}{l}\square \text { More than three undampered } \\
\text { vents. }\end{array}$ \\
\hline Lifestyle & $\begin{array}{l}\text { Fewer than six entrances and } \\
\text { exits per day. }\end{array}$ & $\begin{array}{l}\text { X) Six to thirteen entrances } \\
\text { and exits per day. }\end{array}$ & $\begin{array}{l}\text { More than thirteen } \\
\text { entrances and exits per day. }\end{array}$ \\
\hline
\end{tabular}




\section{POST-RETROFIT AUDIT DATA FORM}

Retrofit program name Monitoring Conservative Retrofits in Single Family B1dgs Sponsoring organization(s) DOE/FPL/State of FL

Experimental site designation WS (非 3 )

Interviewer name Date of interview $9 / 15 / 92$

1. General information from householder

1. Name W.H. Schick

2. Street address

City State

3. Phone number

4. Own Rent

5. If householder different from that of "Pre-Retrofit Audit," or if tenure has changed, record below:

Change in householder Date of change

Change in tenure Date of change

6. Number of occupants, by age group:

\begin{tabular}{ll}
\multicolumn{1}{c}{ Age group } & Number \\
\hline Preschool & \\
School age \\
Adult, but not retired \\
Retired
\end{tabular}

How many are generally home all day?

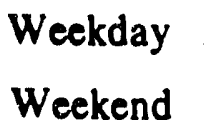

\begin{tabular}{l}
2 \\
5 \\
\hline
\end{tabular}

II. Significant changes since pre-retrofit audit NONE

(The remaining questions refer to the time since the energy-saving improvements were made to the house).

1. Have there been any important changes or additions to the house, such as (check appropriate): No

Rooms added Siding added

Garage/carport added Exterior paint 
Porch added

New roof

Other (specify)

2. Other than changes of this program, has the main heating or cooling system been changed, such as (check appropriate): No

New furnace/boiler

New air conditioner

New heat pump

Modified furnace/boiler (specify)

Other (specify)

3. Are the same number of rooms still heated and cooled? (If not, specify) Yes

4. Have any space heaters been added/subtracted? (Fireplaces, wood stoves, electric, fossil fuel, etc.) Yes

If so, specify

Has the use of these heaters changed during this last winter?

Used more this winter _ Less _ Same $\underline{x}$ _ Do not know

5. Has the hot water heating system changed? Yes _ No _ $\mathrm{X}$

If so, specify

6. Have any major appliances been added/subtracted? (Stoves, ovens, washers, dryers, etc.) No

If so, specify

7. Is there: An attic fan No Whole house fan No

If so, has the use of these fans changed during this last summer?

Used more this summer

Less

Same

Do not know

8. Other than changes of this program, have any other energy-saving cianges been installed this past year? Yes __ No _

If yes, specify:

Ceiling insulation added Weatherstripping

Wall insulation added Caulking

Storm windows added Hot water flow restrictors

Storm doors added Clock thermostat

Other (specify) 
9. Have you or any family members taken up any new hobbies during the past year that require the use of large electrical or gas equipment?

Yes - No $-\mathrm{X}$

If so, specify 


\section{PRE-RETROFIT AUDIT DATA FORM}

Retrofit program name Monitoring Conservative Retrofits in Single Family Bldgs Sponsoring organization(s) DOE/FPL/State of FL

Experimental site designation PP' (\#4)

Interviewer name CSR Date of interview $7 / 22 / 91$

I. General information from householder

1. Name Peter Postma

2. Street address

311 Cocoa Avenue

City Indialantic State

FL 32903

3. Phone number $722-2414$

4. Number of occupants, by age group:

\begin{tabular}{ll}
\multicolumn{1}{c}{ Age group } & Number \\
\hline Preschool & \\
School age & \\
Adult, but not retired \\
Retired
\end{tabular}

How many are generally home all day?

Weekday $\frac{1}{2}$
Weekend

5. Approximate age of house 29 years

6. Names of utility distributors:

Electric FPL

Gas

Oil Glover Oil

(Note: Auditor should have homeowner sign a release authorizing release by these utilities of fuel bills for preceding two years.)

7. Own $\mathrm{X}$ Rent

8. Length of time at this address 3 mos. xyodis

II. General description of house

1. Type: S-F detached $\mathrm{X}$ S-F attached Mobile home Other (specify) 
2. Style: One-story $\mathrm{X}$ Two-story Three-story

Split-level One-and-a-half-story

3. Foundation: Basement Crawl space Slab

Other (specify)

4. Exterior colors: Roof White Walls White

5. Layout, dimensions, orientation: Use attached House Sketch Sheet.

6. Percentage of each floor that is conditioned (heated or cooled):

\begin{tabular}{|c|c|c|c|}
\hline Floor of house & $\begin{array}{c}\text { Percentage } \\
\text { heated }\end{array}$ & $\begin{array}{c}\text { Percentage } \\
\text { cooled }\end{array}$ & $\mathrm{NA}$ \\
\hline $\begin{array}{l}\text { Basement } \\
\text { First floor } \\
\text { Second floor } \\
\text { Third floor } \\
\text { Other (specify) }\end{array}$ & $100 \%$ & $100 \%$ & \\
\hline \multicolumn{4}{|c|}{ Infiltration: Use attached Infiltration Rate Evaluation Factors sheet. } \\
\hline \multicolumn{4}{|c|}{ Rooms typically closed off $\quad$ None } \\
\hline
\end{tabular}

III. Major appliances

1. Domestic hot water

Type: Elec. resistance $\mathrm{X} \quad$ Heat pump hot water

Gas Solar

Other (specify)

Nameplate information, as available:

Manufacturer Craftmaster

Model CREFR52DL

Tank capacity (gallons) 52 gal

SEER

Tank insulation ( $\mathrm{R}$-value) R 12.5

Location Hal1 closet

Hot water temperature (measured with thermometer) see data ${ }^{\circ} \mathrm{F}$ 2. Solar devices

None $\underline{X}$ Domestic hot water Swimming pool heater Space heating Other (specify) 
3. Other major appliances, by fuel type:

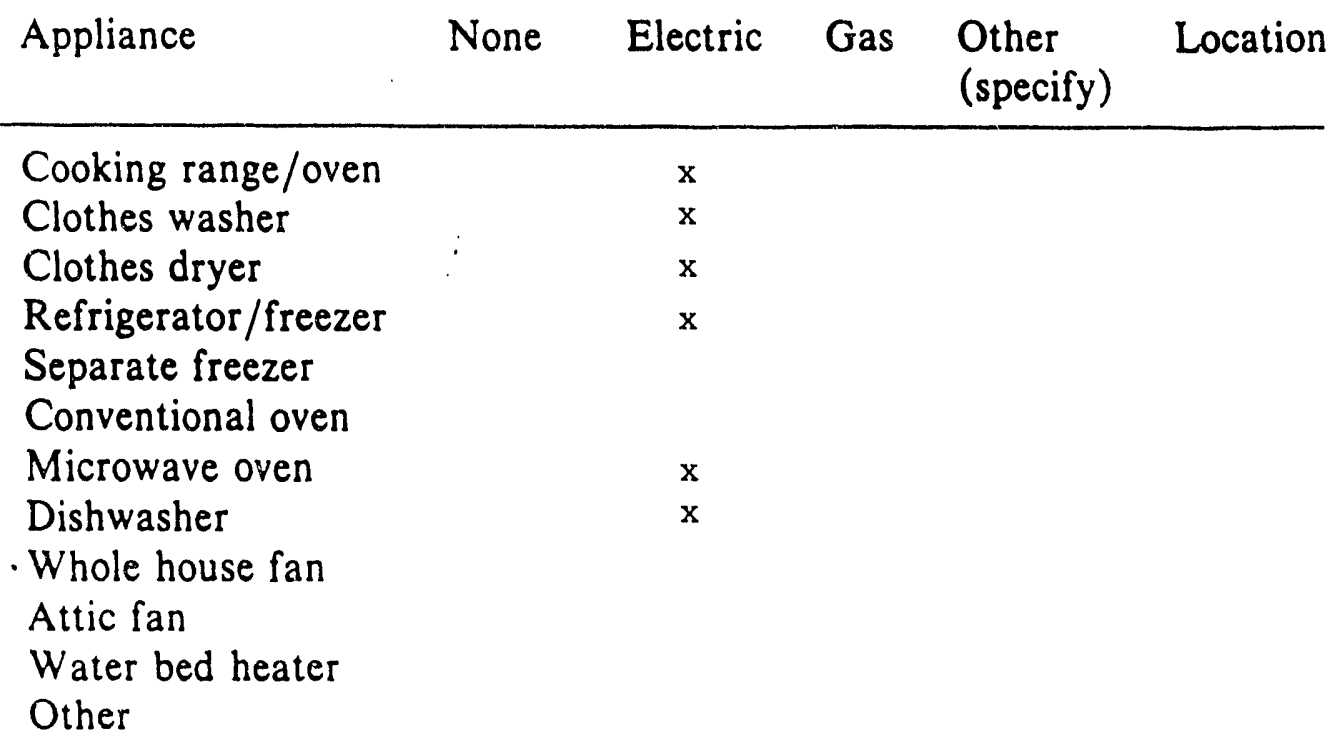

IV. Ceiling area and insulation

(If different parts of the ceiling have different insulation, treat each of these sub-areas separately.)

\begin{tabular}{lrrr} 
Sub-area & $\begin{array}{c}\text { Area } \\
\left(\mathrm{ft}^{2}\right)\end{array}$ & $\begin{array}{c}\text { Insulation } \\
(\mathrm{R})\end{array}$ & $\begin{array}{c}\text { Insulation } \\
\text { type }\end{array}$ \\
\hline 1. $1880 \mathrm{ft}^{2}$ & $<7$ & batts \\
2. & & & \\
3. & & \\
Total $\underline{1880}\left(\mathrm{ft}^{2}\right)$ & &
\end{tabular}

V. Wall area and insulation

(If different parts of wall have different materials, treat each of these sub-areas separately.)

Exterior wall construction: (1)Wood; (2) Brick; (3) Stone; (4)Stucco

(5) Concrete block (6) Metal;

(7) Other (specify)

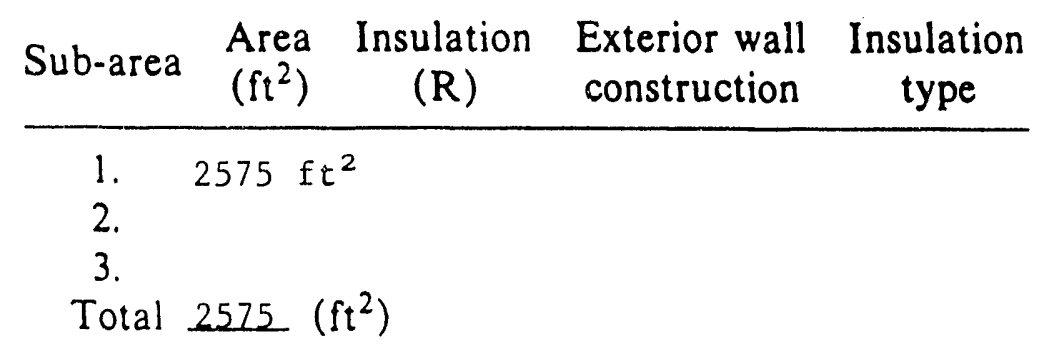


VI. Floor area and insulation (bottom floor only)

(If different parts of floor have different materials, treat each of these sub-areas separately.)

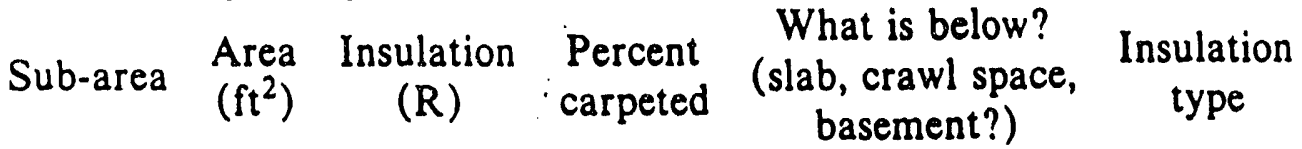

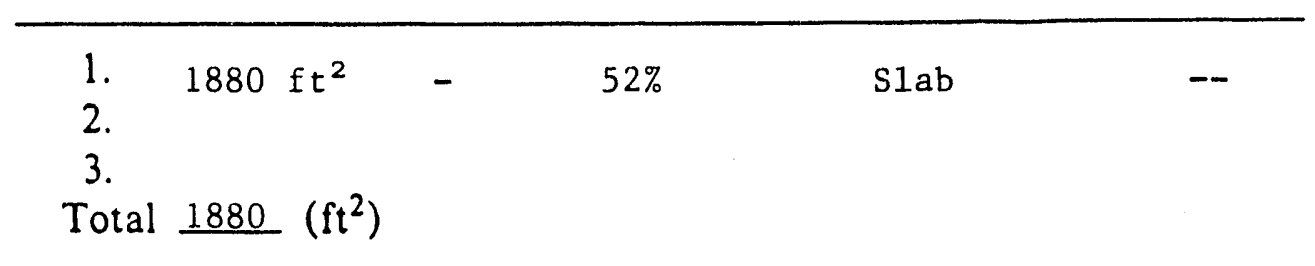

VII. Windows and glass doors

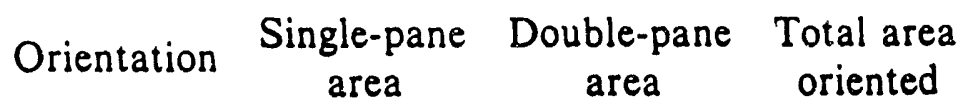

Front of $\quad 90 \mathrm{ft}^{2} \quad 90 \mathrm{ft}^{2}$

house

Left si le

$63 \mathrm{ft}^{2}$

$63 \mathrm{ft}^{2}$

of house

Right side $\quad 85 \frac{1}{2} \mathrm{ft}^{2}$

of house

$85 \frac{1}{2} \mathrm{ft}^{2}$

Back of

house

$131 \mathrm{ft}^{2}$

$131 \mathrm{ft}^{2}$

house

VIII. Doors (non-glass)

\begin{tabular}{|c|c|c|c|}
\hline Orientation & $\begin{array}{l}\text { Regular } \\
\text { door } \\
\text { area }\end{array}$ & $\begin{array}{l}\text { Regular door with } \\
\text { storm door or } \\
\text { thermally insulated } \\
\text { door area }\end{array}$ & $\begin{array}{l}\text { Total } \\
\text { area }\end{array}$ \\
\hline $\begin{array}{l}\text { Forth } \\
\text { Feft of house } \\
\text { Right side of house } \\
\text { Back of house }\end{array}$ & $f t^{2}+21$ & to garage & $63 \mathrm{ft}^{2}$ \\
\hline Totals & $63 \mathrm{ft}^{2}$ & & $63 \mathrm{ft}^{2}$ \\
\hline
\end{tabular}


IX. Heating and cooling systems

1. Main heating system type:

Gas furnace Gas boiler (steam)

Gas boiler (hot water) Oil furnace Luxaire OU100TF

Oil boiler (steam) Oil boiler (hot water)

Elec. furnace Elec. ceiling Elec. baseboard

Central heat pump Window heat pump

Other (specify)

If oil fired, has a flame retention head burner been installed?

Yes No

If oil boiler, is it wet base or dry base gun burner

Approximate age of system years

Location of unit within house Garage

Is this a converted coal unit? Yes No

Heating value of fuel: Gas

$$
\text { Oil }
$$

Nameplete information, as available:

Manuiacturer Luxaire

Model OU100TF

Input capacity 126,000

Output capacity Btu/hr

SEER

Is the pilot light turned off in the spring? Yes No N/A X Energy efficiency devices present:

Vent damper IID Other (specify)

2. Distribution system

No ductwork or piping Hydronic (gravity)

Hydronic (pumped) Forced-air $\mathrm{X}$

Baseboard Gravity system (no fans)

Other (specify) 
If ductwork or piping present:

Total length of ducts or piping $150^{\prime}$ supply; $86^{\prime}$ return

Percentage of ducts or piping in unconditioned (non-living) spaces $100 \%$

Are ducts or piping in unconditioned spaces insulated?

Yes supply No return If yes, approximate R-value $<<4$

3. Main cooling system type:

None Window $\mathrm{A} / \mathrm{C}$ Central A/C $\mathrm{X}$

Evaporative cooler Central heat pump

Window heat pump Other (specify)

Approximate age of system 8 years

Nameplate information, as available

Manufacturer York

Model H2CB048506A comp/ Rheem Evap AU 49CDUS

Input capacity $\mathrm{Btu} / \mathrm{hr}$

Output capacity $\frac{60,000}{<7}$ But/hr

SEER garage

Location(s) within house

4. Secondary heating systems:

No. fireplaces

No. fireplace inserts

No. wood stoves

No. electric space heaters

No. fossil fuel space heaters

Other (specify)

How often are these used?

\& 3 times per year

5. Thermostat:

None Normal X

Clock Other (specify)

Location within house Hallway near wall return 
$X$. Significant external loads:

Irrigation pumps

Welder

Outside lights Other (specify)

Frequency of use

XI. Experimental retrofit installation:

Description Insulation increase to $R 19$

Actual installation cost $\$$

608.00 


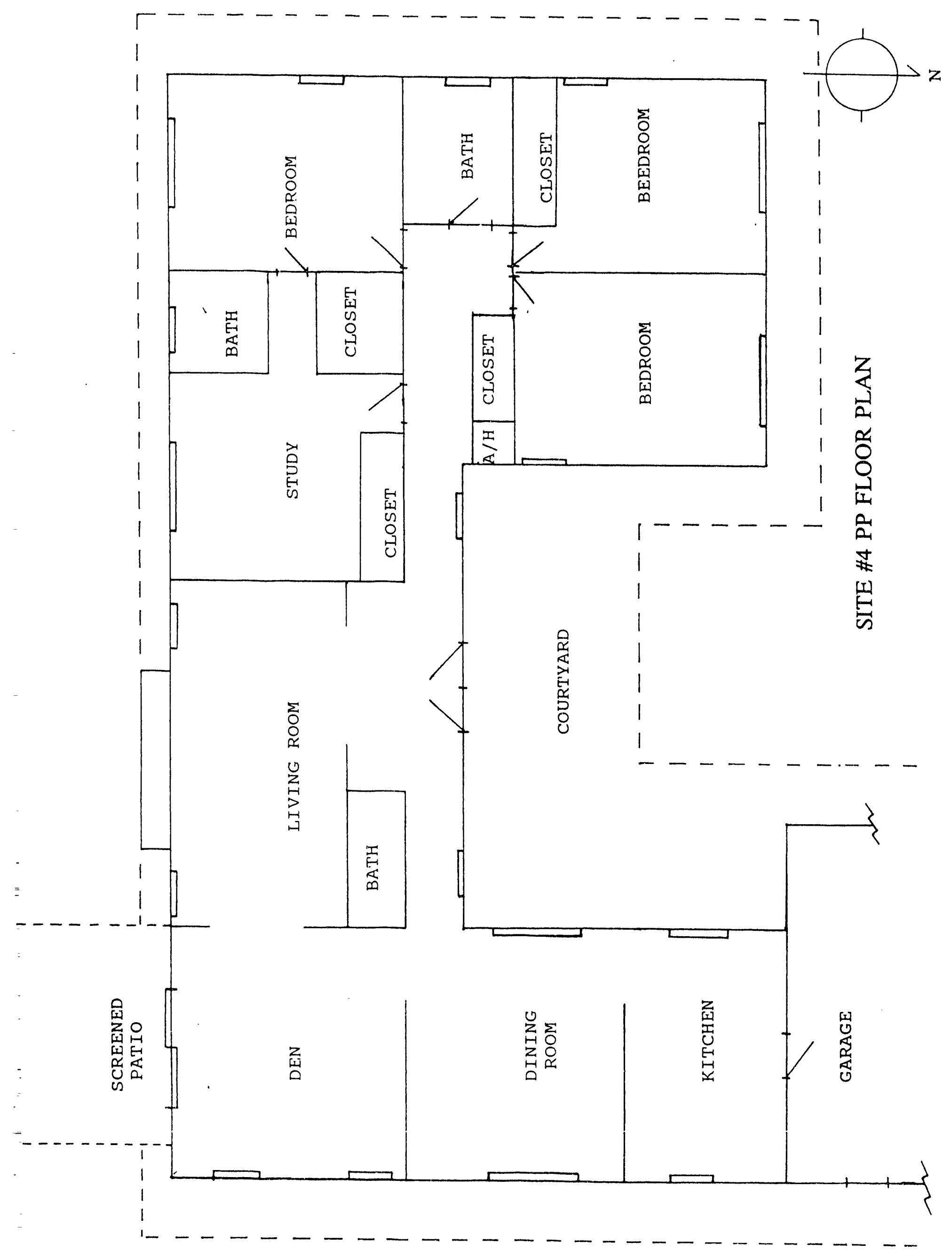



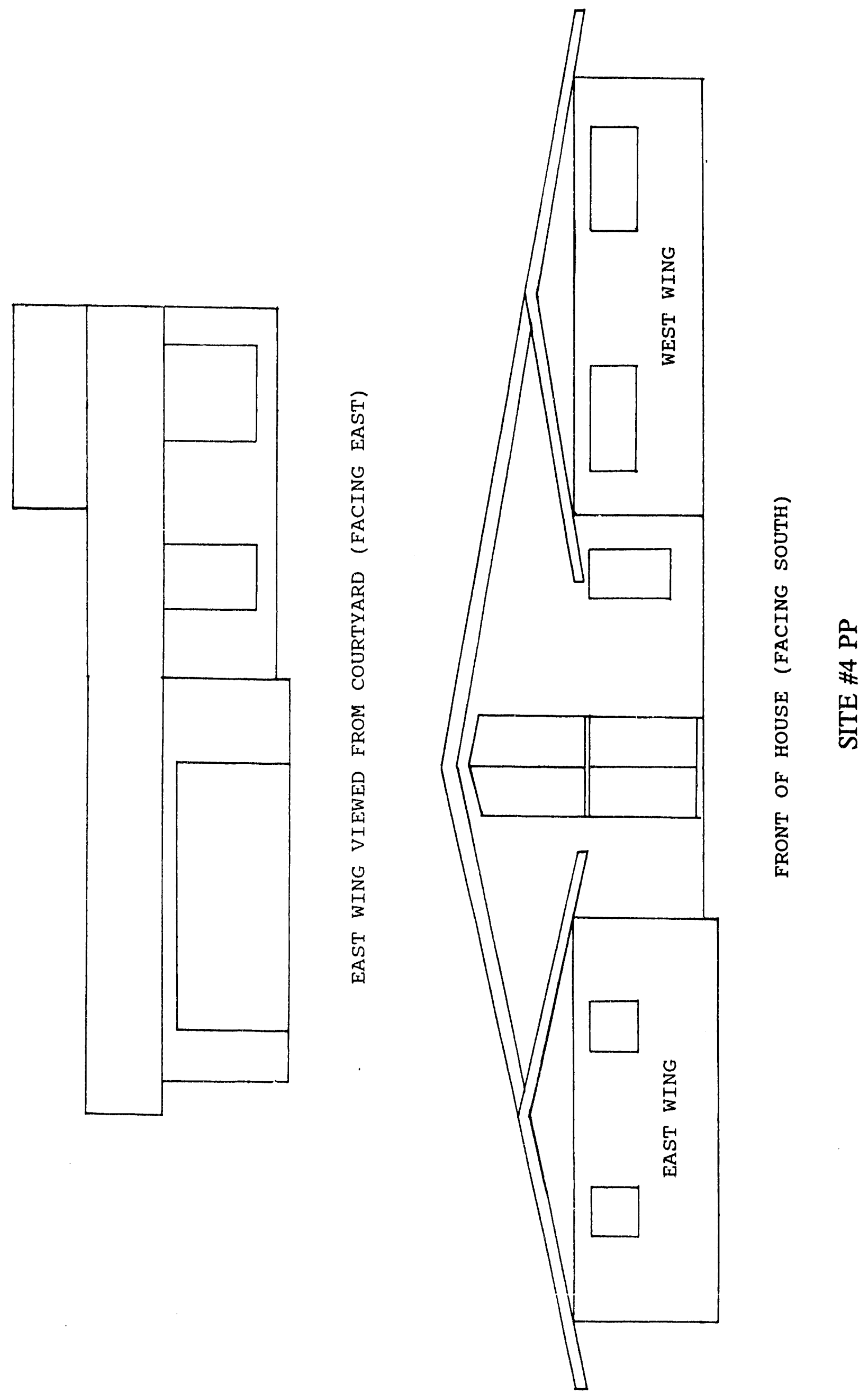


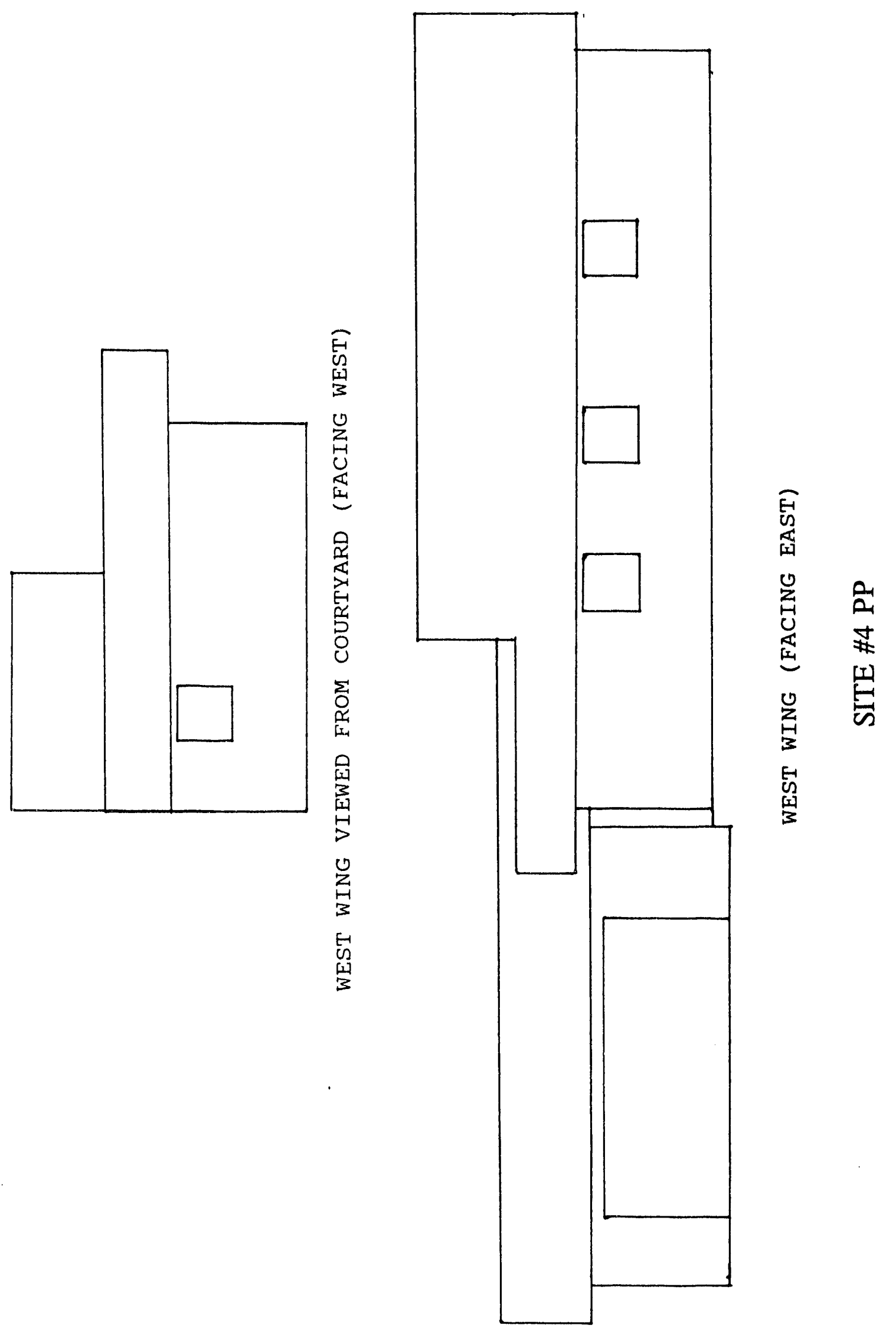



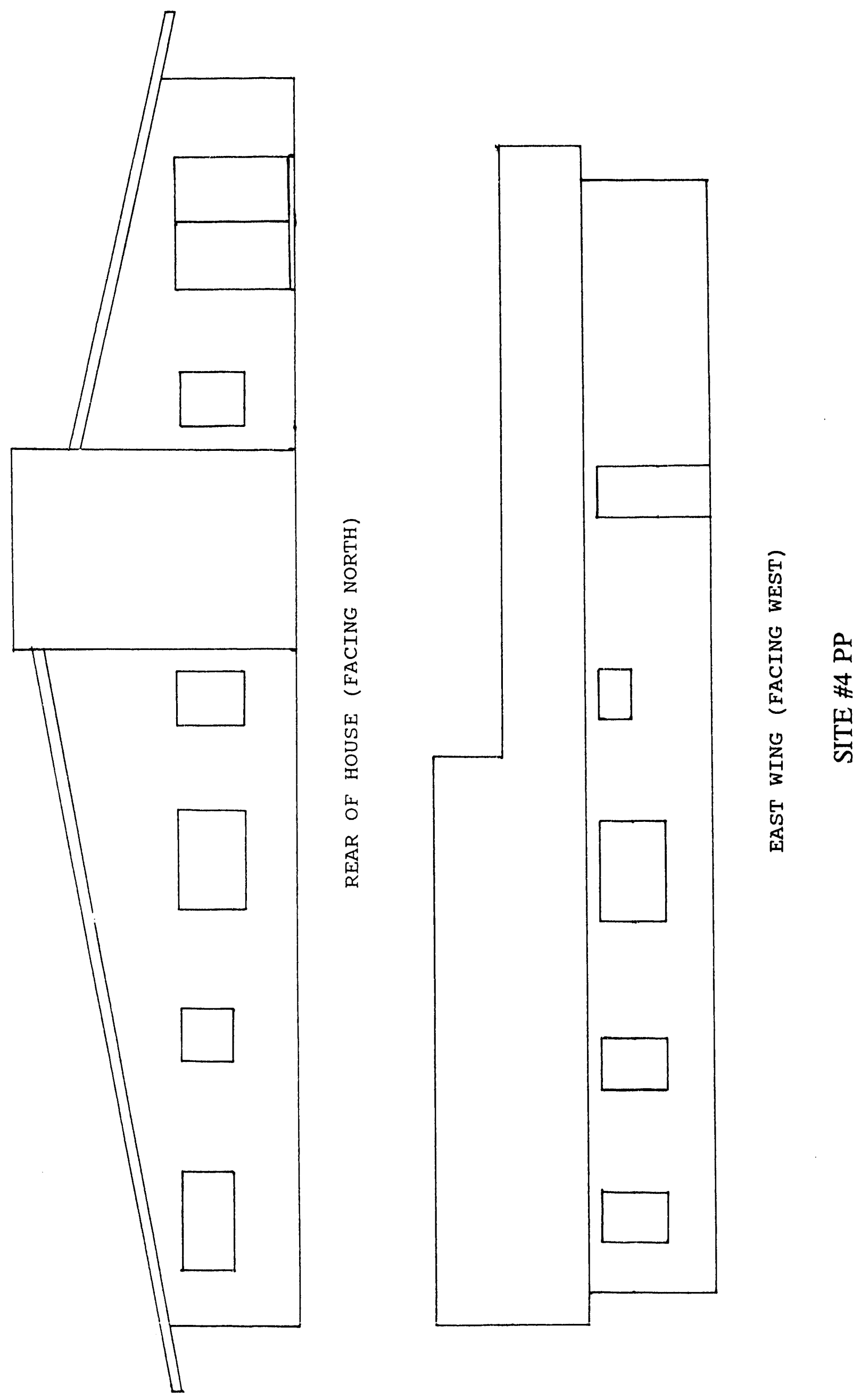
INFILTRATION RATE EVALUATION FACTORS

\begin{tabular}{|c|c|c|c|}
\hline $\begin{array}{l}\text { Building } \\
\text { component }\end{array}$ & Good & Average & Poor \\
\hline $\begin{array}{l}\text { Windows } \\
\text { and } \\
\text { doors }\end{array}$ & $\begin{array}{l}\text { X Window and door frames } \\
\text { caulked. Window and door } \\
\text { sashes well fitting and weather- } \\
\text { stripped or storm windows and } \\
\text { doors with good fit. }\end{array}$ & $\begin{array}{l}\text { Window and door frames } \\
\text { caulked or window and door } \\
\text { sashes weatherstripped or } \\
\text { poorly fitting storm doors and } \\
\text { windows. }\end{array}$ & $\begin{array}{l}\text { No caulking on window and } \\
\text { door frames. No weather- } \\
\text { stripping. No storm doors or } \\
\text { windows. }\end{array}$ \\
\hline $\begin{array}{l}\text { Walls and } \\
\text { electrical } \\
\text { outlets }\end{array}$ & $\begin{array}{l}\text { Ceiling and noor joints and } \\
\text { corners well sealed. Elec- } \\
\text { trical outlets with gaskets. No } \\
\text { holes around plumbing penetra- } \\
\text { tions. }\end{array}$ & $\begin{array}{l}\text { X) Some cracks in ceiling and } \\
\text { noor joints and corners. No } \\
\text { gaskets on electrical outlets. } \\
\text { Fewer than three plumbing } \\
\text { penetrations with holes } \\
\text { around them. }\end{array}$ & $\begin{array}{l}\text { Many cracks in ceiling, } \\
\text { Moor joints, and corners. No } \\
\text { gaskets on electrical outlets. } \\
\text { Three or more plumbing } \\
\text { penetrations with holes } \\
\text { around them. }\end{array}$ \\
\hline $\begin{array}{l}\text { Attic } \\
\text { Moor } \\
\text { (ceiling) }\end{array}$ & $\begin{array}{l}\text { No cracks in attic noor. No } \\
\text { air shafts around flues. No } \\
\text { holes around ducts, pipes, or } \\
\text { wiring penetrating attic noor. } \\
\text { No recessed light fixtures. No } \\
\text { trap door or weatherstripped } \\
\text { trap door to attic. }\end{array}$ & $\begin{array}{l}\text { Some cracks in attic floor. } \\
\text { No air shafts around flues. } \\
\text { Some holes around ducts, pipes, } \\
\text { or wiring penetrating attic floor. } \\
\text { Fewer than three recessed light } \\
\text { fixtures. Unweatherstripped } \\
\text { trap door to attic. }\end{array}$ & $\begin{array}{l}\text { Many cracks in attic floor. } \\
\text { Air shafts around flues. } \\
\text { Holes around ducts, pipes, or } \\
\text { wiring penetrating attic floor. } \\
\text { More than three recessed light } \\
\text { lixtures. Uncovered attic } \\
\text { access. }\end{array}$ \\
\hline $\begin{array}{l}\text { Heating } \\
\text { system } \\
\text { and water } \\
\text { heater }\end{array}$ & $\begin{array}{l}\text { Both furnace and water } \\
\text { heater electric. If fossil fuel } \\
\text { fired, both in unconditioned } \\
\text { space. }\end{array}$ & $\begin{array}{l}\text { One fossil fuel-fired unit in } \\
\text { living space with vent } \\
\text { damper. The other in uncondi- } \\
\text { tioned space. }\end{array}$ & $\begin{array}{l}\text { At least one fossil fuel-fired } \\
\text { unit in living space without } \\
\text { vent damper. }\end{array}$ \\
\hline 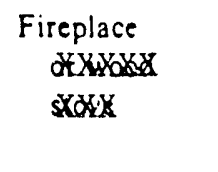 & $\begin{array}{l}8 . \text { Sealed combustion wood } \\
\text { stove or fireplace with well- } \\
\text { fitting damper and glass doors } \\
\text { or no lireplace. }\end{array}$ & $\begin{array}{l}\text { Poorly sealed wood stove } \\
\text { or fireplace with either well- } \\
\text { fitting damper or glass doors. }\end{array}$ & $\begin{array}{l}\text { Both a wood stove and fire- } \\
\text { place or a fireplace with } \\
\text { poorly fitting damper and no } \\
\text { glass doors. }\end{array}$ \\
\hline $\begin{array}{l}\text { Ductwork } \\
\text { and } \\
\text { noor }\end{array}$ & $\begin{array}{l}\text { No ductwork and few noor } \\
\text { penetrations or all ductwork } \\
\text { in conditioned space and } \\
\text { no floor penetration. }\end{array}$ & $\begin{array}{l}\text { Ductwork in conditioned } \\
\text { basement and few floor } \\
\text { penetrations. }\end{array}$ & 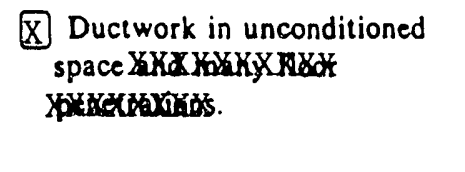 \\
\hline $\begin{array}{l}\text { Vents in } \\
\text { conditioned } \\
\text { space }\end{array}$ & $\begin{array}{l}\text { No undampered vents and } \\
\text { fever than three dampered } \\
\text { vents. }\end{array}$ & $\begin{array}{l}\text { Fewer than three undampered } \\
\text { vents or at least three } \\
\text { dampered vents. }\end{array}$ & $\begin{array}{l}\square \text { More than three undampered } \\
\text { vents. }\end{array}$ \\
\hline Lifestyle & $\begin{array}{l}\square \text { Fewer than six entrances and } \\
\text { exits per day. }\end{array}$ & $\begin{array}{l}\text { Six to thirteen entrances } \\
\text { and exits per day. }\end{array}$ & $\begin{array}{l}\text { More than thirteen } \\
\text { entrances and exits per day. }\end{array}$ \\
\hline
\end{tabular}




\section{POST-RETROFIT AUDIT DATA FORM}

Retrofit program name Monitoring Conservative Retrofits in Single Family Bldgs Sponsoring organization(s) DOE/FPL/State of FL

Experimental site designation $\quad$ PP (非)

Interviewer name CSR Date of interview $8 / 24 / 92$

I. General information from householder

1. Name Peter Postma

2. Street address City State

3. Phone number

4. Own Rent

5. If householder different from that of "Pre-Retrofit Audit," or if tenure has changed, record below:

Change in householder Date of change Change in tenure Date of change

6. Number of occupants, by age group:

\begin{tabular}{ll}
\multicolumn{1}{c}{ Age group } & Number \\
\hline Preschool & \\
School age & \\
Adult, but not retired & 2 \\
Retired &
\end{tabular}

How many are generally home all day?

Weekday $\frac{1}{2}$
Weekend

II. Significant changes since pre-retrofit audit NONE

(The remaining questions refer to the time since the energy-saving improvements were made to the house).

1. Have there been any important changes or additions to the house, such as (check appropriate): No

Rooms added Siding added

Garage/carport added Exterior paint 
Porch added New roof

Other (specify)

2. Other than changes of this program, has the main heating or cooling system been changed, such as (check appropriate): No

New furnace/boiler

New air conditioner

New heat pump

Modified furnace/boiler (specify)

Other (specify)

3. Are the same number of rooms still heated and cooled? (If not, specify) Yes

4. Have any space heaters been added/subtracted? (Fireplaces, wood stoves, electric, fossil fuel, etc.) Yes No $\mathrm{X}$

If so, specify

Has the use of these heaters changed during this last winter?

Used more this winter Less Same Do not know

5. Has the hot water heating system changed? Yes __ No _ $\mathrm{X}$ If so, specify

6. Have any major appliances been added/subtracted? (Stoves, ovens, washers, dryers, etc.) No

If so, specify

7. Is there: An attic fan No Whole house fan No

If so, has the use of these fans changed during this last summer?

Used more this summer _ Less _ Same _ Do not know _

8. Other than changes of this program, have any other energy-saving changes been installed this past year? Yes __ No X X

If yes, specify:

Ceiling insulation added Weatherstripping

Wall insulation added Caulking

Storm windows added Hot water flow restrictors

Storm doors added Clock thermostat

Other (specify) 
9. Have you or any family members taken up any new hobbies during the past year that require the use of large electrical or gas equipment?

Yes $\_$No $\quad \mathrm{X}$

If so, specify 


\section{PRE-RETROFIT AUDIT DATA FORM}

Retrofit program name Monitoring Conservative Retrofits in Single Family Bldgs Sponsoring organization(s) DOE/FPL/State of FL

Experimental site designation $R R \quad(\# 5)$

Interviewer name CSR

Date of interview

$3 / 26 / 91$

I. General information from householder

1. Name R. E. Rainsberger

2. Street address 209 Flamingo Lane

City Melbourne Beach

State

FL 32951

3. Phone number

$723-7007$

4. Number of occupants, by age group:

\begin{tabular}{lc}
\multicolumn{1}{c}{ Age group } & Number \\
\hline Preschool & \\
School age & \\
Adult, but not retired & \\
Retired &
\end{tabular}

How many are generally home all day?

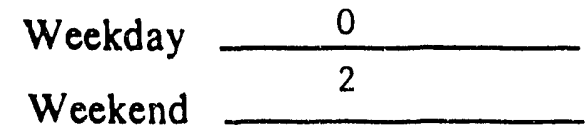

5. Approximate age of house 27 years

6. Names of utility distributors:

Electric

FPL

Gas

Oil

(Note: Auditor should have homeowner sign a release authorizing release by these utilities of fuel bills for preceding two years.)

7. Own $\mathrm{X}$ Rent

8. Length of time at this address years

II. General description of house

1. Type: S-F detached $\mathrm{X}$ Mobile home S.F attached Other (specify) 
2. Style: One-story _ $\mathrm{x}$ Two-story Three-story Split-level One-and-a-half-story

3. Foundation: Basement Crawl space Slab $\mathrm{x}$ Other (specify)

4. Exterior colors: Roof Walls

5. Layout, dimensions, orientation: Use attached House Sketch Sheet.

6. Percentage of each floor that is conditioned (heated or cooled):

\begin{tabular}{lccc} 
Floor of house & $\begin{array}{c}\text { Percentage } \\
\text { heated }\end{array}$ & $\begin{array}{c}\text { Percentage } \\
\text { cooled }\end{array}$ & NA \\
\hline $\begin{array}{l}\text { Basement } \\
\text { First floor }\end{array}$ & $92 \%$ & $92 \%$ \\
$\begin{array}{l}\text { Second floor } \\
\text { Third floor }\end{array}$ & & \\
Other (specify) & &
\end{tabular}

7. Infiltration: Use attached Infiltration Rate Evaluation Factors sheet.

8. Rooms typically closed off Florida Room

III. Major appliances

1. Domestic hot water

Type: Elec. resistance $\mathrm{x}$ Heat pump hot water

Gas Solar

Other (specify)

Nameplate information, as available:

Manufacturer Bradford White

Model $M-I-40510$

Tank capacity (gallons) 40 gal

\section{SEER}

Tank insulation ( $\mathrm{R}$-value)

Location Garage

Hot water temperature (measured with thermometer) in data set ${ }^{\circ} \mathrm{F}$

2. Solar devices

None $\mathrm{x}$ Domestic hot water Swimming pool heater 
3. Other major appliances, by fuel type:

\begin{tabular}{|c|c|c|c|c|c|}
\hline Appliance & None & Electric & Gas & $\begin{array}{l}\text { Other } \\
\text { (specify) }\end{array}$ & Location \\
\hline Cooking range/oven & & $\mathrm{x}$ & & & \\
\hline Clothes washer & & $\mathrm{x}$ & & & \\
\hline Clothes dryer & & $\mathrm{x}$ & & & \\
\hline Refrigerator/freezer & & $\mathrm{x}$ & & & \\
\hline Separate freezer & & & & & \\
\hline Conventional oven & & & & & \\
\hline Microwave oven & & $\mathrm{x}$ & & & \\
\hline Dishwasher & & $\mathrm{x}$ & & & \\
\hline Whole house fan & & & & & \\
\hline Attic fan & & & & & \\
\hline Water bed heater & & & & & \\
\hline Other & & & & & \\
\hline
\end{tabular}

IV. Ceiling area and insulation

(If different parts of the ceiling have different insulation, treat each of these sub-areas separately.)

\begin{tabular}{cccc} 
Sub-area & $\begin{array}{c}\text { Area } \\
\left(\mathrm{ft}^{2}\right)\end{array}$ & $\begin{array}{c}\text { Insulation } \\
(\mathrm{R})\end{array}$ & $\begin{array}{c}\text { Insulation } \\
\text { type }\end{array}$ \\
\hline $\begin{array}{l}\text { 1. } \\
\text { 2. }\end{array}$ & $2172 \mathrm{ft}^{2}$ & $<7$ & batts \\
3. & & & \\
Total & 2172 $\left(\mathrm{ft}^{2}\right)$ & &
\end{tabular}

V. Wall area and insulation

(If different parts of wall have different materials, treat each of these sub-areas separately.)

Exterior wall construction: (1)Wood; (2) Brick; (3) Stone; (4) Stucco;

(5) Concrete block (6) Metal;

(7) Other (specify)

\begin{tabular}{ccccc} 
Sub-area & $\begin{array}{c}\text { Area } \\
\left(\mathrm{ft}^{2}\right)\end{array}$ & $\begin{array}{c}\text { Insulation } \\
(\mathrm{R})\end{array}$ & $\begin{array}{c}\text { Exterior wall } \\
\text { construction }\end{array}$ & $\begin{array}{c}\text { Insulation } \\
\text { type }\end{array}$ \\
\hline
\end{tabular}

1. $2184.5 \mathrm{ft}^{2}$

2.

3.

Total $2184.5\left(\mathrm{ft}^{2}\right)$ 
VI. Floor area and insulation (bottom floor only)

(If different parts of floor have different 1 :aterials, treat each of these sub-areas separately.)

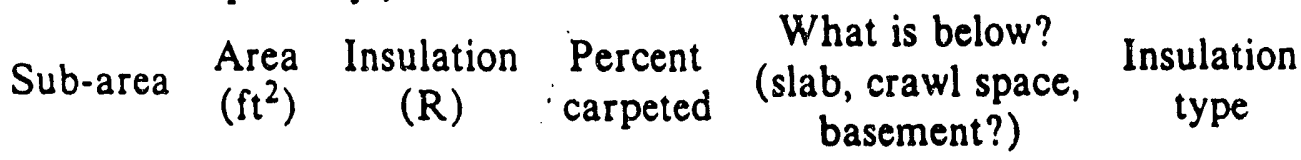

\begin{tabular}{|c|c|c|c|c|}
\hline $\begin{array}{l}\text { 1. } 2172 \mathrm{ft}^{2} \\
\text { 2. } \\
\text { 3. } \\
\end{array}$ & - & $75 \%$ & Slab & -- \\
\hline
\end{tabular}

VII. Windows and glass doors

\begin{tabular}{|c|c|c|}
\hline & $\begin{array}{l}\text { Single-pane } \\
\text { area }\end{array}$ & $\begin{array}{c}\text { Double-pane } \\
\text { area }\end{array}$ \\
\hline
\end{tabular}

Front of

house

$90 \mathrm{ft}^{2}$

$90 \mathrm{ft}^{2}$

(2)

Left side

$84 \mathrm{ft}^{2}$

$84 \mathrm{ft}^{2}$

of house

Right side

of house

$36 \mathrm{ft}^{2}$

$36 \mathrm{ft}^{2}$

Back of

house

$135 \mathrm{ft}^{2}$

$135 \mathrm{ft}^{2}$

VIII. Doors (non-glass)

$\begin{array}{cccc}\text { Orientation } & \begin{array}{c}\text { Regular } \\ \text { door } \\ \text { area }\end{array} & \begin{array}{c}\text { Regular door with } \\ \text { storm door or } \\ \text { thermally insulated } \\ \text { door area }\end{array} & \begin{array}{c}\text { Total } \\ \text { area }\end{array} \\ & & \end{array}$

\begin{tabular}{lcc}
\hline Front of house & 36 & 36 \\
Left side of house & & \\
$\begin{array}{l}\text { Right side of house } \\
\text { Back of house } \\
\text { Totals }\end{array}$ & 36 & 36 \\
\hline
\end{tabular}


IX. Heating and cooling systems

1. Main heating system type:

Gas furnace

Gas boiler (steam)

Gas boiler (hot water) Oil furnace

Oil boiler (steam) Oil boiler (hot water)

Elec. furnace Elec. ceiling Elec. baseboard

Central heat pump $\mathrm{X}$ Window heat pump

Other (specify)

If oil fired, has a flame retention head burner been installed?

Yes No

If oil boiler, is it wet base or dry base

Approximate age of system 18 years

Location of unit within house Garage

Is this a converted coal unit? Yes No

Heating value of fuel: Gas

Oil

Nameplate information, as available:

Manufacturer Florida Heat Pump

Model 301

Input capacity

Output capacity 36,000 Btu/hr

SEER $<6$

Is the pilot light turned off in the spring? Yes _ No _ N/A _ Energy efficiency devices present:

Vent damper IID Other (specify)

2. Distribution system

No Juctwork or piping Hydronic (gravity)

Hydronic (pumped) Forced-air Btu/hr

Baseboard Gravity system (no fans)

Other (specify) 
If ductwork or piping present:

Total length of ducts or piping $180 \mathrm{ft}$

Percentage of ducts or piping in unconditioned (non-living) spaces $100 \%$

Are ducts or piping in unconditioned spaces insulated?

Yes $\mathrm{X}$ No If yes, approximate R-value 5

3. Main cooling system type:

None Window $\mathrm{A} / \mathrm{C}$ Central A/C

Evaporative cooler Central heat pump $\mathrm{x}$

Window heat pump Other (specify)

Approximate age of system 18 years

Nameplate information, as available

Manufacturer Florida Heat Pump

Model 301

Input capacity $\mathrm{Btu} / \mathrm{hr}$

Output capacity $\quad 36,000$ But $/ \mathrm{hr}$

SEER

Location(s) within house Garage

4. Secondary heating systems:

No. fireplaces 1

No. fireplace inserts

No. wood stoves

No. electric space heaters

No. fossil fuel space heaters

Other (specify)

How often are these used?

5 times per year

5. Thermostat:

None Normal $\mathrm{X}$

Clock Other (specify)

Location within house Kitchen, above air return 
$X$. Significant external loads:

Irrigation pumps

Welder

Outside lights Other (specify)

Frequency of use

XI. Experimental retrofit installation:

Description A/C upgrade (SEER 6 to 13.2) Florida Heat Pump SE 038-1

Attic Insulation $<\mathrm{R} 7$ to $\mathrm{R} 30$

Actual installation cost $\$ \frac{2860.00(\mathrm{~A} / \mathrm{C})}{633.00}$

633.00 (Insulation) 


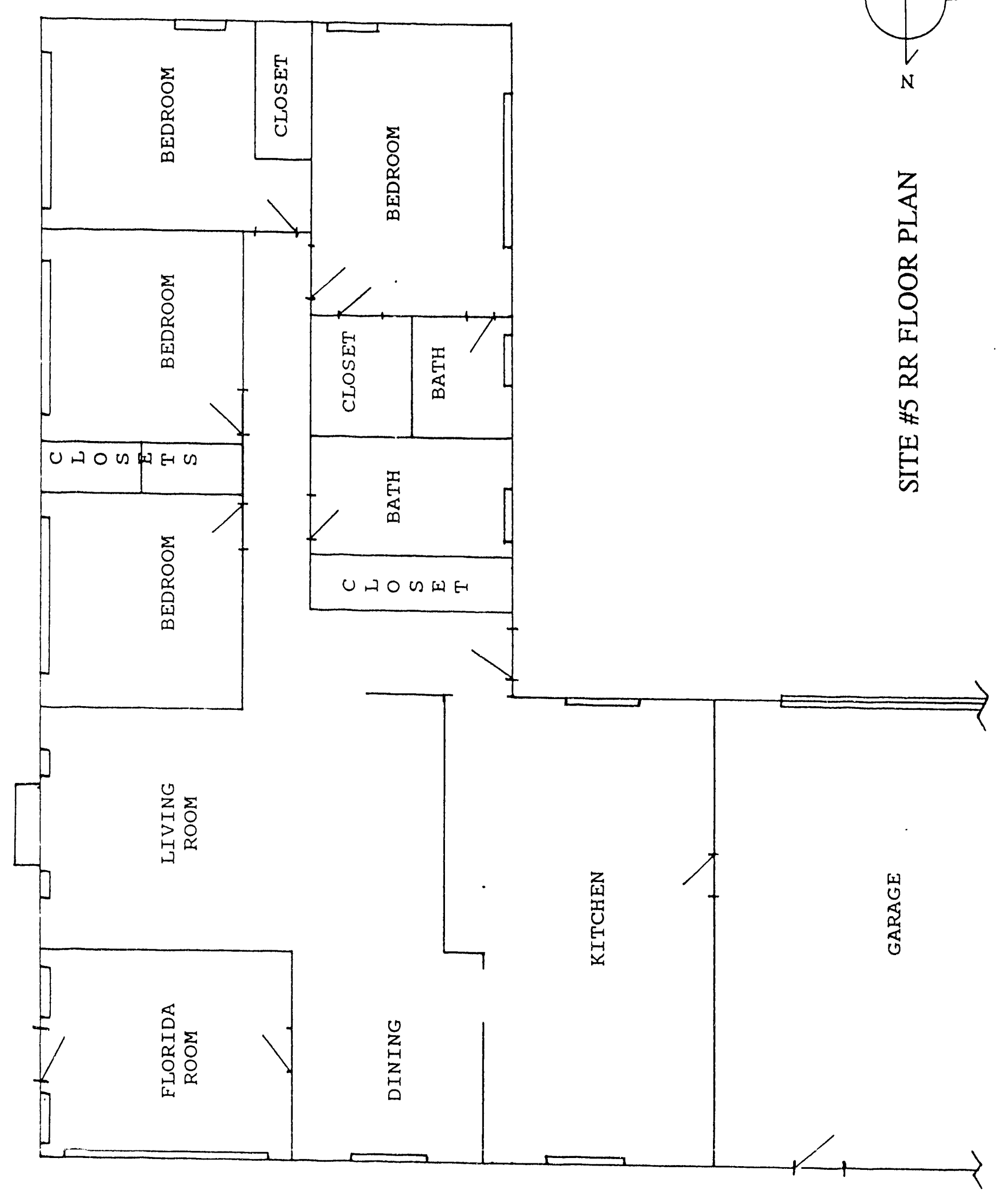



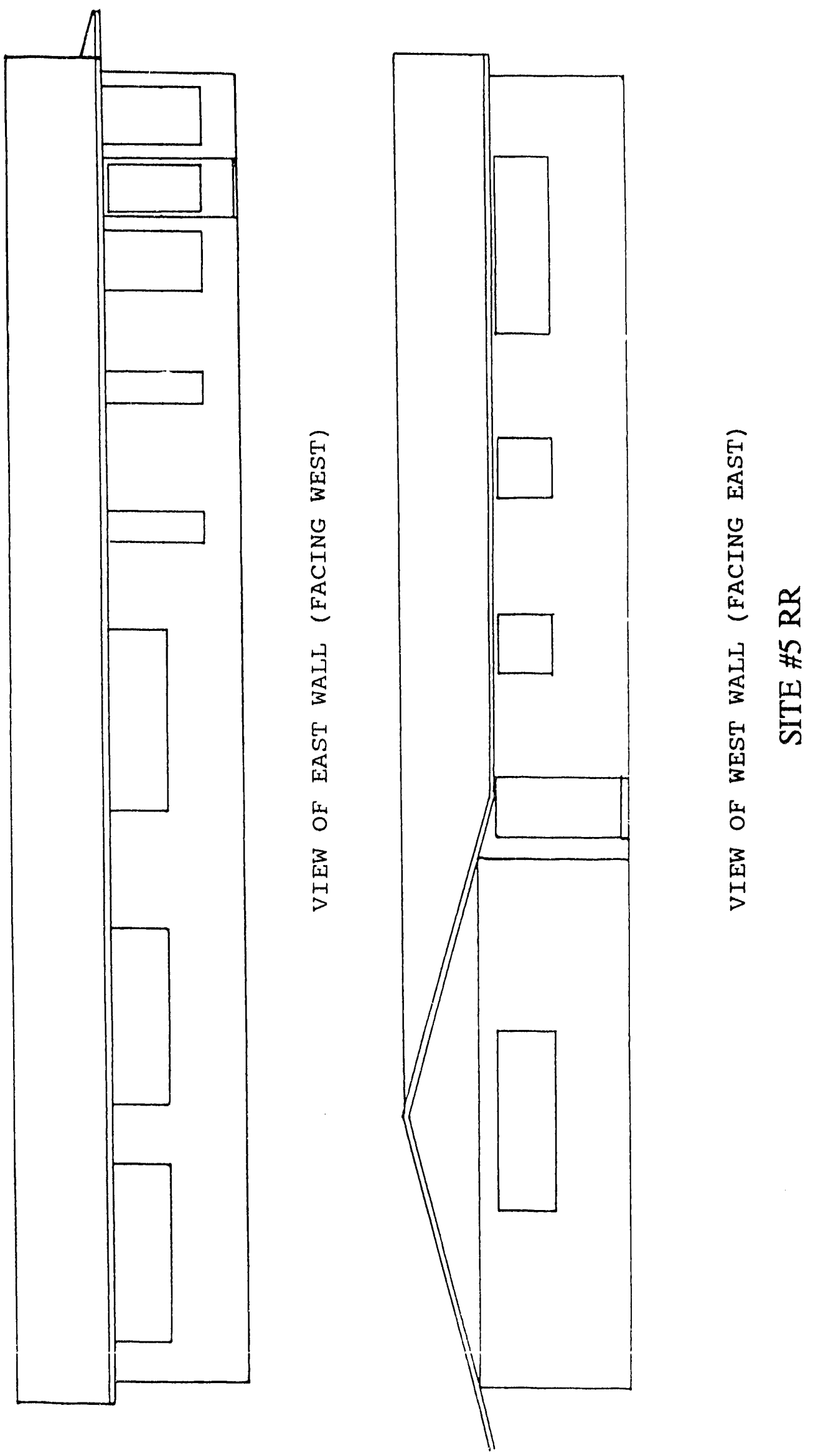

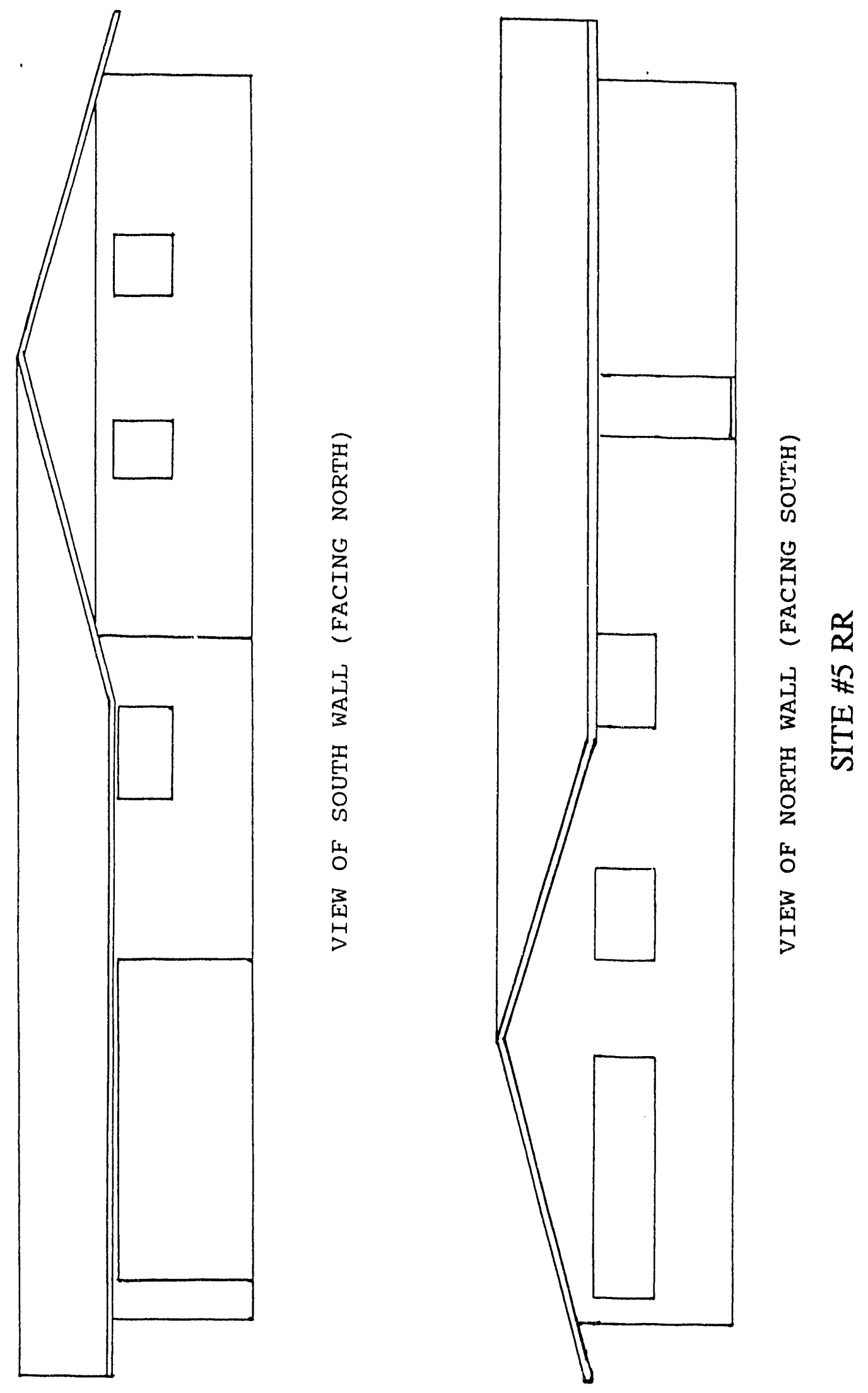
INFILTRATION RATE EVALUATION FACTORS

\begin{tabular}{|c|c|c|c|}
\hline $\begin{array}{l}\text { Building } \\
\text { component }\end{array}$ & Good & Average & Poor \\
\hline $\begin{array}{l}\text { Windows } \\
\text { and } \\
\text { doors }\end{array}$ & $\begin{array}{l}\text { Window and door frames } \\
\text { caulked. Window and door } \\
\text { sashes well fitting and weather- } \\
\text { stripped or storm windows and } \\
\text { doors with good fit. }\end{array}$ & $\begin{array}{l}\text { Window and door frames } \\
\text { caulked or window and door } \\
\text { sashes weatherstripped or } \\
\text { poorly fitting storm doors and } \\
\text { windows. }\end{array}$ & $\begin{array}{l}\text { No caulking on window and } \\
\text { door frames. No weather- } \\
\text { stripping. No storm doors or } \\
\text { windows. }\end{array}$ \\
\hline $\begin{array}{l}\text { Walls and } \\
\text { electrical } \\
\text { outlets }\end{array}$ & $\begin{array}{l}\text { Ceiling and foor joints and } \\
\text { corners well sealed. Elec- } \\
\text { trical outlets with gaskets. No } \\
\text { holes around plumbing penetra- } \\
\text { tions. }\end{array}$ & $\begin{array}{l}\text { Some cracks in ceiling and } \\
\text { floor joints and corners. No } \\
\text { gaskets on electrical outlets. } \\
\text { Fewer than three plumbing } \\
\text { penetrations with holes } \\
\text { around them. }\end{array}$ & $\begin{array}{l}\text { Many cracks in ceiling, } \\
\text { loor joints, and corners. No } \\
\text { gaskets on electrical outlets. } \\
\text { Three or more plumbing } \\
\text { penetrations with holes } \\
\text { around them. }\end{array}$ \\
\hline $\begin{array}{l}\text { Altic } \\
\text { Moor } \\
\text { (ceiling) }\end{array}$ & $\begin{array}{l}\text { No cracks in attic noor. No } \\
\text { air shafts around flues. No } \\
\text { holes around ducts, pipes, or } \\
\text { wiring penetrating attic noor. } \\
\text { No recessed light fixtures. No } \\
\text { trap door or weatherstripped } \\
\text { trap door to attic. }\end{array}$ & $\begin{array}{l}\text { Some cracks in attic floor. } \\
\text { No air shafts around flues. } \\
\text { Some holes around ducts, pipes, } \\
\text { or wiring penetrating attic floor. } \\
\text { Fewer than three recessed light } \\
\text { fixtures. Unweatherstripped } \\
\text { trap door to attic. }\end{array}$ & $\begin{array}{l}\text { Many cracks in attic floor. } \\
\text { Air shafts around llues. } \\
\text { Holes around ducts, pipes, or } \\
\text { wiring penetrating attic floor. } \\
\text { More than three recessed light } \\
\text { fixtures. Uncovered attic } \\
\text { access. }\end{array}$ \\
\hline $\begin{array}{l}\text { Heating } \\
\text { system } \\
\text { and water } \\
\text { heater }\end{array}$ & $\begin{array}{l}\text { Xoth furnace and water } \\
\text { heater electric. If fossil fuel } \\
\text { fired, both in unconditioned } \\
\text { space. }\end{array}$ & $\begin{array}{l}\text { One fossil fuel-fired unit in } \\
\text { living space with vent } \\
\text { damper. The other in uncondi- } \\
\text { tioned space. }\end{array}$ & $\begin{array}{l}\text { At least one fossil fuel-fired } \\
\text { unit in living space without } \\
\text { vent damper. }\end{array}$ \\
\hline $\begin{array}{l}\text { Fireplace } \\
\text { or wood } \\
\text { stove }\end{array}$ & $\begin{array}{l}\text { XX Sealed combustion wood } \\
\text { stove or fireplace with well- } \\
\text { fitting damper and glass doors } \\
\text { or no fireplace. }\end{array}$ & $\begin{array}{l}\square \text { Poorly sealed wood siove } \\
\text { or fireplace with either well- } \\
\text { fitting damper or glass doors. }\end{array}$ & $\begin{array}{l}\text { Both a wood stove and fire- } \\
\text { place or a fireplace with } \\
\text { poorly fitting damper and no } \\
\text { glass doors. }\end{array}$ \\
\hline $\begin{array}{l}\text { Duciwork } \\
\text { and } \\
\text { noor }\end{array}$ & $\begin{array}{l}\text { No ductwork and few noor } \\
\text { penetrations or all ductwork } \\
\text { in conditioned space and } \\
\text { no floor penetration. }\end{array}$ & $\begin{array}{l}0 \text { Ductwork in conditioned } \\
\text { basement and few floor } \\
\text { penetrations. }\end{array}$ & 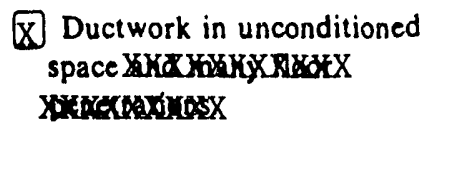 \\
\hline $\begin{array}{l}\text { Vents in } \\
\text { conditioned } \\
\text { space }\end{array}$ & $\begin{array}{l}\text { No undampered vents and } \\
\text { fewer than three dampered } \\
\text { vents. }\end{array}$ & $\begin{array}{l}\square \text { Fewer than three undampered } \\
\text { vents or at least three } \\
\text { dampered vents. }\end{array}$ & $\begin{array}{l}\square \text { More than three undampered } \\
\text { yents. }\end{array}$ \\
\hline Lifestyle & $\begin{array}{l}\square \text { Fewer than six entrances and } \\
\text { exits per day. }\end{array}$ & $\begin{array}{l}\text { Xix to thirteen entrances } \\
\text { and exits per day. }\end{array}$ & $\begin{array}{l}\text { More than thirteen } \\
\text { entrances and exits per day. }\end{array}$ \\
\hline
\end{tabular}




\section{POST-RETROFIT AUDIT DATA FORM}

Retrofit program name Monitoring Conservative Retrofits in Single Family Bldgs Sponsoring organization(s) DOE/FPL/State of FL

Experimental site designation

$\mathrm{RR}$ (非)

Interviewer name

CSR

Date of interview

$9 / 15 / 92$

I. General information from householder

1. Name R.E. Rainsberger

2. Street address

City State

3. Phone number

4. Own Rent

5. If householder different from that of "Pre-Retrofit Audit," or if tenure has changed, record below:

Change in householder Date of change

Change in tenure Date of change

6. Number of occupants, by age group:

\begin{tabular}{lc}
\multicolumn{1}{c}{ Age group } & Number \\
\hline Preschool & \\
School age & \\
Adult, but not retired & \\
Retired &
\end{tabular}

How many are generally home all day?

\begin{tabular}{ll} 
Weekday & 0 \\
Weekend & 2 \\
\hline
\end{tabular}

II. Significant changes since pre-retrofit audit NONE

(The remaining questions refer to the time since the energy-saving improvements were made to the house).

1. Have there been any important changes or additions io the house, such as (check appropriate): No

Rooms added Siding added

Garage/carport added Exterior paint 
Porch added

New roof

Other (specify)

2. Othe" than changes of this program, has the main heating or cooling system been changed, such as (check appropriate): None

New furnace/boiler

New air conditioner

New heat pump

Modified furnace/boiler (specify)

Other (specify)

3. Are the same number of rooms still heated and cooled? (If not, specify) Yes

4. Have any space heaters been aodded/subtracted? (Fireplaces, wood stoves, electric, fossil fuel, etc.) Yes No $\mathrm{X}$

If so, specify

Has the use of these heaters changed during this last winter?

Used more this winter _ Less _ Same $\mathrm{x}$ Do not know -

5. Has the hot water heating system changed? Yes _ No $\underline{x}$ If so, specify

6. Have any major appliances been added/subtracted? (Stoves, ovens, washers, dryers, etc.) No

If so, specify

7. Is there: An attic fan No Whole house fan No

If so, has the use of these fans changed during this last summer?

Used more this summer _ Less _ Same _ Do not know

8. Other than changes of this program, have any other energy-saving changes been installed this past year? Yes _ No $\mathrm{X}$

If yes, specify:

Ceiling insulation added

Wall insulation added

Storm windows added Weatherstripping

Storm doors added Caulking

Other (specify) Hot water flow restrictors Clock thermostat 
9. Have you or any family members taken up any new hobbies during the past year that require the use of large electrical or gas equipment?

Yes _ No

If so, specify 


\section{PRE-RETROFIT AUDIT DATA FORM}

Retrofit program name Monitoring Conservative Retrofits in Single Family Bldgs Sponsoring organization(s) DOE/FPL/State of FL

Experimental site designation $\mathrm{BC}(\# 6)$

Interviewer name CSR Date of interview $7 / 28 / 91$

I. General information from householder

1. Name Bernard J. Cohn

2. Street address 154 Abalone Road

City Palm Bay State FL 32907

3. Phone number 951-7702

4. Number of occupants, by age group:

\begin{tabular}{ll}
\multicolumn{1}{c}{ Age group } & Number \\
\hline Preschool & - \\
School age & -1 \\
Adult, but not retired & -2 \\
Retired &
\end{tabular}

How many are generally home all day?

Weekday

Weekend

$\frac{0}{3}$

5. Approximate age of house 8 years

6. Names of utility distributors:

Electric EPL

Gas

Oil

(Note: Auditor should have homeowner sign a release authorizing release by these utilities of fuel bills for preceding two years.)

7. Ow'n $\mathrm{X}$ Rent

8. Length of time at this address years

II. General description of house

1. Type: S-F detached $\mathrm{X}$ S-F attached Mobile home Other (specify) 
2. Style: One-story $\mathrm{X}$ Two-story Three-story Split-level One-and-a-half-story

3. Foundation: Basement Crawl space Slab $\mathrm{X}$ Other (specify)

4. Exterior colors: Roof Brown Walls Brick

5. Layout, dimensions, orientation: Use attached House Sketch Sheet.

6. Percentagè of each floor that is conditioned (heated or cooled):

\begin{tabular}{lcc} 
Floor of house & $\begin{array}{c}\text { Percentage } \\
\text { heated }\end{array}$ & $\begin{array}{c}\text { Percentage } \\
\text { cooled }\end{array}$ \\
\hline $\begin{array}{l}\text { Basement } \\
\text { First floor }\end{array}$ & $100 \%$ & NA \\
Second floor & & \\
Third floor & & \\
Other (specify) & &
\end{tabular}

7. Infiltration: Use attached Infiltration Rate Evaluation Factors sheet.

8. Kooms typically closed off None

III. Major appliances

1. Domestic hot water

Type: Elec. resistance Heat pump hot water

Gas Solar

Other (specify)

Nameplate infor. ation, as available:

Manufacturer Bradford-White

Model $\quad M=3-580 \quad R 55$

Tank capacity (gallons) 80 gal

SEER

Tank insulation ( $R$-value)

Location Utility room

Hot water temperature (measured with thermometer) see data ${ }^{\circ} \mathrm{F}$

2. Solar devices

None $\mathrm{X}$ Domestic hot water Swimming pool heater

Space heating Other (specify) 
3. Other major appliances, by fuel type:

\begin{tabular}{|c|c|c|c|c|}
\hline Appliance & None & Electric & Gas & $\begin{array}{l}\text { Other } \\
\text { (specify) }\end{array}$ \\
\hline Cooking range/oven & & $\mathrm{x}$ & & \\
\hline Clothes washer & & $\mathrm{X}$ & & \\
\hline Clothes dryer & & $\mathrm{X}$ & & \\
\hline $\begin{array}{l}\text { Refrigerator/freezer } \\
\text { Separate freezer }\end{array}$ & & $\mathrm{X}$ & & \\
\hline Conventional oven & & $\mathrm{x}$ & & \\
\hline Microwave oven & & $\mathrm{X}$ & & \\
\hline Dishwasher & & $\mathrm{X}$ & & \\
\hline Whole house fan & & & & \\
\hline Attic fan & & & & \\
\hline Water bed heater & & & & \\
\hline Other & & & & \\
\hline
\end{tabular}

IV. Ceiling area and insulation

(If different parts of the ceiling have different insulation, treat each of these sub-areas separately.)

\begin{tabular}{|c|c|c|c|}
\hline Sub-area & $\begin{array}{l}\text { Area } \\
\left(f t^{2}\right)\end{array}$ & $\begin{array}{l}\text { Insulation } \\
\text { (R) }\end{array}$ & $\begin{array}{c}\text { Insulation } \\
\text { type }\end{array}$ \\
\hline $\begin{array}{l}1 . \\
2 . \\
3\end{array}$ & 1256 & 19 & batts \\
\hline
\end{tabular}

V. Wall area and insulation

(If different parts of wall have different materials, treat each of these sub-areas separately.)

Exterior wall construction: (1)Wood; 2) Brick (3) Stone; (4) Stucco;

(5) Concrete block; (6) Metal;

(7) Other (specify)

\begin{tabular}{ccccc} 
Sub-area & $\begin{array}{c}\text { Area } \\
\left(\mathrm{ft}^{2}\right)\end{array}$ & $\begin{array}{c}\text { Insulation } \\
(\mathrm{R})\end{array}$ & $\begin{array}{c}\text { Exterior wall } \\
\text { construction }\end{array}$ & $\begin{array}{c}\text { Insulation } \\
\text { type }\end{array}$ \\
\hline $\begin{array}{c}1 . \\
2 .\end{array}$ & $1300 \frac{1}{2}$ & 11 & Brick & batts \\
3. & & & & \\
Total & $1300 \frac{1}{2}\left(\mathrm{ft}^{2}\right)$ & & &
\end{tabular}


VI. Floor area and insulation (bottom floor only)

(If different parts of floor have different materials, treat each of these sub-areas separately.)

\begin{tabular}{cccccc} 
Sub-area & $\begin{array}{c}\text { Area } \\
\left(\mathrm{ft}^{2}\right)\end{array}$ & $\begin{array}{c}\text { Insulation } \\
(\mathrm{R})\end{array}$ & $\begin{array}{c}\text { Percent } \\
\text { carpeted }\end{array}$ & $\begin{array}{c}\text { What is below? } \\
\text { (slab, crawl space, } \\
\text { basement?) }\end{array}$ & $\begin{array}{c}\text { Insulation } \\
\text { type }\end{array}$ \\
\hline $\begin{array}{c}1 . \\
2 . \\
3 .\end{array}$ & 1256 & & $60 \%$ & Slab & - \\
Total $1256\left(\mathrm{ft}^{2}\right)$ & & & &
\end{tabular}

V'II. W'indow's and glass doors

\begin{tabular}{|c|c|c|c|}
\hline Orientation & $\begin{array}{l}\text { Single-pane } \\
\text { area }\end{array}$ & $\begin{array}{c}\text { Double-pane } \\
\text { area }\end{array}$ & $\begin{array}{c}\text { Total area } \\
\text { oriented }\end{array}$ \\
\hline $\begin{array}{l}\text { Front of } \\
\text { house }\end{array}$ & $45 \mathrm{ft}^{2}$ & & $45 f t^{2}$ \\
\hline
\end{tabular}

Left side $\quad 54 \mathrm{ft}^{2} \quad 54 \mathrm{ft}^{2}$

of house

of house

$54 \mathrm{ft}^{2}$

$54 \mathrm{ft}^{2}$

Of house

Back of house

$$
18 \mathrm{ft}^{2}
$$

$18 \mathrm{ft}^{2}$

house

VIII. Doors (non-glass)

\begin{tabular}{lccc}
\multicolumn{1}{c}{ Orientation } & $\begin{array}{c}\text { Regular } \\
\text { door } \\
\text { area }\end{array}$ & $\begin{array}{c}\text { Regular door with } \\
\text { storm door or } \\
\text { thermally insulated } \\
\text { door area }\end{array}$ & $\begin{array}{c}\text { Total } \\
\text { area }\end{array}$ \\
\hline $\begin{array}{l}\text { Front of house } \\
\text { Left side of house }\end{array}$ & 39.00 & & 39.00 \\
$\begin{array}{l}\text { Right de of house } \\
\text { Back of house }\end{array}$ & 16.25 & & \\
$\quad$ Totals & 55.25 & & 16.25 \\
\hline
\end{tabular}


IX. Heating and cooling systems

1. Main heating system type:

Gas furnace

Gas boiler (steam)

Gas boiler (hot water)

Oil furnace

Oil boiler (steam) Oil boiler (hot water)

Elec. furnace

Elec. ceiling Elec. baseboard

Central heat pump

Window heat pump

Other (specify) Resistance coils in $\mathrm{A} / \mathrm{H}$

If oil fired, has a flame retention head burner been installed?

Yes No

If oil boiler. is it wet base

Approximate age of system or dry base

Location of unit within house years

Is this a converted coal unit? Yes closet

Heating value of fuel: $\mathrm{Gas}$

Oil

Nameplate information, as available:

Manufacturer

Model

Input capacity Btu/hr

Output capacity Btu/hr

SEER

Is the pilot light turned off in the spring? Yes No N/A $\underline{x}$ Energy efficiency devices present:

Vent damper IID Other (specify)

2. Distribution system

No ductwork or piping Hydronic (pumped) Hydronic (gravity)

Baseboard Forced-air $\mathrm{x}$ Other (specify) 
If ductwork or piping present:

Total length of ducts or piping 80 $\mathrm{ft}$

Percentage of ducts or piping in unconditioned (non-living) spaces $100 \%$ Are ducts or piping in unconditioned spaces insulated?

Yes $\quad \mathrm{X}$ No If yes, approximate R-value -5

3. Main cooling system type:

None Window $\mathrm{A} / \mathrm{C}$ Central A/C

Evaporative cooler Central heat pump

Window heat pump Other (specify)

Approximate age of system years

Nameplate information, as available

Manuiacturer Janitrol

Model

Input capacity $\mathrm{Btu} / \mathrm{hr}$

Output capacity' But $/ \mathrm{hr}$

SEER estimate approximately 6

Location(s) within house Closet

4. Secondary heating systems: None

No. fireplaces

No. fireplac : inserts

No. wood stoves

No. electric space heaters

No. fossil fuel space heaters

Other (specify)

How often are these used?

5. Thermostat:

None Normal $x$

Clock Other (specify)

Location within house living room, near air return 
$X$. Significant external loads:

Irrigation pumps

Welder

Outside lights Other (specify)

Pool Pump

Frequency of use 6 hrs per day

XI. Experimental retrofit installation:

Description

Lennox HP-22-311-2P compressor

$\mathrm{CB}-18-41-3 \mathrm{P}$

$\mathrm{A} / \mathrm{H}$ unit

Actual installation cost $\$ 2800.00$ 

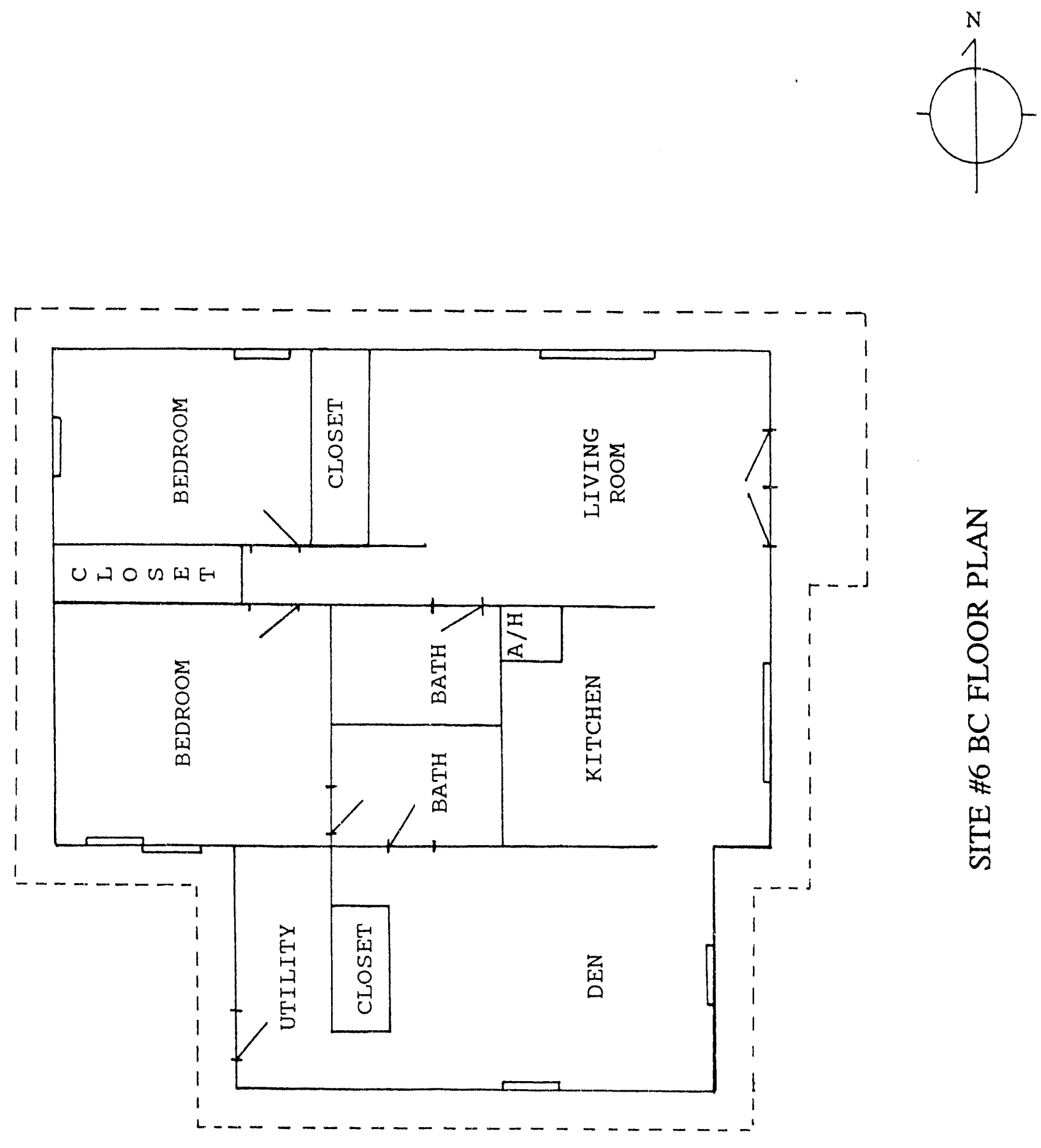

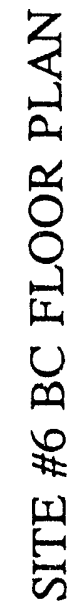



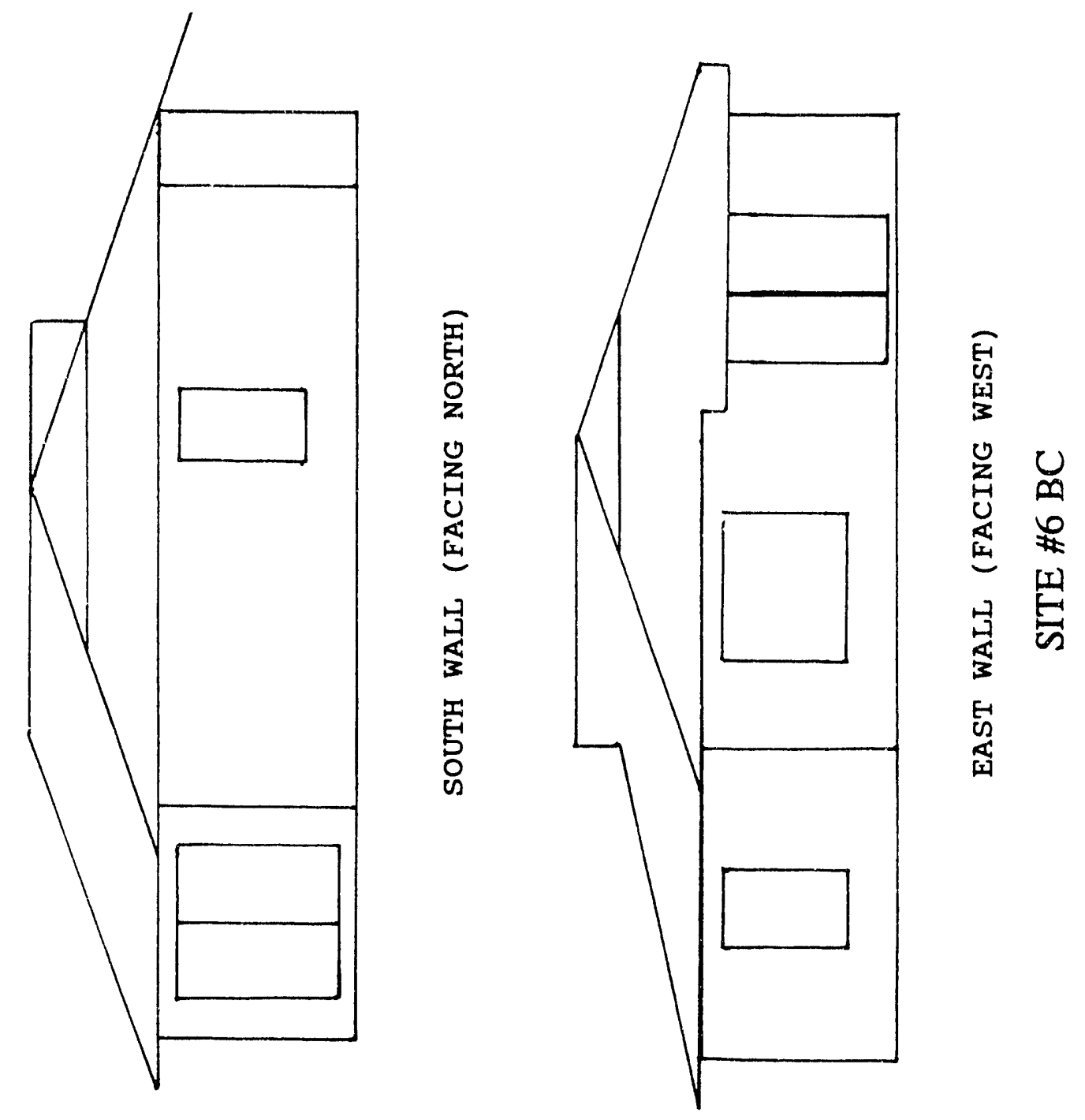


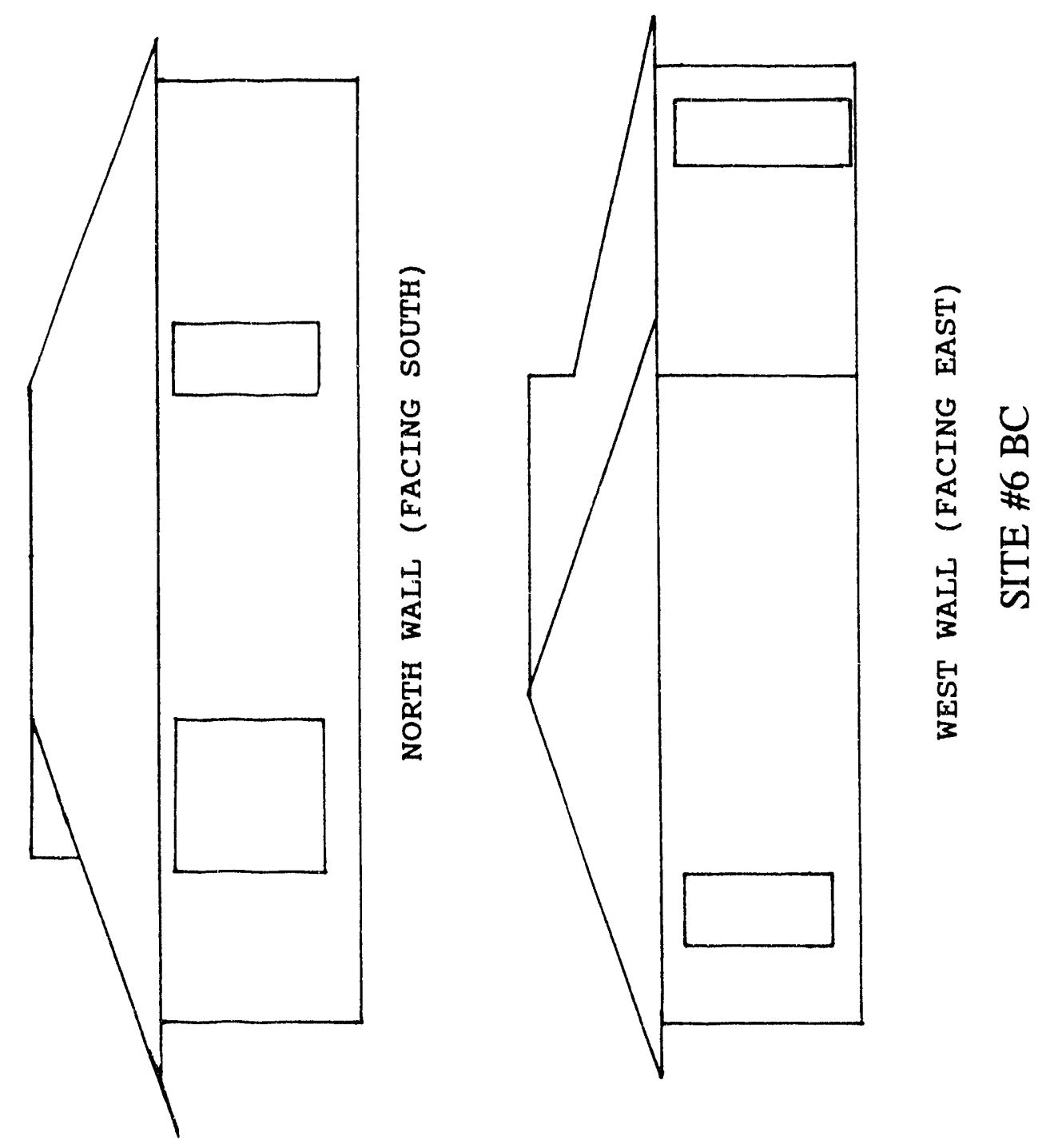

$\bar{\equiv}$

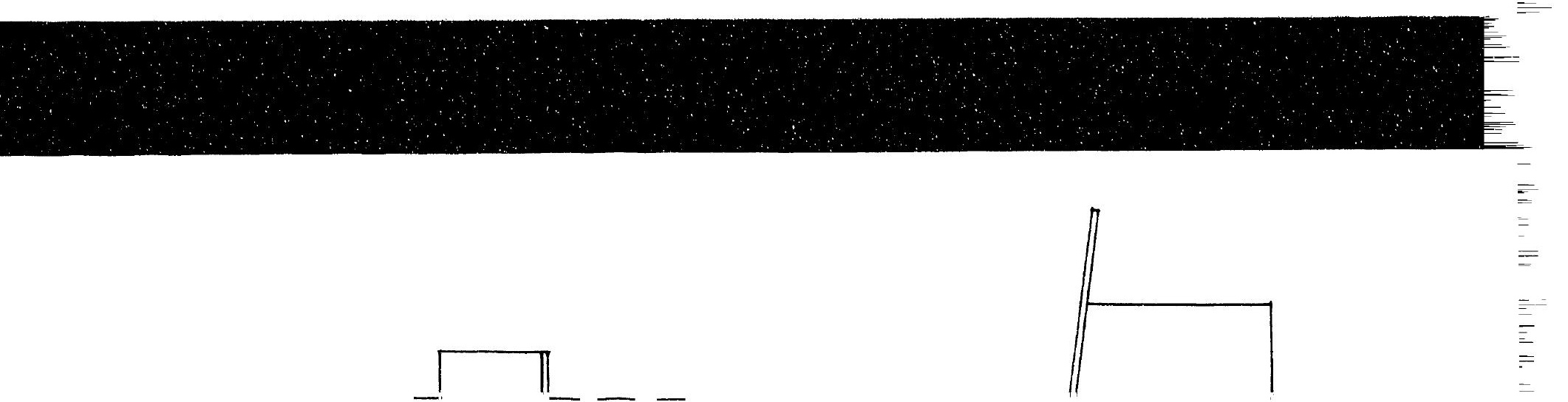


INFILTRATION RATE EVALUATION FACTORS

\begin{tabular}{|c|c|c|c|}
\hline $\begin{array}{l}\text { Building } \\
\text { component }\end{array}$ & Good & Average & Poor \\
\hline $\begin{array}{l}\text { Winonus } \\
\text { and } \\
\text { doors }\end{array}$ & $\begin{array}{l}\text { Xindow and door frames } \\
\text { caulked. Window and door } \\
\text { sashes well fitting and weather- } \\
\text { stripped or storm windows and } \\
\text { doors with good fit. }\end{array}$ & $\begin{array}{l}\text { Window and door frames } \\
\text { caulked or window and door } \\
\text { sashes weatherstripped or } \\
\text { poorly fitting storm doors and } \\
\text { windows. }\end{array}$ & $\begin{array}{l}\text { No caulking on window and } \\
\text { door frames. No weather- } \\
\text { stripping. No storm doors or } \\
\text { windows. }\end{array}$ \\
\hline $\begin{array}{l}\text { Walls and } \\
\text { electrical } \\
\text { outlets }\end{array}$ & $\begin{array}{l}\text { X Ceiling and floor joints and } \\
\text { corners well sealed. Elec- } \\
\text { trical outlets with gaskets. No } \\
\text { holes around plumbing penetra- } \\
\text { tions. }\end{array}$ & $\begin{array}{l}\text { Some cracks in ceiling and } \\
\text { floor joints and corners. No } \\
\text { gaskets on electrical outlets. } \\
\text { Fewer than three plumbing } \\
\text { penetrations with holes } \\
\text { around them. }\end{array}$ & $\begin{array}{l}\text { Many cracks in ceiling, } \\
\text { floor joints, and corners. No } \\
\text { gaskets on electrical outlets. } \\
\text { Three or more plumbing } \\
\text { penetrations with holes } \\
\text { around them. }\end{array}$ \\
\hline $\begin{array}{l}\text { Attic } \\
\text { noor } \\
\text { (ceiling) }\end{array}$ & $\begin{array}{l}\text { X No cracks in attic noor. No } \\
\text { air shafts around nues. No } \\
\text { holes around ducts, pipes, or } \\
\text { wiring penetrating attic ncor. } \\
\text { No recessed light fixtures. No } \\
\text { trap door or weatherstripped } \\
\text { trap door to attic }\end{array}$ & $\begin{array}{l}\text { Some cracks in attic floor. } \\
\text { No air shafts around flues. } \\
\text { Some holes around ducts, pipes, } \\
\text { or wiring penetrating attic floor. } \\
\text { Fewer than three recessed light } \\
\text { fixtures. Unweatherstripped } \\
\text { trap door to attic. }\end{array}$ & $\begin{array}{l}\text { Many cracks in attic floor. } \\
\text { Air shafts around flues. } \\
\text { Holes around ducts, pipes, or } \\
\text { wiring penetrating attic floor. } \\
\text { More than three recessed ligh: } \\
\text { fixtures. Uncovered attic } \\
\text { access. }\end{array}$ \\
\hline $\begin{array}{l}\text { Heating } \\
\text { system } \\
\text { and water } \\
\text { heater }\end{array}$ & $\begin{array}{l}\text { Xoth furnace and water } \\
\text { heater electric If fossil fuel } \\
\text { fired. both in unconditioned } \\
\text { space. }\end{array}$ & $\begin{array}{l}\text { One fossil fuel-fired unit in } \\
\text { living space with vent } \\
\text { damper. The other in uncondi- } \\
\text { tioned space. }\end{array}$ & $\begin{array}{l}\text { At least one fossil fuel-fired } \\
\text { unit in living space without } \\
\text { vent damper. }\end{array}$ \\
\hline $\begin{array}{l}\text { Fireplace } \\
\text { or nowi } \\
\text { stove }\end{array}$ & $\begin{array}{l}\text { X Sealed combustion wood } \\
\text { stove or fireplace with well. } \\
\text { fitting damper and glass doors } \\
\text { or no fireplace. }\end{array}$ & $\begin{array}{l}0 \text { Poorly sealed wood stove } \\
\text { or fireplace with either well. } \\
\text { fitting damper or glass doors. }\end{array}$ & $\begin{array}{l}\text { Both a wood stove and fire- } \\
\text { place or a fireplace with } \\
\text { poorly fitting damper and no } \\
\text { glass doors. }\end{array}$ \\
\hline $\begin{array}{l}\text { Ductuori } \\
\text { and } \\
\text { noor }\end{array}$ & $\begin{array}{l}\text { No ductwork and few floor } \\
\text { penetrations or all ductwork } \\
\text { in conditioned space and } \\
\text { no Moor penetration. }\end{array}$ & $\begin{array}{l}\text { Ductwork in conditioned } \\
\text { basement and few floor } \\
\text { penetrations. }\end{array}$ & $\begin{array}{l}\text { Q Ductwork in unconditioned } \\
\text { space XWd } \\
\text { phrothonsk }\end{array}$ \\
\hline $\begin{array}{l}\text { Vents in } \\
\text { conditioned } \\
\text { space }\end{array}$ & $\begin{array}{l}\text { XX No undampered vents and } \\
\text { fewer than three dampered } \\
\text { vents }\end{array}$ & $\begin{array}{l}\text { Fewer than three undampered } \\
\text { vents or at least three } \\
\text { dampered vents. }\end{array}$ & $\begin{array}{l}\text { More than three undampered } \\
\text { vents. }\end{array}$ \\
\hline Lifestyle & $\begin{array}{l}\sqrt{X X} \text { Fewer than six entrances and } \\
\text { exits per das. }\end{array}$ & $\begin{array}{l}\text { Six to thirteen entrances } \\
\text { and exits per day. }\end{array}$ & $\begin{array}{l}\text { More than thirteen } \\
\text { entrances and exits per day. }\end{array}$ \\
\hline
\end{tabular}




\section{POST-RETROFIT AUDIT DATA FORM}

Retrofit program name Monitoring Conservative Retrofits in Single Family Bldgs

Sponsoring organization(s) DOE/FPL/State of FL

Experimental site designation

$B C($ 非 $)$

Interviewer name

$\operatorname{CSR}$

Date of interview

$9 / 15 / 92$

1. General information from householder

1. Name Bernard J. Cohn

2. Street address

City

State

3. Phone number

4. Owin

Rent

5. If householder different from that of "Pre-Retrofit Audit," or if tenure has changed, record below:

Change in householder Date of change

Change in tenure Date of change

6. Number of occupants, by age group:

\begin{tabular}{ll}
\multicolumn{1}{c}{ Age group } & Number \\
\hline Preschool & \\
School age \\
Adult, but not retired \\
Retired
\end{tabular}

How many are generally home all day?

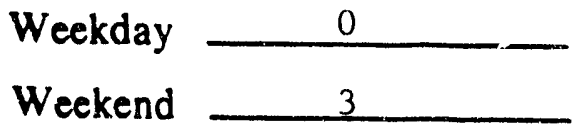

II. Significant changes since pre-retrofit audit NONE

(The remaining questions refer to the time since the energy-saving improvements were made to the house).

1. Have there been any important changes or additions to the house, such as (check appropriate): No

Rooms added Siding added

Garage/carport added Exterior paint 
Porch added New roof

Oiher (specify)

2. Other than changes of this program, has the main heating or cooling system been changed, such as (check appropriaie): No

New furnace/boiler

New air conditioner

New heat pump

Modified furnace/boiler (specify)

Other (specify)

3. Are the same number of rooms still heated and cooled? (If not, specify) Yes

4. Have any space heaters been added/subtracted? (Fireplaces, wood stoves, electric, fossil fuel, etc.) Yes_ No $\mathrm{X}$

If so, specify

Has the use of these heaters changed during this last winter?

Used more this winter _ Less _ Same _ Do not know

5. Has the hot water heating system changed? Yes _ No $X$

If so, specify

6. Have any major appliances been added/subtracted? (Stoves, ovens, washers, dryers, etc.) No

If so, specify

7. Is there: An attic fan $\mathrm{x}$ Whole house fan

If so, has the use of these fans changed during this last summer? No

Used more this summer _ Less _ Same _ Do not know

Not used

8. Other than changes of this program, have any other energy-saving changes been installed this past year? Yes No $\underline{X}$

If yes, specify:

Ceiling insulation added Weatherstripping

Wall insulation added Caulking

Storm windows added Hot water flow restrictors

Storm doors added Clock thermostat

Other (specify') 
9. Have you or any family members taken up any new hobbies during the past year that require the use of large electrical or gas equipment?

Yes No $\mathrm{X}$

If so, specify 


\section{PRE-RETROFIT AUDIT DATA FORM}

Retrofit program name Monitoring Conservative Retrofits in Single Family Bldgs Sponsoring organization(s) DOE/FPL/State of FL

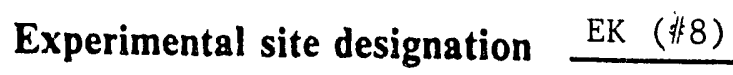

Interviewer name

$8 / 24 / 91$

I. General information from householder

1. Name Edward Kizak

2. Street address 216 Melbourne Avenue

City Indialantic State FL 32903

3. Phone number $723-8862$

4. Number of occupants, by age group:

\begin{tabular}{ll}
\multicolumn{1}{c}{ Age group } & Number \\
\hline Preschool & \\
School age & \\
Adult, but not retired & $-\frac{1}{1}$ \\
Retired &
\end{tabular}

How many are generally home all day?

Weekday $\frac{1}{2}$
Weekend

5. Approximate age of house 28 years

6. Names of utility distributors:

Electric FPL

Gas

Oil

(Note: Auditor should have homeowner sign a release authorizing release by these utilities of fuel bills for preceding two years.)

7. Own $\mathrm{x}$ Rent

8. Length of time at this address 28 years

II. General description of house

1. Type: S-F detached $x$ S-F attached

Mobile home Other (specify) 
2. Style: One-story $\mathrm{x}$ Two-story Three-story Split-level One-and-a-half-story

3. Foundation: Basement Crawl space Slab Other (specify)

4. Exterior colors: Rocf Grey Walls White

5. Layout, dimensions, orientation: Use attached House Sketch Sheet.

6. Percentage of each floor that is conditioned (heated or cooled):

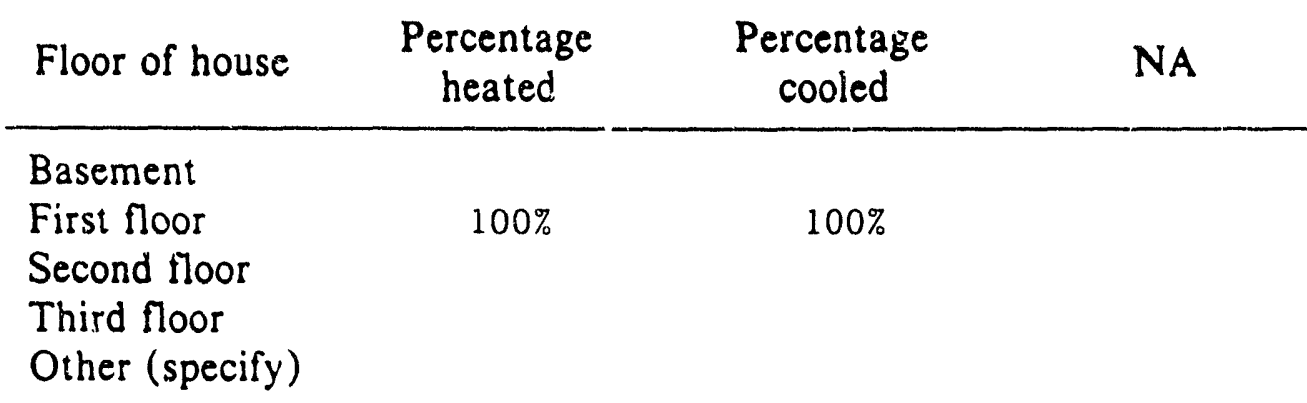

7. Infiltration: Use attached Infiltration Rate Evaluation Factors sheet.

8. Rooms typically closed off None

III. Majur appliances

1. Domestic hot water

Type: Elec. resistance $\mathrm{x}$ Heat pump hot water

Gas Solar

Other (specify)

Nameplate information, as available:

Manufacturer Reliance

Model 501

Tank capacity (gallons) $52 \mathrm{gal}$

SEER

Tank insulation ( $R$-value)

Location Hall closet

Hot water temperature (measured with thermometer) see data ${ }^{\circ} \mathrm{F}$

2. Solar devices

None $x$ Domestic hot water Suimming pool heater

Space heating Other (specify) 
3. Other major appliances, by fuel type:

\begin{tabular}{|c|c|c|c|c|c|}
\hline Appliance & None & Electric & Gas & $\begin{array}{l}\text { Other } \\
\text { (specify) }\end{array}$ & Location \\
\hline Cooking range/oven & & $\mathrm{x}$ & & & \multirow{12}{*}{ Garage } \\
\hline Clothes washer & & $\mathrm{x}$ & & & \\
\hline Clothes dryer & & $\mathrm{x}$ & & & \\
\hline Refrigerator/freezer & & $\mathrm{x}$ & & & \\
\hline Separate freezer & & $\mathrm{x}$ & & & \\
\hline Conventional oven & & $\mathrm{X}$ & & & \\
\hline Microwave oven & & $\mathrm{x}$ & & & \\
\hline Dishwasher & & $\mathrm{x}$ & & & \\
\hline Whole house fan & & & & & \\
\hline Attic fan & & & & & \\
\hline Water bed heater & & & & & \\
\hline Other & & & & & \\
\hline
\end{tabular}

IV. Ceiling area and insulation

(If different parts of the ceiling have different insulation, treat each of these sub-areas separately.)

\begin{tabular}{|c|c|c|c|}
\hline Sub-area & $\begin{array}{l}\text { Area } \\
\left(\mathrm{ft}^{2}\right)\end{array}$ & $\begin{array}{l}\text { Insulation } \\
\text { (R) }\end{array}$ & $\begin{array}{c}\text { Insulation } \\
\text { type }\end{array}$ \\
\hline & 650 & 19 & \\
\hline 2. 1 & $1180 \mathrm{ft}^{2}$ & 30 & blown \\
\hline $\begin{array}{l}3 . \\
\text { Total }\end{array}$ & $\begin{array}{l}645 \mathrm{ft}^{2} \\
\underline{2475}\end{array}$ & $\left.\mathrm{ft}^{2}\right)^{19}$ & batts \\
\hline
\end{tabular}

V. Wall area and insulation

(If different parts of wall have different materials, treat each of these sub-areas separafely.)

Exterior wall construction: (1)Wood; (2) Brick; (3) Stone; (4) Stucco;

(5) Concrete block (6) Metal;

(7) Other (specify)

\begin{tabular}{|c|c|c|c|}
\hline & $\begin{array}{l}\text { Are } \\
\left(\mathrm{ft}^{2}\right.\end{array}$ & $\begin{array}{l}\text { Insulation } \\
(R)\end{array}$ & $\begin{array}{l}\text { Exterior wall } \\
\text { construction }\end{array}$ \\
\hline
\end{tabular}

1. $3044 \frac{1}{2 t^{2}}$

2.

3.

Total $3044 \frac{1}{2}\left(\mathrm{ft}^{2}\right)$ 
VI. Floor area and insulation (bottom floor only)

(If different parts of floor have different materials, treat each of these sub-areas separately.)

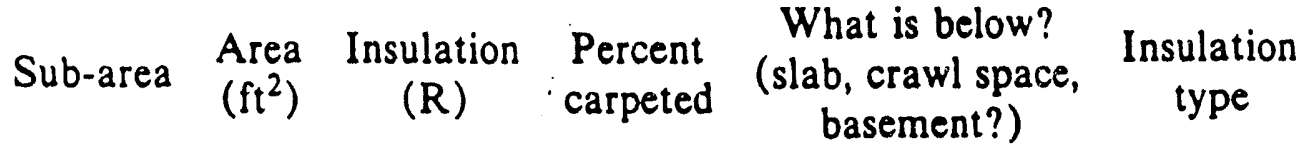

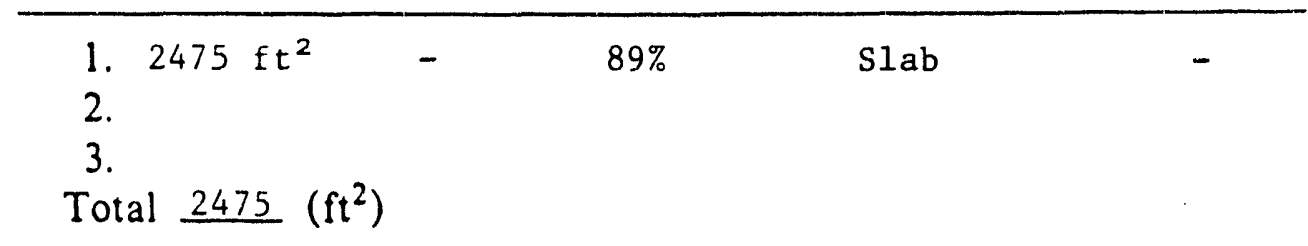

VII. Windows and glass doors

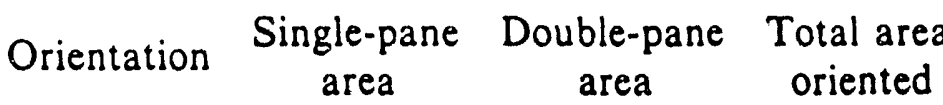

Front of $\quad 18 \mathrm{ft}^{2} \quad 18 \mathrm{ft}^{2}$

house

$18 \mathrm{ft}^{2}$

Left side $\quad 66 \mathrm{ft}^{2} \quad 66 \mathrm{ft}^{2}$

of house

Right side $\quad 58 \mathrm{ft}^{2} \quad 58 \mathrm{ft}^{2}$

of house

Back of

house

$45 \mathrm{ft}^{2} \quad 45 \mathrm{ft}^{2}$

VIII. Doors (non-glass)

\begin{tabular}{|c|c|c|c|c|}
\hline & Orientation & $\begin{array}{c}\text { Regular } \\
\text { door } \\
\text { area }\end{array}$ & $\begin{array}{l}\text { Regular door with } \\
\text { storm door or } \\
\text { thermally insulated } \\
\text { door area }\end{array}$ & $\begin{array}{l}\text { Total } \\
\text { area }\end{array}$ \\
\hline South & Front of house & - & - & - \\
\hline West & Left side of house & 19.5 & & 19.5 \\
\hline East & Right side of house & - & - & - \\
\hline North & $\begin{array}{c}\text { Back of house } \\
\text { Totals }\end{array}$ & $\overline{19.5}$ & - & $\begin{array}{l}- \\
19.5\end{array}$ \\
\hline
\end{tabular}


IX. Heating and cooling systems

1. Main heating system type:

Gas furnace Gas boiler (steam)

Gas boiler (hot water) Oil furnace

Oil boiler (steam) Oil boiler (hot water)

Elec. furnace Elec. ceiling Elec. baseboard

Central heat pump $\mathrm{x}$ Window heat pump

Other (specify)

If oil fired, has a flame retention head burner been installed?

Yes No

If oil boiler, is it wet base or dry base

Approximate age of system years

Location of unit within house

Is this a converted coal unit? Yes No

Heating value of fuel: Gas

$$
\text { Oil }
$$

Nameplate information, as available:

Manufacturer Florida Heat Pump

Model unknown

Input capacity

Output capacity 60,000 Btu/hr

SEER estimated approximately 6.1 $\mathrm{Btu} / \mathrm{hr}$

Is the pilot light turned off in the spring? Yes No N/A Energy efficiency devices present:

Vent damper IID Other (specify)

2. Distribution system

No ductwork or piping

Hydronic (pumped)

Baseboard Hydronic (gravity)

Other (specify) 
If ductwork or piping present:

Total length of ducts or piping $120 \mathrm{ft}$

Percentage of ducts or piping in unconditioned (non-living) spaces $100 \%$

Are ducts or piping in unconditioned spaces insulated?

Yes $\mathrm{X}$ No __ If yes, approximate R-value 4

3. Main cooling system type:

None Window $\mathrm{A} / \mathrm{C}$

Evaporative cooler Central heat pump $\mathrm{X}$

Window heat pump Other (specify)

Approximate age of system 12 years

Nameplate information, as available

Manufacturer Florida Heat Pump

Model unknown

Input capacity $\mathrm{Btu} / \mathrm{hr}$

Output capacity 60,000 $\mathrm{Btu} / \mathrm{hr}$

SEER

Location(s) within house

$\mathrm{A} / \mathrm{H}$ closet

4. Secondary heating systems:

No. fireplaces 1 not used

No. fireplace inserts

No. wood stoves

No. electric space heaters

No. fossil fuel space heaters

Other (specify)

How often are these used?

5. Thermostat:

None Normal $\mathrm{x}$

Clock Other (specify)

Location within house 
$X$. Significant external loads: Irrigation pumps $\mathrm{X}$ Welder

Outside lights Other (specify)

Frequency of use 3 hrs every 3 rd day

$X I$. Exp. imental retrofit installation:

Description A/C upgrade (SEER 6 to 13.1)

Florida Heat Pump SE053-1

Actual installation cost $\$ 3125.00$ 


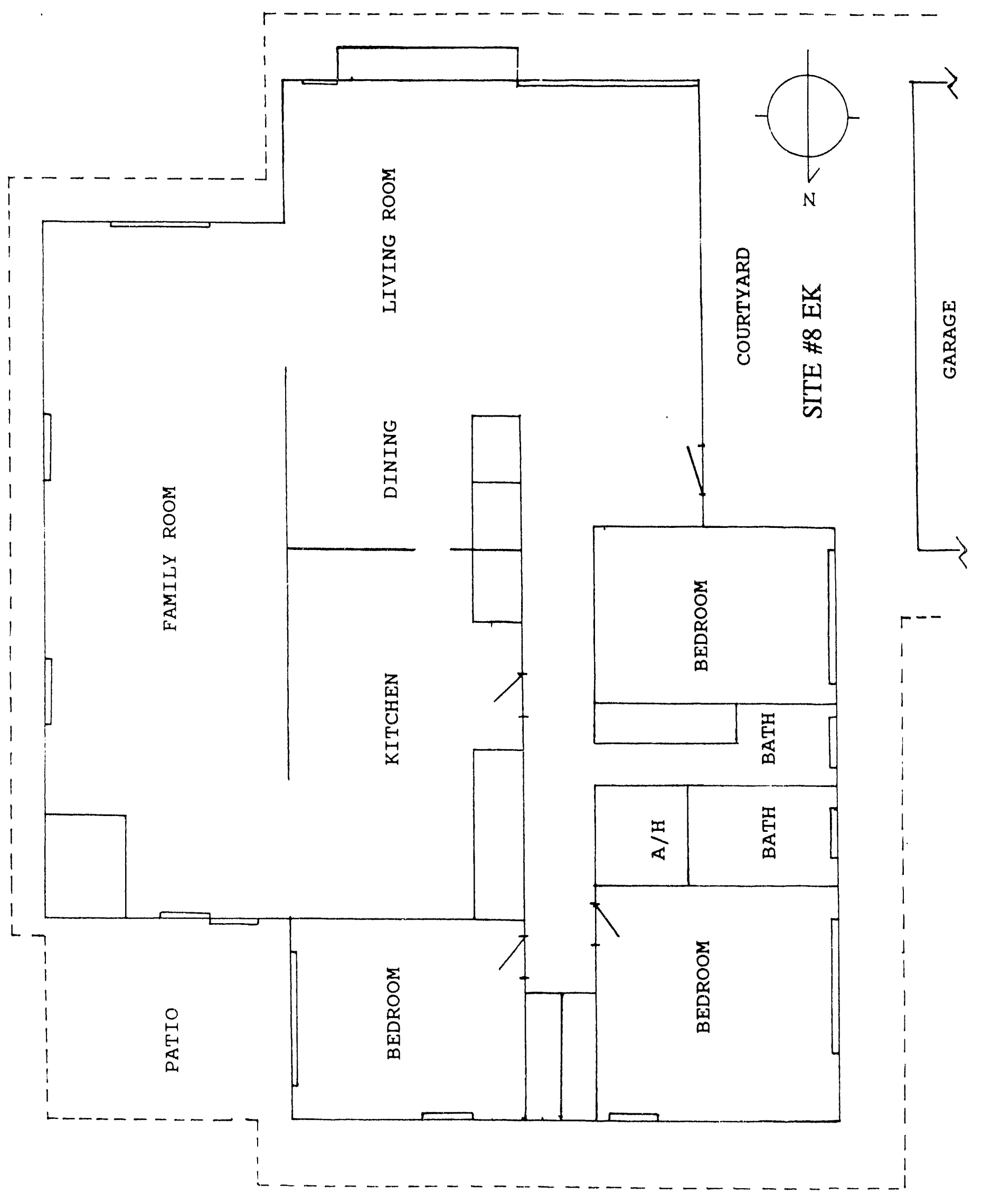



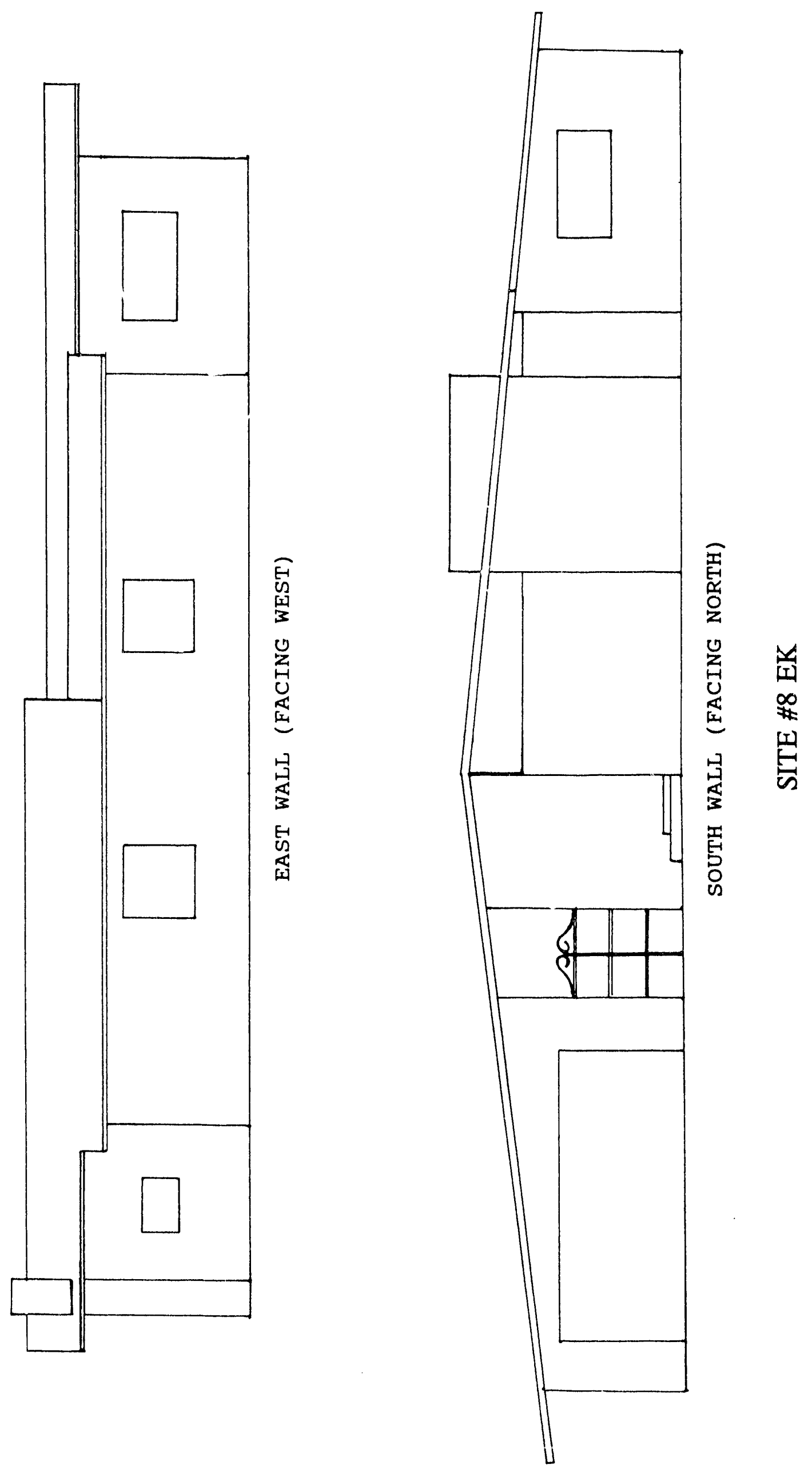

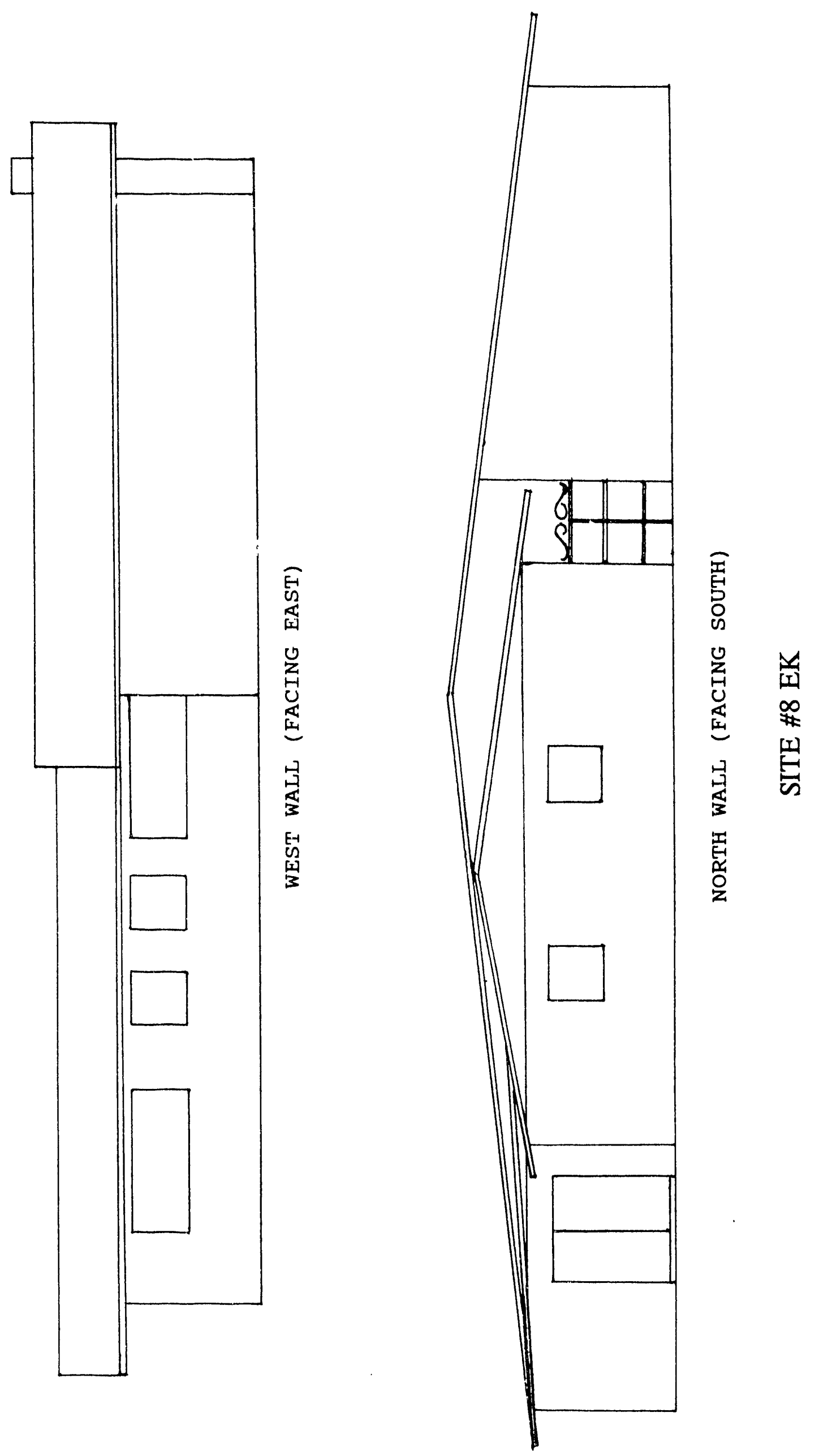
INFILTRATION RATE EVALUATION FACTORS

\begin{tabular}{|c|c|c|c|}
\hline $\begin{array}{l}\text { Building } \\
\text {-omponent }\end{array}$ & Good & Average & Poor \\
\hline $\begin{array}{l}\text { Windows } \\
\text { and } \\
\text { doors }\end{array}$ & $\begin{array}{l}\text { Window and door frames } \\
\text { caulked. Window and door } \\
\text { sashes well fitting and weather- } \\
\text { stripped or storm windows and } \\
\text { doors with good fit. }\end{array}$ & $\begin{array}{l}\text { Window and door frames } \\
\text { caulked or window and door } \\
\text { sashes weatherstripped or } \\
\text {. poorly fitting storm doors and } \\
\text { windows. }\end{array}$ & $\begin{array}{l}\text { No caulking on window and } \\
\text { door frames. No weather- } \\
\text { stripping. No storm doors or } \\
\text { windows. }\end{array}$ \\
\hline $\begin{array}{l}\text { Walls and } \\
\text { electrical } \\
\text { outlets }\end{array}$ & $\begin{array}{l}\text { Ceiling and noor joints and } \\
\text { corners well sealed. Elec- } \\
\text { trical outlets with gaskets. No } \\
\text { holes around plumbing penetra- } \\
\text { tions. }\end{array}$ & $\begin{array}{l}\text { X. Some cracks in ceiling and } \\
\text { floor joints and corners. No } \\
\text { gaskets on electrical outlets. } \\
\text { Fewer than three plumbing } \\
\text { penetrations with holes } \\
\text { around them. }\end{array}$ & $\begin{array}{l}\text { Many cracks in ceiling, } \\
\text { floor joints, and corners. No } \\
\text { gaskets on electrical outlets. } \\
\text { Three or more plumbing } \\
\text { penetrations with holes } \\
\text { around them. }\end{array}$ \\
\hline $\begin{array}{l}\text { Attic } \\
\text { noor } \\
\text { (ceiling) }\end{array}$ & $\begin{array}{l}\text { X. No cracks in attic noor. No } \\
\text { air shafts around flues. No } \\
\text { holes around ducts, pipes, or } \\
\text { wiring penetrating attic floor. } \\
\text { No t cessed light fixtures. No } \\
\text { trap door or weatherstripped } \\
\text { trap door to attic. }\end{array}$ & $\begin{array}{l}\text { Some cracks in attic floor. } \\
\text { No air shafts around flues. } \\
\text { Some holes around ducts, pipes, } \\
\text { or wiring penetrating attic floor. } \\
\text { Fewer than three recessed light } \\
\text { fixtures. Unweatherstripped } \\
\text { trap door to attic. }\end{array}$ & $\begin{array}{l}\text { Many cracks in attic floor. } \\
\text { Air shafts around nues. } \\
\text { Holes around ducts, pipes, or } \\
\text { wiring penetrating attic foor. } \\
\text { More than three recessed light } \\
\text { fixtures. Uncovered attic } \\
\text { access. }\end{array}$ \\
\hline $\begin{array}{l}\text { Heating } \\
\text { system } \\
\text { and water } \\
\text { healer }\end{array}$ & $\begin{array}{l}\text { XX Both furnace and water } \\
\text { heater electric. If fossil fuel } \\
\text { fired, both in unconditioned } \\
\text { space. }\end{array}$ & $\begin{array}{l}\text { One fossil fuel-fired unit in } \\
\text { living space with vent } \\
\text { damper. The other in uncondi- } \\
\text { tioned space. }\end{array}$ & $\begin{array}{l}\text { At least one fossil fuel-fired } \\
\text { unit in living space without } \\
\text { vent damper. }\end{array}$ \\
\hline $\begin{array}{l}\text { Fireplace } \\
\text { or wood } \\
\text { stove }\end{array}$ & $\begin{array}{l}\text { Q Sealed combustion wood } \\
\text { stove or fireplace with well- } \\
\text { fitting damper and glass doors } \\
\text { or no fireplace. }\end{array}$ & $\begin{array}{l}0 \text { Poorly sealed wood stove } \\
\text { or fireplace with either well- } \\
\text { ritting damper or glass doors. }\end{array}$ & $\begin{array}{l}\text { Both a wood stove and fire- } \\
\text { place or a fireplace with } \\
\text { poorly fitting damper and no } \\
\text { glass doors. }\end{array}$ \\
\hline $\begin{array}{l}\text { Ductwork } \\
\text { and } \\
\text { noor }\end{array}$ & $\begin{array}{l}\text { No ductwork and few noor } \\
\text { penetrations or all ductwork } \\
\text { in conditioned space and } \\
\text { no floor penetration. }\end{array}$ & $\begin{array}{l}\text { Ductwork in conditioned } \\
\text { basement and few noor } \\
\text { penetrations. }\end{array}$ & 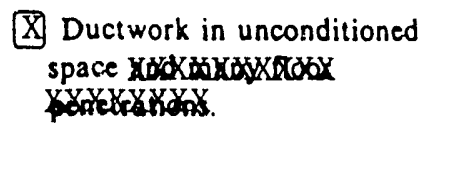 \\
\hline $\begin{array}{l}\text { Vents in } \\
\text { conditioned } \\
\text { space }\end{array}$ & $\begin{array}{l}\text { No undampered vents and } \\
\text { fewer than three dampered } \\
\text { vents. }\end{array}$ & $\begin{array}{l}\text { Fewer than three undampered } \\
\text { vents or at least three } \\
\text { dampered vents. }\end{array}$ & $\begin{array}{l}\square \text { More than three undampered } \\
\text { vents. }\end{array}$ \\
\hline Lifestyle & $\begin{array}{l}\text { Fewer than six entrances and } \\
\text { exits per day. }\end{array}$ & $\begin{array}{l}\text { Six to thirteen entrances } \\
\text { and exits per day. }\end{array}$ & $\begin{array}{l}\square \text { More than thirteen } \\
\text { entrances and exits per day. }\end{array}$ \\
\hline
\end{tabular}




\section{POST-RETROFIT AUDIT DATA FORM}

Retrofit program name Monitoring Conservative Retrofits in Single Family Bldgs Sponsoring organization(s) DOE/FPL/State of FL

Experimental site designation

EK (非)

Interviewer name CSR

Date of interview

$11 / 22 / 92$

I. General information from householder

1. Name Edward Kizak

2. Street address

City State

3. Phone number

4. Own Rent

5. If householder different from that of "Pre-Retrofit Audit," or if tenure has changed, record below:

Change in householder Date of change

Change in tenure Date of change

6. Number of occupants, by age group:

\begin{tabular}{ll}
\multicolumn{1}{c}{ Age group } & Number \\
\hline Preschool & \\
School age & \\
Adult, but not retired & $-\frac{1}{1}$ \\
Retired &
\end{tabular}

How many are generally home all day?

Weekday $\frac{1}{2}$
Weekend

II. Significant changes since pre-retrofit audit NONE

(The remaining questions refer to the time since the energy-saving improvements were made to the house).

1. Have there been any important changes or additions to the house, such as (check appropriate):

Rooms added Siding added

Garage/carport added Exterior paint 
Porch added New roof

Other (specify)

2. Other than changes of this program, has the main heating or cooling system been changed, such as (check appropriate): No

New furnace/boiler

New air conditioner

New heat pump

Modified furnace/boiler (specify)

Other (specify)

3. Are the same number of rooms still heated and cooled? (If not, specify) Yes

4. Have any space heaters been added/subtracted? (Fireplaces, wood stoves, electric, fossil fuel, etc.) Yes $\longrightarrow$ No $\mathrm{X}$

If so, specify

Has the use of these heaters changed during this last winter?

Used more this winter _ Less _ Same _ Do not know

5. Has the hot water heating system changed? Yes ___ No $\mathrm{X}$ If so, specify

6. Have any major appliances been added/subtracted? (Stoves, ovens, washers, dryers, etc.) No

If so, specify

7. Is there: An attic fan No Whole house fan No

If so, has the use of these fans changed during this last summer?

Used more this summer _ Less _ Same _ Do not know

8. Other than changes of this program, have any other energy-saving cinanges been installed this past year? Yes _ No _ X

If yes, specify:

Ceiling insulation added Weatherstripping

Wall insulation added Caulking

Storm windows added Hot water flow restrictors

Storm doors added Clock thermostat

Other (specify) 
9. Have you or any family members taken up any new hobbies during the past year that require the use of large electrical or gas equipment?

Yes $\_$No $\mathrm{X}$

If so, specify 


\section{PRE-RETROFIT AUDIT DATA FORM}

Retrofit program name Monitoring Conservative Retrofits in Sing, le Family Bldgs

Sponsoring organization(s)

DOE/FPL/State of FL

Experimental site designation $\mathrm{WH}($ 非)

Interviewer name

CSR

Date of interview

$9 / 3 / 91$

I. General information from householder

1. Name William Hendley

2. Street address 2200 Ohio Street

City Melbourne

State

FL $\quad 32904$

3. Phone number $\quad 727-7596$

4. Number of occupants, by age group:

\begin{tabular}{ll}
\multicolumn{1}{c}{ Age group } & Number \\
\hline Preschool & -1 \\
School age & -1 \\
Adult, but not retired & - \\
Retired &
\end{tabular}

How many are generally home all day?

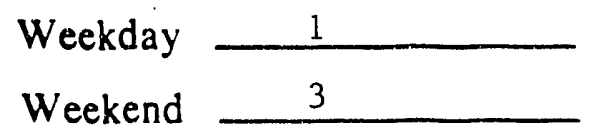

5 Approximate age of house years

6. Names of utility distributors:

Electric Florida Power \& Light

Gas

Oil

(Note: Auditor should have homeowiter sign a release authorizing release by these utilities of fuel bills for preceding two years.)

7. Oxin $\mathrm{X}$ Rent

8. Length of time at this address years

I1. General description of house

1. Type: S-F detached $\mathrm{X}$ S-F attached

Mobile home Other (specify) 
2. Style: One-story $\mathrm{x}$ Two-story Split-level

One-and-a-half-story

3. Foundation: Basement Crawl space Slab Other (specify)

4. Exterior colors: Roof Brown Walls Dark brick

5. Layout, dimensions, orientation: Use attached House Sketch Sheet.

6. Percentage of each floor that is conditioned (heated or cooled):

\begin{tabular}{lcc} 
Floor of house & $\begin{array}{c}\text { Percentage } \\
\text { heated }\end{array}$ & $\begin{array}{c}\text { Percentage } \\
\text { cooled }\end{array}$ \\
\hline $\begin{array}{l}\text { Basement } \\
\text { First floor }\end{array}$ & $100 \%$ & $100 \%$ \\
Serond floor & & \\
Third floor & & \\
Other (specify) & &
\end{tabular}

7. Infiltration: Use attached Infiltration Rate Evaluation Factors sheet.

\&. Rooms typically closed off None

III. Major appliances

1. Domestic hot water

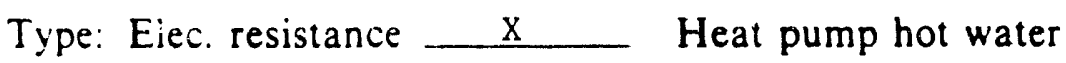

Gas Solar

Other (specify)

Nameplate infurmation, as available:

Manufacturer Reliance

Model $5-40-2 \mathrm{KR} 58 \mathrm{FJ}$

Tank capacity (gallons) 40 gal

SEER

Tank insulation ( $R$-value)

Location Garage

Hol water temperature (measured with thermometer) see data ${ }^{\circ} \mathrm{F}$

2. Solar devices

None $\mathrm{X}$ Domestic hot water Swimming pool heater Space heating Other (specify) 
3. Other major appliances, by fuel type:

\begin{tabular}{|c|c|c|c|c|}
\hline Appliance & None & Electric & Gas & $\begin{array}{l}\text { Other } \\
\text { (specify) }\end{array}$ \\
\hline Cooking range/oven & & $\mathrm{x}$ & & \\
\hline Clothes washer & & $\mathrm{x}$ & & \\
\hline Clothes dryer & & $\mathrm{x}$ & & \\
\hline Refrigerator/freezer & & $\mathrm{x}$ & & \\
\hline Separate freezer & & & & \\
\hline Conventional oven & & $\mathrm{x}$ & & \\
\hline Microwave oven & & $\mathrm{x}$ & & \\
\hline Dishwasher & & $\mathrm{x}$ & & \\
\hline Whole house fan & & & & \\
\hline Attic fan & & & & \\
\hline Water bed heater & & & & \\
\hline Other & & & & \\
\hline
\end{tabular}

IV. Ceiling area and insulation

(If different parts of the ceiling have different insulation, treat each of these sub-areas separately.)

\begin{tabular}{cccc} 
Sub-area & $\begin{array}{c}\text { Area } \\
\left(\mathrm{ft}^{2}\right)\end{array}$ & $\begin{array}{c}\text { Insulation } \\
(\mathrm{R})\end{array}$ & $\begin{array}{c}\text { Insulation } \\
\text { type }\end{array}$ \\
\hline $\begin{array}{c}1 . \\
2 .\end{array}$ & 1810 & 19 & blown \\
3. & & & \\
Total _1810 $\left(\mathrm{ft}^{2}\right)$ & &
\end{tabular}

V. Wall area and insulation

(If different parts of wall have different materials, treat each of these sub-areas separately.)

Exterior wall construction: (1)Wood; 2) Brick (3) Stone; (4) Stucco;

(5) Concrete block; (6) Metal;

(7) Other (specify)

\begin{tabular}{|c|c|c|c|}
\hline & $\begin{array}{l}\text { Area } \\
\left(\mathrm{ft}^{2}\right)\end{array}$ & $\begin{array}{c}\text { Insulation } \\
\text { (R) }\end{array}$ & $\begin{array}{l}\text { Exterior wall } \\
\text { construction }\end{array}$ \\
\hline
\end{tabular}
1. $2200 \mathrm{ft}^{2}$
2.
3.
Total $2200 .\left(\mathrm{ft}^{2}\right)$ 
V1. Floor area and insulation (bottcm floor only)

(If different parts of floor have different materials, treat each of these sub-areas separately.)

\begin{tabular}{|c|c|c|c|c|c|}
\hline Sub-area & $\begin{array}{l}\text { Area } \\
\left(\mathrm{ft}^{2}\right)\end{array}$ & $\begin{array}{l}\text { Insulation } \\
(\mathrm{R})\end{array}$ & $\begin{array}{l}\text { Percent } \\
\text { carpeted }\end{array}$ & $\begin{array}{l}\text { What is below? } \\
\text { (slab, crawl space, } \\
\text { basement?) }\end{array}$ & $\begin{array}{c}\text { Insulation } \\
\text { type }\end{array}$ \\
\hline $\begin{array}{l}1 . \\
2 . \\
3 .\end{array}$ & 1810 & - & $46 \%$ & slab & -- \\
\hline
\end{tabular}

V11. W'indows and glass doors

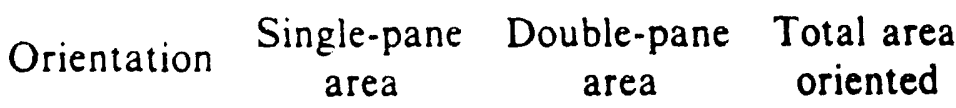

Front of $\quad 76 \mathrm{ft}^{2} \quad 76 \mathrm{ft}^{2}$

house

Left side

of house

$13.5 \mathrm{ft}^{2}$

$13.5 \mathrm{ft}^{2}$

Right side

$9 \mathrm{ft}^{2}$

$9 \mathrm{ft}^{2}$

of house

Back of

$84 \mathrm{ft}^{2}$

$84 \mathrm{ft}^{2}$

house

VIII. Doors (non-glass)

\begin{tabular}{lccc}
\multicolumn{1}{c}{ Orientation } & $\begin{array}{c}\text { Regular } \\
\text { door } \\
\text { area }\end{array}$ & $\begin{array}{c}\text { Regular door with } \\
\text { storm door or } \\
\text { thermally insulated } \\
\text { door area }\end{array}$ & $\begin{array}{c}\text { Total } \\
\text { area }\end{array}$ \\
\hline $\begin{array}{l}\text { Front of house } \\
\text { Left side of house } \\
\text { Right side of house } \\
\text { Back of house }\end{array}$ & 19.5 & & 19.5 \\
$\quad$ Totals & 36.0 & 36.0 \\
\hline
\end{tabular}


IX. Heating and cooling systems

1. Main heating system type:

Gas furnace Gas boiler (steam)

Gas boiler (hot water) Oil furnace

Oil boiler (steam) Oil boiler (hot water)

Elec. furnace Elec. ceiling Elec. baseboard

Central heat pump Window heat pump

Other (specify) Resistamce coils in $\mathrm{A} / \mathrm{H}$

If oil fired, has a flame retention head burner been installed?

Yes No

If oil boiler, is it wet base or dry base

Approximate age of system 15 years

Location of unit within house Garage Air Handler

Is this a converted coal unit? Yes No

Heating value of fuel: Gas

$$
\text { Oil }
$$

Nameplate information, as available:

Manufacturer Whirlpool

Model WCTA BBAE3

Input capacity Btu/hr

Output capacity Btu/hr

SEER

Is the pilot light turned off in the spring? Yes No N/A Energy efficiency devices present:

Vent damper IID Other (specify)

2. Distribution system

No ductwork or piping Hydronic (gravity) Hydronic (pumped) Forced-air $\mathrm{X}$

Baseboard Gravity system (no fans)

Other (specify) 
If ductwork or piping present:

Total length of ducts or piping 132 ft supply $+64^{\prime}$ return

Percentage of ducts or piping in unconditioned (non-living) spaces $100 \%$ Are ducts or piping in unconditioned spaces insulated?

Yes $\_$No If yes, approximate R-value $\quad 5$

3. Main cooling system type:

None

Window $A / C$

Central A/C

$\mathrm{X}$

Evaporative cooler Central heat pump

Window heat pump Other (specify)

Approximate age of system 15 years

Nameplate information, as available

Manufacturer Whirlpool

Model 36 TE $703 \quad 6605$

Input capacity Btu/hr

Output capacity 36,000 But/hr

SEER approximately 8.5

Location(s) within house West wall of Garage

4. Secondary heating systems:

No. fireplaces 1

No. fireplace inserts

No. wood stuves

No. electric space heaters

No. fossil fuel space heaters

Other (specify)

How often are these used?

Not used

5. Thermostat:

None Normal $\mathrm{X}$

Clock Other (specify)

Location within house 
$X$. Significant external loads:

Irrigation pumps None

Welder

Outside lights

Other (specify)

Frequency of use

XI. Experimental retrofit installation:

Description A/C upgrade

Actual installation cost $\$ \quad 2800.00$ 


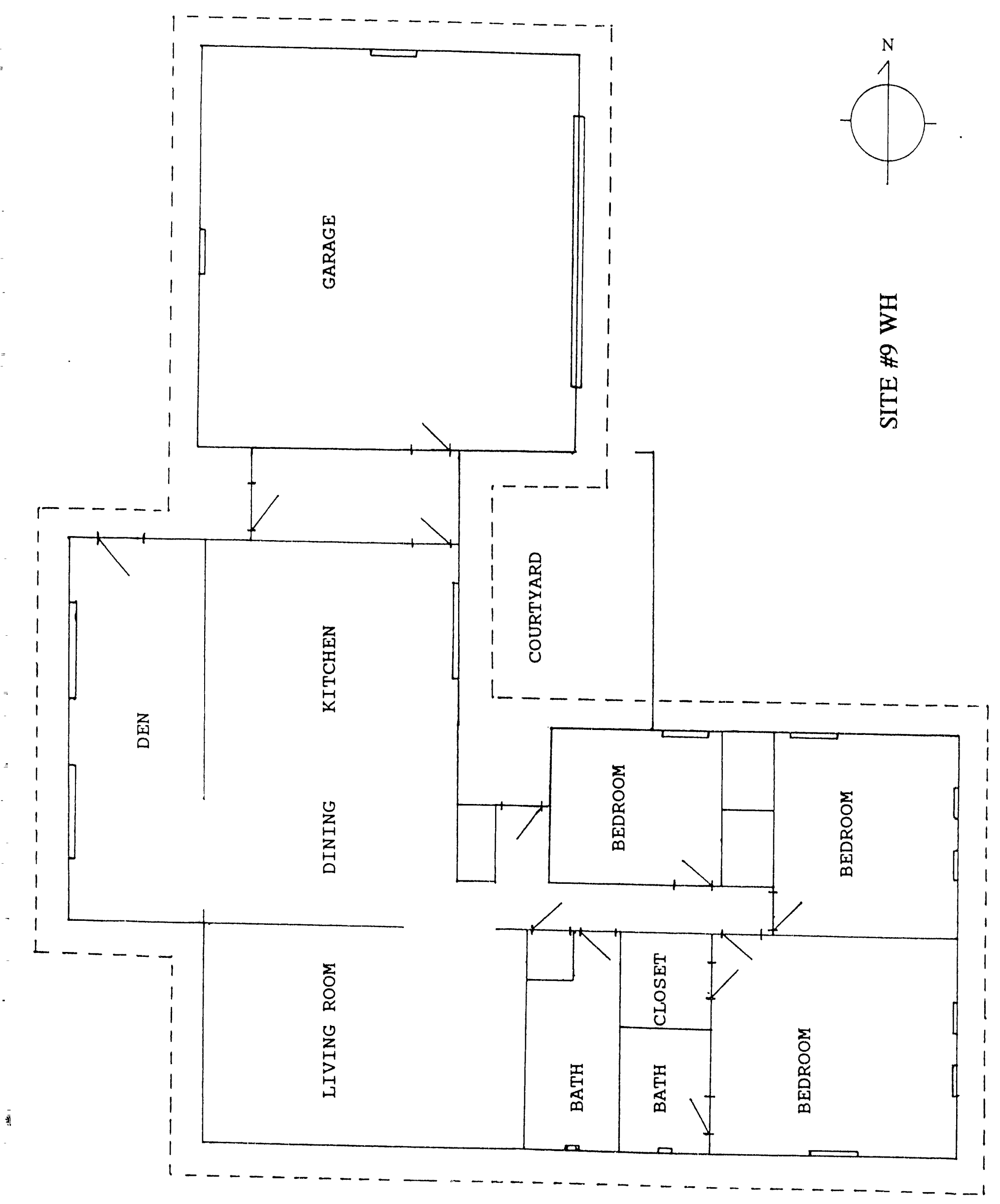



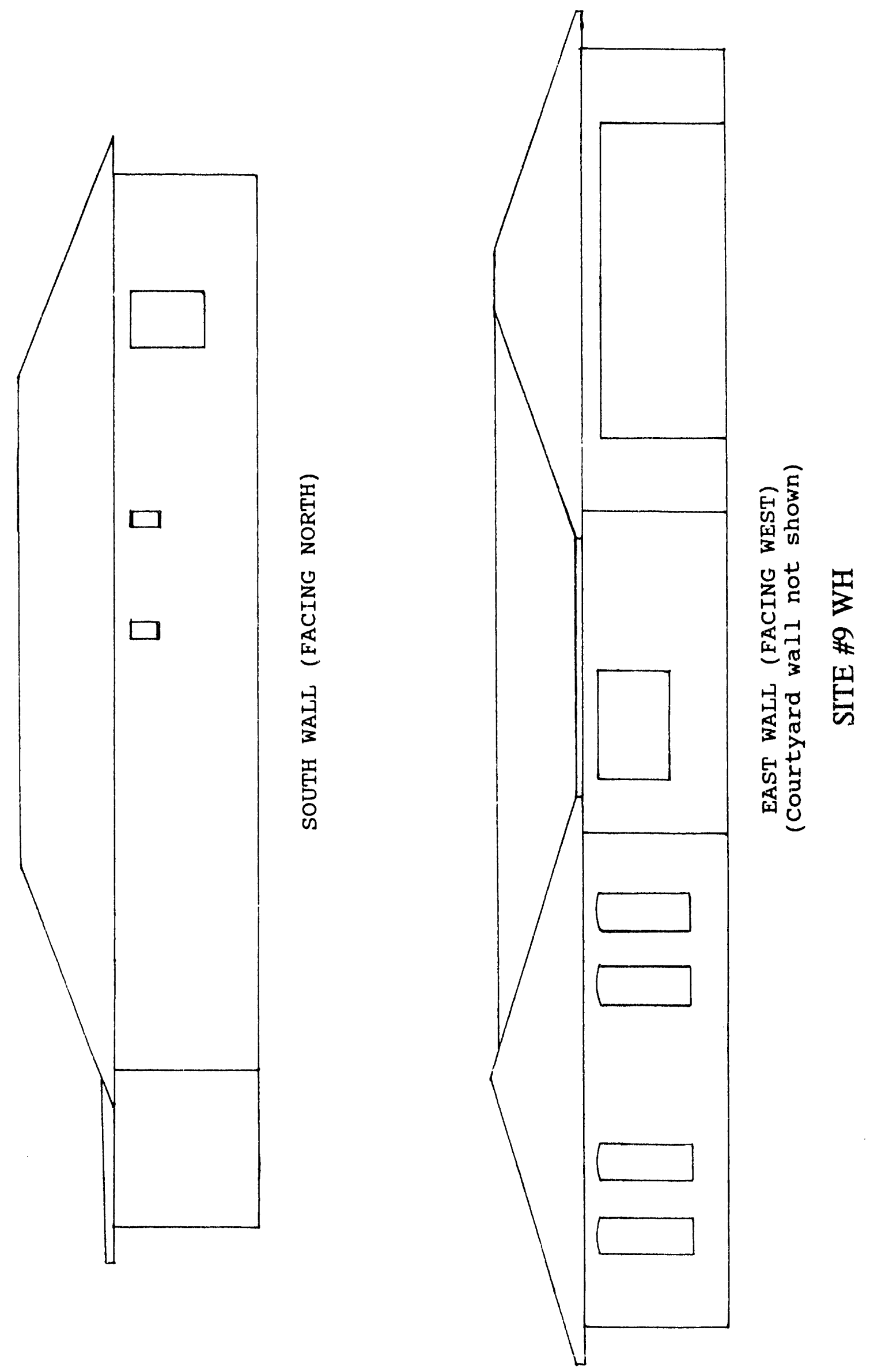

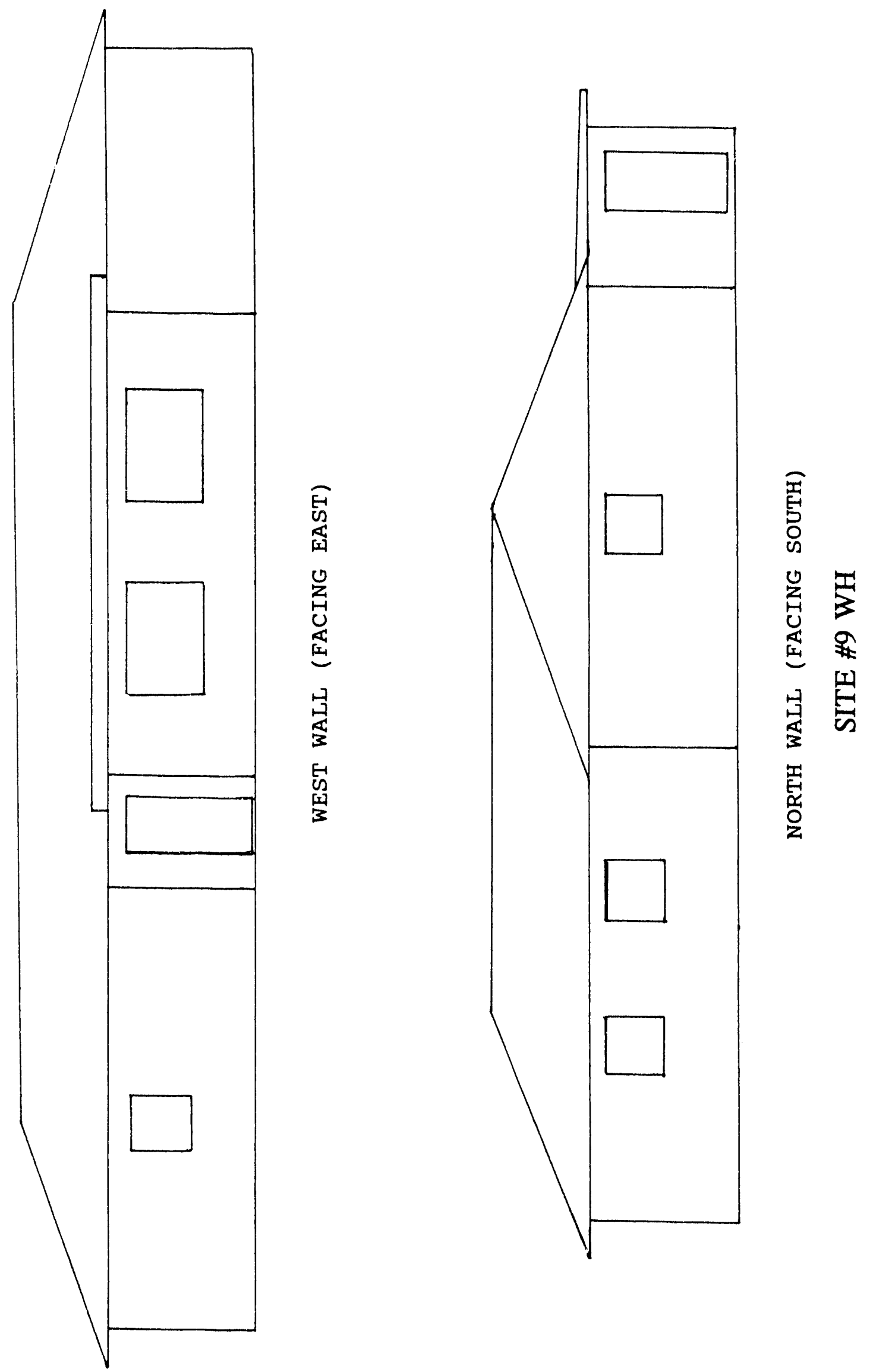
INFILTRATION RATE EVALUATION FACTORS

\begin{tabular}{|c|c|c|c|}
\hline $\begin{array}{l}\text { Building } \\
\text { component }\end{array}$ & Good & Average & Poor \\
\hline $\begin{array}{l}\text { Winoous } \\
\text { and } \\
\text { doors }\end{array}$ & $\begin{array}{l}\text { Window and door frames } \\
\text { caulked. Window and door } \\
\text { sashes well fitting and weather- } \\
\text { stripped or storm windows and } \\
\text { doors with good fit. }\end{array}$ & $\begin{array}{l}\text { Window and door frames } \\
\text { caulked or window and door } \\
\text { sashes weatherstripped or } \\
\text { poorly fitting storm doors and } \\
\text { windows. }\end{array}$ & $\begin{array}{l}\text { No caulking on window and } \\
\text { door frames. No weather- } \\
\text { stripping. No storm doors or } \\
\text { windows. }\end{array}$ \\
\hline $\begin{array}{l}\text { Walls and } \\
\text { electrical } \\
\text { outlets }\end{array}$ & 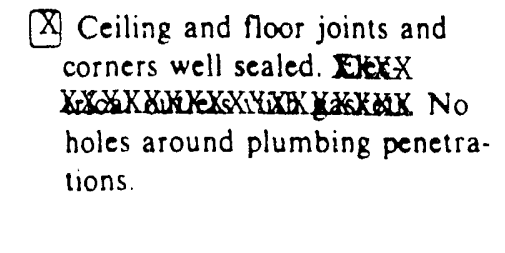 & $\begin{array}{l}\text { Some cracks in ceiling and } \\
\text { floor joints and corners. No } \\
\text { gaskets on electrical outlets. } \\
\text { Fewer than three plumbing } \\
\text { penetrations with holes } \\
\text { around them. }\end{array}$ & $\begin{array}{l}\text { Many cracks in ceiling, } \\
\text { foor joints, and corners. No } \\
\text { gaskets on electrical outlets. } \\
\text { Three or more plumbing } \\
\text { penetrations with holes } \\
\text { around them. }\end{array}$ \\
\hline $\begin{array}{l}\text { Attic } \\
\text { noor } \\
\text { (ceiling) }\end{array}$ & $\begin{array}{l}\text { X No cracks in attic floor. No } \\
\text { air shafts around flues. No } \\
\text { holes around ducts, pipes, or } \\
\text { wiring penetrating attic noor } \\
\text { No recessed light fixtures. No } \\
\text { trap door or weatherstripped } \\
\text { trap donr to attic. }\end{array}$ & $\begin{array}{l}\text { Some cracks in attic floor. } \\
\text { No air shafts around flues. } \\
\text { Some holes around ducts, pipes, } \\
\text { or wiring penetrating attic foor. } \\
\text { Fewer than three recessed light } \\
\text { fixtures. Unweatherstripped } \\
\text { trap door to attic. }\end{array}$ & $\begin{array}{l}\text { Many cracks in attic floor. } \\
\text { Air shafts around flues. } \\
\text { Holes around ducts, pipes, or } \\
\text { wiring penetrating attic floor. } \\
\text { More than three recessed light } \\
\text { fixtures. Uncovered attic } \\
\text { access. }\end{array}$ \\
\hline $\begin{array}{l}\text { Heating } \\
\text { system } \\
\text { and water } \\
\text { heater }\end{array}$ & $\begin{array}{l}\text { X Both furnace and water } \\
\text { heater electric. If fossil fuel } \\
\text { fired. both in unconditioned } \\
\text { space. }\end{array}$ & $\begin{array}{l}\text { One fossil fuel-fired unit in } \\
\text { living space with vent } \\
\text { damper. The other in uncondi- } \\
\text { tioned space. }\end{array}$ & $\begin{array}{l}\text { At least one fossil fuel-fired } \\
\text { unit in living space without } \\
\text { vent damper. }\end{array}$ \\
\hline $\begin{array}{l}\text { Fireplace } \\
\text { or wixte } \\
\text { stove }\end{array}$ & $\begin{array}{l}\text { EX Sealed combustion wood } \\
\text { stove or fireplace with well- } \\
\text { fitting damper and glass doors } \\
\text { or no fireplace. }\end{array}$ & $\begin{array}{l}\square \text { Poorly sealed wood stove } \\
\text { or fireplace with either well- } \\
\text { fitting damper or glass doors. }\end{array}$ & $\begin{array}{l}\text { Both a wood stove and fire- } \\
\text { place or a fireplace with } \\
\text { poorly fitting damper and no } \\
\text { glass doors. }\end{array}$ \\
\hline $\begin{array}{l}\text { Ducitiorh } \\
\text { and } \\
\text { noor }\end{array}$ & $\begin{array}{l}\text { No ductwork and feu foor } \\
\text { penetrations or all ductwork } \\
\text { in conditioned space and } \\
\text { no noor penetration. }\end{array}$ & $\begin{array}{l}\text { Ductwork in conditioned } \\
\text { basement and few floor } \\
\text { penetrations. }\end{array}$ & 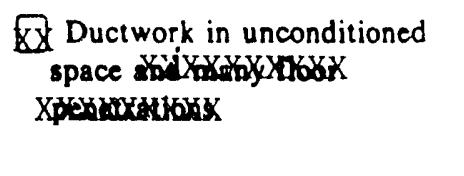 \\
\hline $\begin{array}{l}\text { Vents in } \\
\text { conditioned } \\
\text { space }\end{array}$ & $\begin{array}{l}\text { No undampered vents and } \\
\text { fewer than three dampered } \\
\text { vents }\end{array}$ & $\begin{array}{l}\mathrm{X} \text { Fewer than three undampered } \\
\text { vents or at least three } \\
\text { dampered vents. }\end{array}$ & $\begin{array}{l}\square \text { More than three undampered } \\
\text { vents. }\end{array}$ \\
\hline Lifestyle & $\begin{array}{l}\text { Tewer than six entrances and } \\
\text { exits per day. }\end{array}$ & $\begin{array}{l}\text { [X] Six to thirteen entrances } \\
\text { and exits per day. }\end{array}$ & $\begin{array}{l}\text { More than thirteen } \\
\text { entrances and exits per day. }\end{array}$ \\
\hline
\end{tabular}




\section{POST-RETROFIT AUDIT DATA FORM}

Retrofit program name Monitoring Conservative Retrofits in Single Family Bldgs Sponsoring organization(s) DOE/FPL/State of FL

Experimental site designation $\mathrm{WH}$ (非9)

Interviewer name r.SR

Date of interview

1. General information from householder

1. Name William Hendley

2. Street address

City State

3. Phone number

4. Own Rent

5. If householder different from that of "Pre-Retrofit Audit," or if tenure has changed, record below:

Change in householder Date of change

Change in tenure Date of change

6. Number of occupants, by age group:

\begin{tabular}{ll}
\multicolumn{1}{c}{ Age group } & Number \\
\hline Preschool & - \\
School age & -1 \\
Adult, but not retired & 2 \\
Retired &
\end{tabular}

How many are generally home all day?

Weekday $\frac{1}{3}$
Weekend

II. Significant changes since pre-retrofit audit None

(The remaining questions refer to the time since the energy-saving improvements were made to the house).

1. Have there been any important changes or additions to the house, such as (check appropriate):

Rooms added Siding added

Garage/carport added Exterior paint 
Porch added New roof

Other (specify)

2. Other than changes of this program, has the main heating or cooling system been changed, such as (check appropriate): No

New furnace/boiler

New air conditioner

New heat pump

Modified furnace/boiler (specify)

Other (specify)

3. Are the same number of rooms still heated and cooled? (If not, specify) Yes

4. Have any space heaters been added/subtracted? (Fireplaces, wood stoves, electric, fossil fuel, etc.) Yes _ No $\mathrm{X}$

If so, specify

Has the use of these heaters changed during this last winter?

Used more this winter _ Less _ Same $\mathrm{X}$ Do not know

5. Has the hot water heating system changed? Yes _ No $\underline{X}$ If so, specify

6. Have any major appliances been added/subtracted? (Stoves, ovens, washers, dryers, etc.) No

If so, specify

7. Is there: An attic fan No Whole house fan No

If so, has the use of these fans changed during this last summer?

Used more this summer _ Less _ Same _ Do not know

8. Other than changes of this program, have any other energy-saving cinanges been installed this past year? Yes __ No _

If yes, specify:

Ceiling insulation added

Wall insulation added

Weatherstripping

Storm windows added Caulking

Storm doors added Hot water flow restrictors

Other (specify) 
9. Have you or any family members taken up any new hobbies during the past year that require the use of large electrical or gas equipment?

Yes _ No $\mathrm{X}$.

If so, specify 


\section{PRE-RETROFIT AUDIT DATA FORM}

Retrofit program name Monitoring Conservative Retrofits in Single Family Bldgs Sponsoring organization(s) DOE/FPL/State of FL

Experimental site designation

Interviewer name
CSR
JP (非10)

Date of interview $9 / 7 / 91$

I. General information from householder

1. Name Jon Peterson

2. Street address 3081 Grace Street

City Melbourne State FL 32904

3. Phone number $951-7038$

4. Number of occupants, by age group:

\begin{tabular}{ll}
\multicolumn{1}{c}{ Age group } & Number \\
\hline Preschool & \\
School age & 1 \\
Adult, but not retired & -2 \\
Retired &
\end{tabular}

How many are generally home all day?

Weekday $\frac{1}{3}$
Week nd

5. Approximate age of house 25 years

6. Names of utility distributors:

Electric Florida Power \& Light

Gas

Oil

(Note: Auditor should have homeowner sign a release authorizing release by these utilities of fuel bills for preceding two years.)

7. Own $\mathrm{X}$ Rent

8. Length of time at this address 5

II. General description of house

1. Type: S-F detached $\mathrm{x}$ S-F attached

Mobile home Other (specify) 
2. Style: One-story $\mathrm{x}$ Two-story Three-story Split-level One-and-a-half-story

3. Foundation: Basement Crawl space Slab

Other (specify)

4. Exterior colors: Roof Brown Walls Tan

5. Layout, dimensions, orientation: Use attached House Sketch Sheet.

6. Percentage of each floor that is conditioned (heated or cooled):

\begin{tabular}{lccc} 
Floor of house & $\begin{array}{c}\text { Percentage } \\
\text { heated }\end{array}$ & $\begin{array}{c}\text { Percentage } \\
\text { cooled }\end{array}$ & NA \\
\hline $\begin{array}{l}\text { Basement } \\
\text { First floor }\end{array}$ & $81 \%$ & $81 \%$ \\
$\begin{array}{l}\text { Second floor } \\
\text { Third floor }\end{array}$ & & \\
Other (specify) & &
\end{tabular}

7. Infiltration: Use attached Infiltration Rate Evaluation Factors sheet.

8. Rooms typically closed off Florida room, utility room

III. Major appliances

1. Domestic hot water

Type: Elec. resistance $\mathrm{X} \quad$ Heat pump hot water

Gas Solar

Other (specify)

Nameplate information, as available:

Manufacturer Rheem

Model 666H-40D EOC

Tank capacity (gallons) $\quad 40$ gal

SEER --

Tank insulation ( $R$-value)

Location Utility room

Hot water temperature (measured with thermometer) see data ${ }^{\circ} \mathrm{F}$

2. Solar devices

None $\mathrm{x}$ Domestic hot water Swimming pool heater

Space heating Other (specify) 
3. Other major appliances, by fuel type:

\begin{tabular}{|c|c|c|c|c|}
\hline Appliance & None & Electric & Gas & $\begin{array}{l}\text { Other } \\
\text { (specify) }\end{array}$ \\
\hline Cooking range/oven & & $\mathrm{x}$ & & \\
\hline Clothes washer & & $\mathrm{x}$ & & \\
\hline Clothes dryer & & & & \\
\hline Refrigerator/freezer & & $\mathrm{x}$ & & \\
\hline Separate freezer & & $\mathrm{x}$ & & \\
\hline Conventional oven & & & & \\
\hline Microwave oven & & $\mathrm{x}$ & & \\
\hline Dishwasher & & & & \\
\hline Whole house fan & & & & \\
\hline Attic fan & & & & \\
\hline Water bed heater & & & & \\
\hline Other & & & & \\
\hline
\end{tabular}

IV. Ceiling area and insulation

(If different parts of the ceiling have different insulation, treat each of these sub-areas separately.)

\begin{tabular}{|c|c|c|c|}
\hline Sub-area & $\begin{array}{l}\text { Area } \\
\left(\mathrm{ft}^{2}\right)\end{array}$ & $\begin{array}{l}\text { Insulation } \\
(\mathrm{R})\end{array}$ & $\begin{array}{l}\text { Insulation } \\
\text { type }\end{array}$ \\
\hline 1. & 1352 & $<7$ & batts \\
\hline $\begin{array}{l}2 . \\
3 .\end{array}$ & 320 & None & \\
\hline Total & \multicolumn{3}{|c|}{$1672 \mathrm{ft}^{2}$} \\
\hline
\end{tabular}

V. Wall area and insulation

(If different parts of wall have different materials, treat each of these sub-areas separately.)

Exterior wall construction: (1)Wood; (2) Brick; (3) Stone; (4) Stucco;

(5) Concrete block: (6) Metal;

(7) Other (specify)

\begin{tabular}{|c|c|c|c|}
\hline & $\begin{array}{l}\text { Area } \\
\left(\mathrm{ft}^{2}\right)\end{array}$ & $\begin{array}{l}\text { Insulation } \\
\text { (R) }\end{array}$ & $\begin{array}{l}\text { Exterior wall } \\
\text { construction }\end{array}$ \\
\hline
\end{tabular}

1. $2040 \mathrm{ft}^{2}$ unknown

2.

3.

Total $2040\left(\mathrm{ft}^{2}\right)$ 
VI. Floor area and insulation (bottom floor only) (If different parts of floor have different materials, treat each of these sub-areas separately.)

\begin{tabular}{cccccc} 
Sub-area & $\begin{array}{c}\text { Area } \\
\left(\mathrm{ft}^{2}\right)\end{array}$ & $\begin{array}{c}\text { Insulation } \\
(\mathrm{R})\end{array}$ & $\begin{array}{c}\text { Percent } \\
\text { carpeted }\end{array}$ & $\begin{array}{c}\text { What is below? } \\
\text { (slab, crawl space, } \\
\text { basement?) }\end{array}$ & $\begin{array}{c}\text { Insulation } \\
\text { type }\end{array}$ \\
\hline $\begin{array}{c}1 . \\
2 .\end{array}$ & 1672 & - & $27 \%$ & slab & -- \\
3. \\
Total 1.572
\end{tabular}

VII. Windows and glass doors

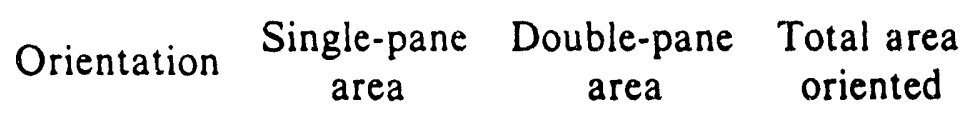

Front of $\quad 48 \mathrm{ft}^{2} \quad 48 \mathrm{ft}^{2}$

house

Left side $\quad 30 \mathrm{ft}^{2}$

of house

$30 \mathrm{ft}^{2}$

Right side

$29 \mathrm{ft}^{2}$

of house

$48 \mathrm{ft}^{2}$

(1) house

Back of house

$69 \mathrm{ft}^{2}$

$69 \mathrm{ft}^{2}$

$29 \mathrm{ft}^{2}$

house

VIII. Doors (non-glass)

\begin{tabular}{llcc} 
Orientation & $\begin{array}{c}\text { Regular } \\
\text { door } \\
\text { area }\end{array}$ & $\begin{array}{c}\text { Regular door with } \\
\text { storm door or } \\
\text { thermally insulated } \\
\text { door area }\end{array}$ & $\begin{array}{c}\text { Total } \\
\text { area }\end{array}$ \\
\hline $\begin{array}{l}\text { Front of house } \\
\text { Left side of house }\end{array}$ & 39.75 & & 39.75 \\
$\begin{array}{l}\text { Right side of houisc } \\
\text { Back of house } \\
\text { Totals }\end{array}$ & 39.75 & & \\
\hline
\end{tabular}


IX. Heating and cooling systems

1. Main heating system type:

Gas furnace Gas boiler (steam)

Gas boiler (hot water) Oil furnace $\mathrm{X}$

Oil boiler (steam) Oil boiler (hot water)

Elec. furnace Elec. ceiling Elec. baseboard

Central heat pump Window heat pump

Other (specify)

If oil fired, has a flame retention head burner been installed?

Yes No

If oil boiler, is it wet base or dry base X Approximate age of system 15 years

Location of unit within house Air handler in closet

Is this a converted coal unit? Yes $\mathrm{X}$

Heating value of fuel: Gas

Oil -

Nameplate information, as available:

Manufacturer

Model

Input capacity Btu/hr

Output capacity Btu/hr

SEER

Is the pilot light turned off in the spring? Yes $\underline{X}$ No N/A

Energy efficiency devices present:

Vent damper IID Other (specify)

2. Distribution system

No ductwork or piping Hydronic (gravity)

Hydronic (pumped) Forced-air

Baseboard Gravity system (no fans)

Other (specify) 
If ductwork or piping present:

Total length of ducts or piping 80 $\mathrm{ft}$

Percentage of ducts or piping in unconditioned (non-living) spaces $100 \%$

Are ducts or piping in unconditioned spaces insulated?

Yes $\frac{\mathrm{X}}{\longrightarrow}$ No If yes, approximate $\mathrm{R}$-value 5

3. Main cooling system type:

None Window $\mathrm{A} / \mathrm{C}$ Central A/C $\mathrm{X}$

Evaporative cooler Central heat pump

Window heat pump Other (specify)

Approximate age of system years

Nameplate information, as available

Manufacturer Snyder General Corp.

Model ACO 36GA

Input capacity $\mathrm{Btu} / \mathrm{hr}$

Output capacity Btu/hr

SEER approximately 10

Location(s) within house

4. Secondary heating systems:

No. fireplaces 1

No. fireplace inserts

No. wood stoves

No. electric space heaters

No. fossil fuel space heaters

Other (specify)

How often are these used? 6/year

5. Thermostat:

None Normal

$\mathrm{X}$

Clock Other (specify)

Location within house 
$X$. Significant external loads:

Irrigation purnps

Outside lights

Welder

Frequency of use Other (specify) Poم_ Pump see data

XI. Experimental retrofit installation:

Description Insulation upgrade $\left(<_{R 7}\right.$ to $\left.R 19\right)$

Actual installation cost $\$$

351.90 


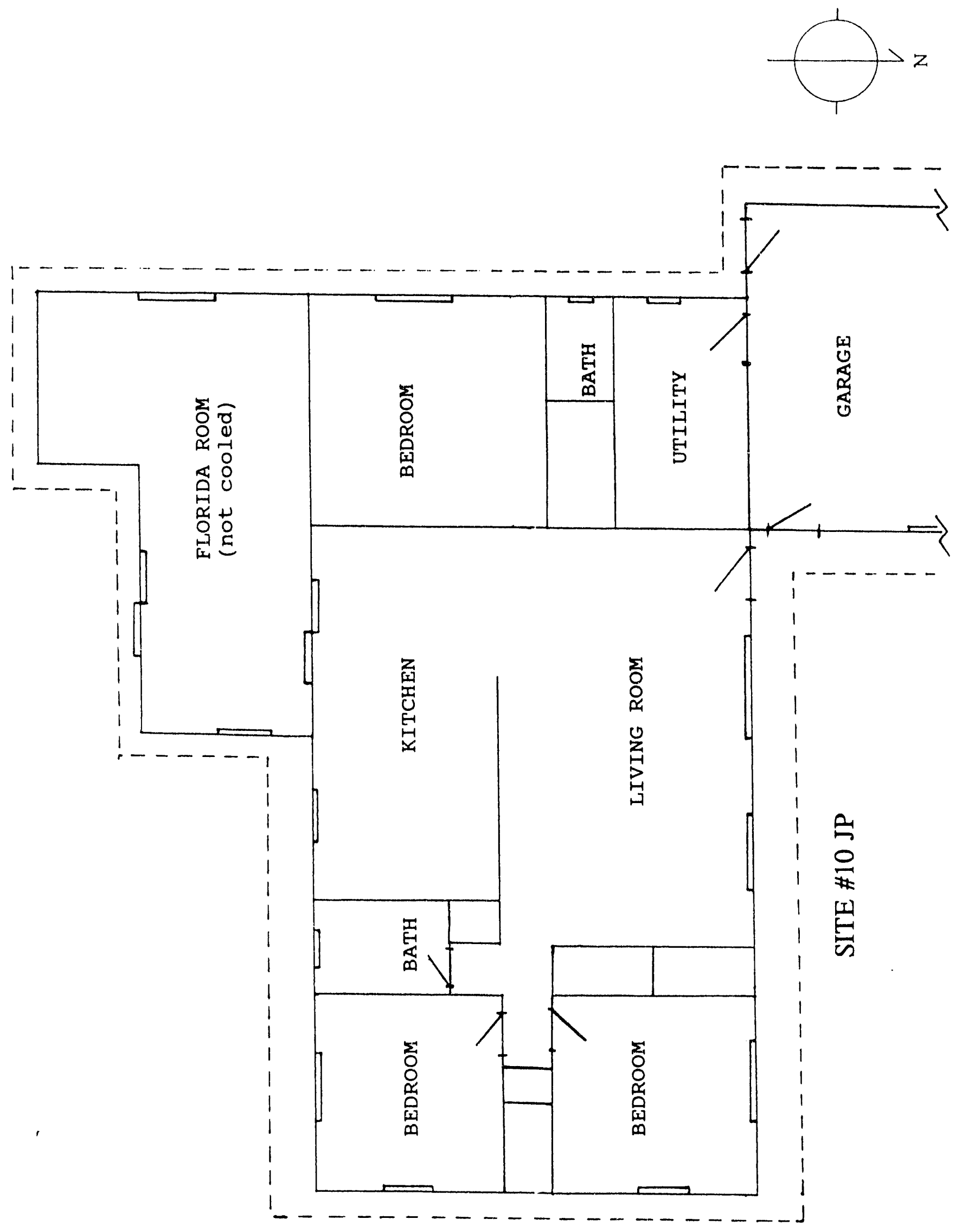



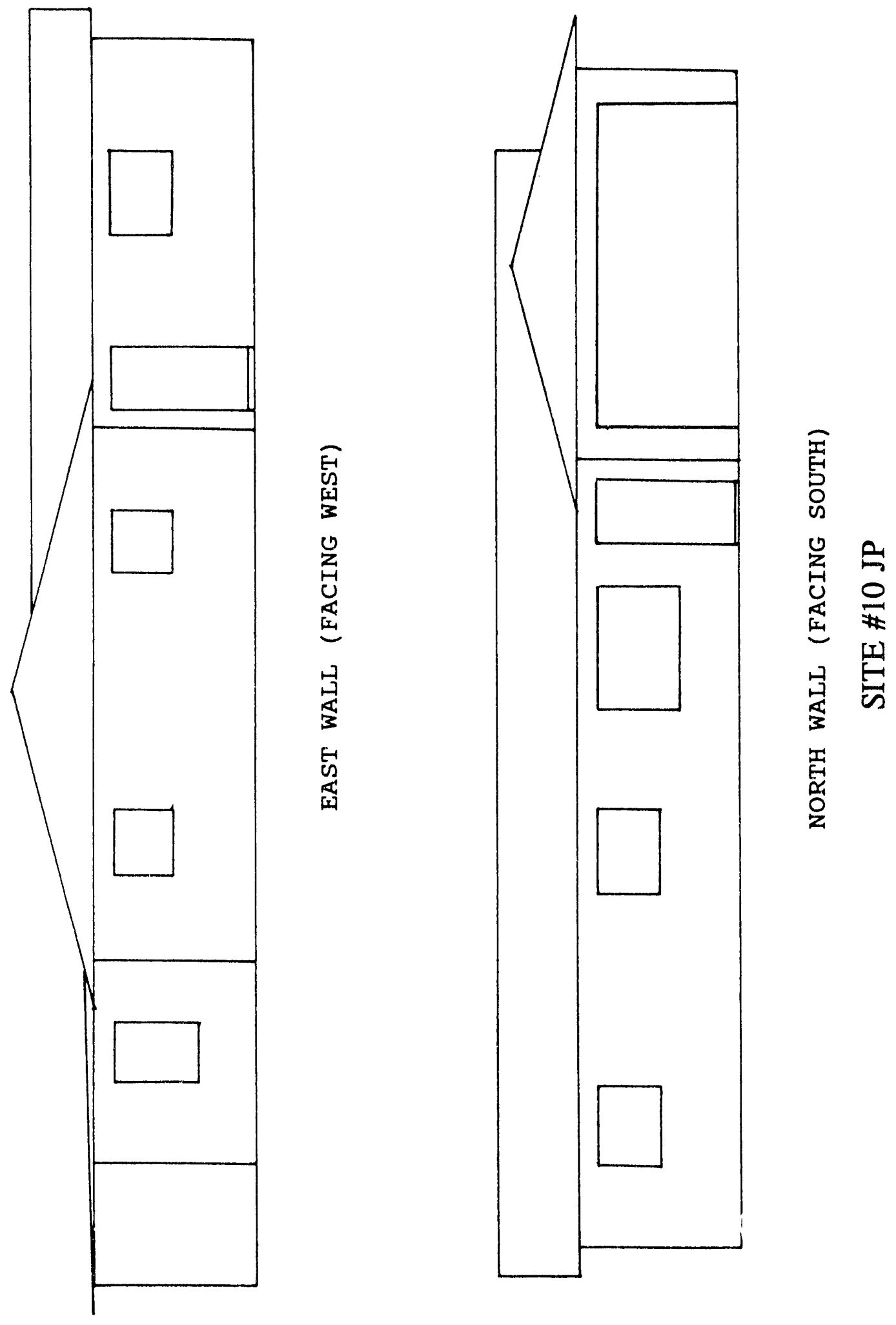

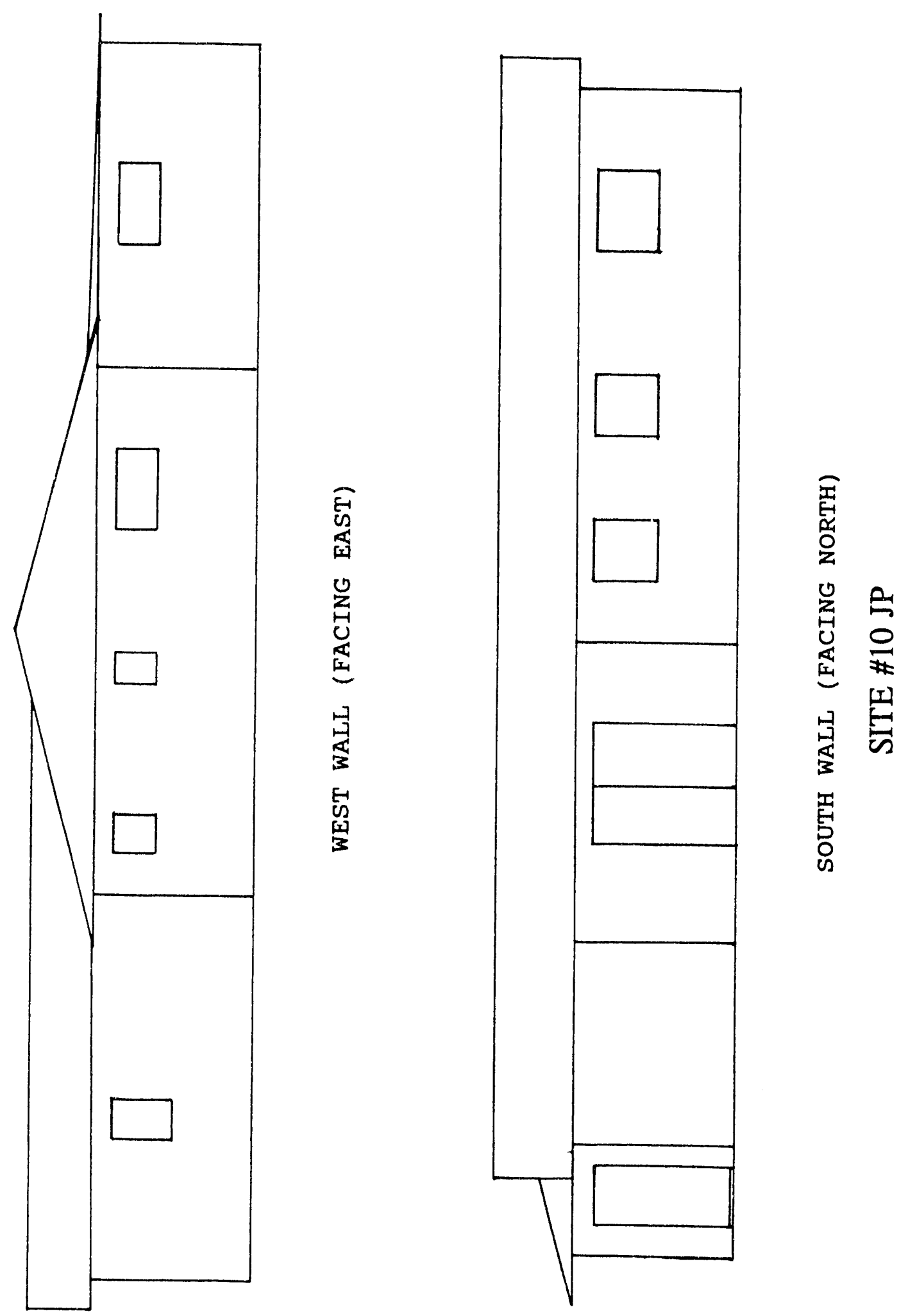
INFILTRATION RATE EVALUATION FACTORS

\begin{tabular}{|c|c|c|c|}
\hline $\begin{array}{l}\text { Building } \\
\text { component }\end{array}$ & Good & Average & Poor \\
\hline $\begin{array}{l}\text { Windows } \\
\text { and } \\
\text { doors }\end{array}$ & $\begin{array}{l}\text { Window and door frames } \\
\text { caulked. Window and door } \\
\text { sashes well fitting and weather- } \\
\text { stripped or storm windows and } \\
\text { doors with good fit. }\end{array}$ & $\begin{array}{l}\text { Window and door frames } \\
\text { caulked or window and door } \\
\text { sashes weatherstripped or } \\
\text { poorly fitting storm doors and } \\
\text { windows. }\end{array}$ & $\begin{array}{l}\square \text { No caulking on window and } \\
\text { door frames. No weather- } \\
\text { stripping. No storm doors or } \\
\text { windows. }\end{array}$ \\
\hline $\begin{array}{l}\text { Walls and } \\
\text { electrical } \\
\text { outlets }\end{array}$ & $\begin{array}{l}D \text { Ceiling and noor joints and } \\
\text { corners well sealed. Elec- } \\
\text { trical outlets with gaskets. No } \\
\text { holes around plumbing penetra- } \\
\text { tions. }\end{array}$ & $\begin{array}{l}\text { X Some cracks in ceiling and } \\
\text { floor joints and corners. No } \\
\text { gaskets on electrical outlets. } \\
\text { Fewer than three plumbing } \\
\text { penetrations with holes } \\
\text { around them. }\end{array}$ & $\begin{array}{l}\text { Many cracks in ceiling, } \\
\text { floor joints, and corners. No } \\
\text { gaskets on electrical outlets. } \\
\text { Three or more plumbing } \\
\text { penetrations with holes } \\
\text { around them. }\end{array}$ \\
\hline $\begin{array}{l}\text { Attic } \\
\text { Moor } \\
\text { (ceiling) }\end{array}$ & $\begin{array}{l}\text { No cracks in attic floor. No } \\
\text { air shafts around flues. No } \\
\text { holes around ducts, pipes, or } \\
\text { wiring penetrating attic floor. } \\
\text { No recessed light fixtures. No } \\
\text { trap door or weatherstripped } \\
\text { trap door to attic. }\end{array}$ & $\begin{array}{l}\text { Some cracks in attic floor. } \\
\text { No air shafts around nues. } \\
\text { Some holes around ducts, pipes, } \\
\text { or wiring penetrating attic noor. } \\
\text { Fewer than three recessed light } \\
\text { fixtures. Unweatherstripped } \\
\text { trap door to attic. }\end{array}$ & $\begin{array}{l}\text { Many cracks in attic floor. } \\
\text { Air shafts around flues. } \\
\text { Holes around ducts, pipes, or } \\
\text { wiring penetrating attic floor. } \\
\text { More than three recessed light } \\
\text { fixtures. Uncovered attic } \\
\text { access. }\end{array}$ \\
\hline $\begin{array}{l}\text { Heating } \\
\text { system } \\
\text { and water } \\
\text { heater }\end{array}$ & $\begin{array}{l}\text { Both furnace and water } \\
\text { heater electric. If fossil fuel } \\
\text { fired, both in unconditioned } \\
\text { space. }\end{array}$ & $\begin{array}{l}\text { One fossil fuel-fired unit in } \\
\text { living space with vent } \\
\text { damper. The other in uncondi- } \\
\text { tioned space. }\end{array}$ & $\begin{array}{l}X \text { At least one fossil fuel-fired } \\
\text { unit in living space without } \\
\text { vent damper. }\end{array}$ \\
\hline $\begin{array}{l}\text { Fireplace } \\
\text { or wood } \\
\text { stove }\end{array}$ & $\begin{array}{l}\text { Sealed combustion wood } \\
\text { stove or fireplace with well- } \\
\text { fitting damper and glass doors } \\
\text { or no fireplace. }\end{array}$ & $\begin{array}{l}\text { Poorly sealed wood stove } \\
\text { or fireplace with either well- } \\
\text { fitting damper or glass doors. }\end{array}$ & $\begin{array}{l}\text { Both a wood stove and fire- } \\
\text { place or a fireplace with } \\
\text { poorly fitting damper and no } \\
\text { glass doors. }\end{array}$ \\
\hline $\begin{array}{l}\text { Ducturork } \\
\text { and } \\
\text { noor }\end{array}$ & $\begin{array}{l}\text { No ductwork and few floor } \\
\text { penetrations or all ductwork } \\
\text { in conditioned space and } \\
\text { no floor penetration. }\end{array}$ & $\begin{array}{l}\text { Ductwork in conditioned } \\
\text { basement and few floor } \\
\text { penetrations. }\end{array}$ & $\begin{array}{l}\text { Ductwork in unconditioned } \\
\text { space and many floor } \\
\text { penetrations. }\end{array}$ \\
\hline $\begin{array}{l}\text { Vents in } \\
\text { conditioned } \\
\text { space }\end{array}$ & $\begin{array}{l}0 \text { No undampered vents and } \\
\text { fewer than three dampered } \\
\text { vents. }\end{array}$ & $\begin{array}{l}\text { Fewer than three undampered } \\
\text { vents or at least three } \\
\text { dampered vents. }\end{array}$ & $\begin{array}{l}\square \text { More than three undampered } \\
\text { vents. }\end{array}$ \\
\hline Lifestyle & $\begin{array}{l}\square \text { Fewer than six entrances and } \\
\text { exits per day. }\end{array}$ & $\begin{array}{l}\text { Six to thirteen entrances } \\
\text { and exits per day. }\end{array}$ & $\begin{array}{l}\text { More than thirteen } \\
\text { entrances and exits per day. }\end{array}$ \\
\hline
\end{tabular}




\section{POST-RETROFIT AUDIT DATA FORM}

Retrofit program name Monitoring Conservative Retrofits in Single Family Bldgs

Sponsoring organization(s)

DOE/FPL/State of FL

Experimental site desig ation JP (\#10)

Interviewer name

Date of interview $\underline{9 / 15 / 92}$

1. General information from householder

1. Name Jon Peterson

2. Street address

City State

3. Phone number

4. Own Rent

5. If householder different from that of "Pre-Retrofit Audit," or if tenure has changed, record below:

Change in householder Date of change

Change in tenure Date of change

6. Number of occupants, by age group:

\begin{tabular}{ll}
\multicolumn{1}{c}{ Age group } & Number \\
\hline Preschool & -1 \\
School age & $\frac{1}{2}$ \\
Adult, but not retired & - \\
Retired &
\end{tabular}

How many are generally home all day? Weekday

$\frac{1}{3}$

II. Significant changes since pre-retrofit audit NONE

(The remaining questions refer to the time since the energy-saving improvements were made to the house).

1. Have there been any important changes or additions to the house, such as (check appropriate): No

Rooms added Siding added

Garage/carport added Exterior paint 
Porch added New roof

Other (specify)

2. Other than changes of this program, has the main heating or cooling system been changed, such as (check appropriate): No

New furnace/boiler

New air conditioner

New heat pump

Modified furnace/boiler (specify)

Other (specify)

3. Are the same number of rooms still heated and cooled? (If not, specify) Yes

4. Have any space heaters been added/subtracted? (Fireplaces, wood stoves, electric, fossil fuel, etc.) Yes No $\mathrm{X}$

If so, specify

Has the use of these heaters changed during this last winter? Used more this winter _ Less _ Same $\mathrm{X}$ Do not know

5. Has the hot water heating system changed? Yes _ No $\mathrm{X}$ If so, specify

6. Have any major appliances been added/subtracted? (Stoves, ovens, washers, dryers, etc.) No

If so, specify

7. Is there: An attic fan No Whole house fan No

If so, has the use of these fans changed during this last summer?

Used more this summer _ Less _ Same _ Do not know

8. Other than changes of this program, have any other energy-saving changes been installed this past year? Yes __ No _

If yes, specify:

Ceiling insulation added Weatherstripping

Wall insulation added Caulking

Storm windows added Hot water flow restrictors

Storm doors added Clock thermostat

Other (specify) 
9. Have you or any family members taken up any new hobbies during the past year that require the use of large electrical or gas equipment?

Yes — No $\longrightarrow$

If so, specify 
APPENDIX E

POWER USAGE HISTORY 
ACCT NOI- 22 207340791024

NAME -- WAYNE A"LEGFIOS

SEFi ADD - 399 SE CHAFILOTTA AU

STATUS- 4/1. ACTIUE

DFITG CON-

$02 / 2 \div / 89 \quad 5 E$

STD FO - FALM EIAY FL 32909

FEV /FATE- 1/44/ FES-1

CIS ACTY-- NONE FENDITNG

5670616

$* * * * * * * * * * * * * * * * * * * * * * * * * *$ USAGE HISTOFY INFOFIMATION $* * * * * * * * * * * * * * * * * * *$ FG

CONST: KWWH1

SUC TO FEEAD DAYS FIC ECD

$02 / 26 / 92$

$01 / 28 / 92$

$29 \quad F$

1. $2 / 28 / 91$.

31

$11 / 26 / 91$

32

$10 / 25 / 91$

$09 / 25 / 91$

32

30

$08 / 26 / 91$

$07 / 25 / 91$

$06 / 25 / 91$.

$05 / 24 / 91$

$04 / 25 / 91$

$03 / 27 / 91$

$02 / 26 / 91$

30

32

30

32

2.9

2.9

29

FHONE NUMEEF 72329

ENTEF: F'

KWH KWW KIDE

$1 \times 236 \quad 19764$

1.404

1,306

1,672

1,509

1,500

$1,91.4$

2,169

2,322

1,363

1,340

1,273

1,300 $-\cdots-\cdots$

$-\cdots+-$

$-\cdots+\cdots$

- -....- -

-............-

$-\cdots+\cdots$

$-\cdots+\cdots$

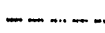

$-\cdots-\cdots$

$-1$

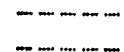

EILL.L. AMT

$$
107.25
$$

1.24.9\%

11.6 .10

149.26

J. 34.50

1.30 .83

1.69 .05

1.91 .87

205.03

121.22

1.17 .41

1.7 .58

120.13

ENTEF TO GET ADDITIONAL INFOFMATION.

ENTEF TFANSACTION CODE OF: FAGING FEQUEST

ACCT NO- 22. 207340791024

NAMIE - - WAYNE A'LEGFIOS

SEF ADD- 399 SE CHAFLLOTTA AU

OTD FO - FALM EAY FL 32909
STATUS- 4/1. ACTIUE

OFIIS CON-

FEEU/FATE- 1/44/ FIS - 1

CIS ACTY-- NONE FENDINO:
$0212 \%$ SE

5070616

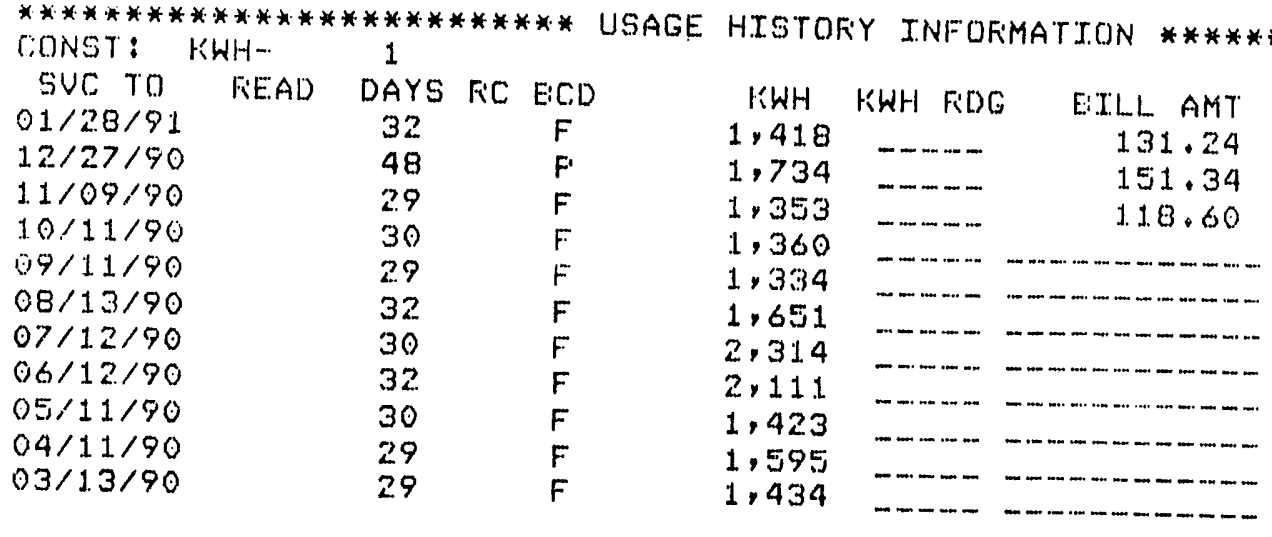

FHONE NUMEEF 723-9069

DEFFIESS FF3 TO GET FIFIST FAGE.

ENTEF TFIANSACTION CODE OF F'AGING FIEQUEST 
ACCT NO- $2109 \quad 3320988611$

NAME --- W H'SCHICK

SEF ADD- 1688 SHOFE DF

STD F'O - MEFFITT IS FL 32952
STATUS- 4/1 ACTIVE

OFIG CON-

REV/FATE- 1/44/ F'S-1

CIS ACTY-- NCNE PENDING

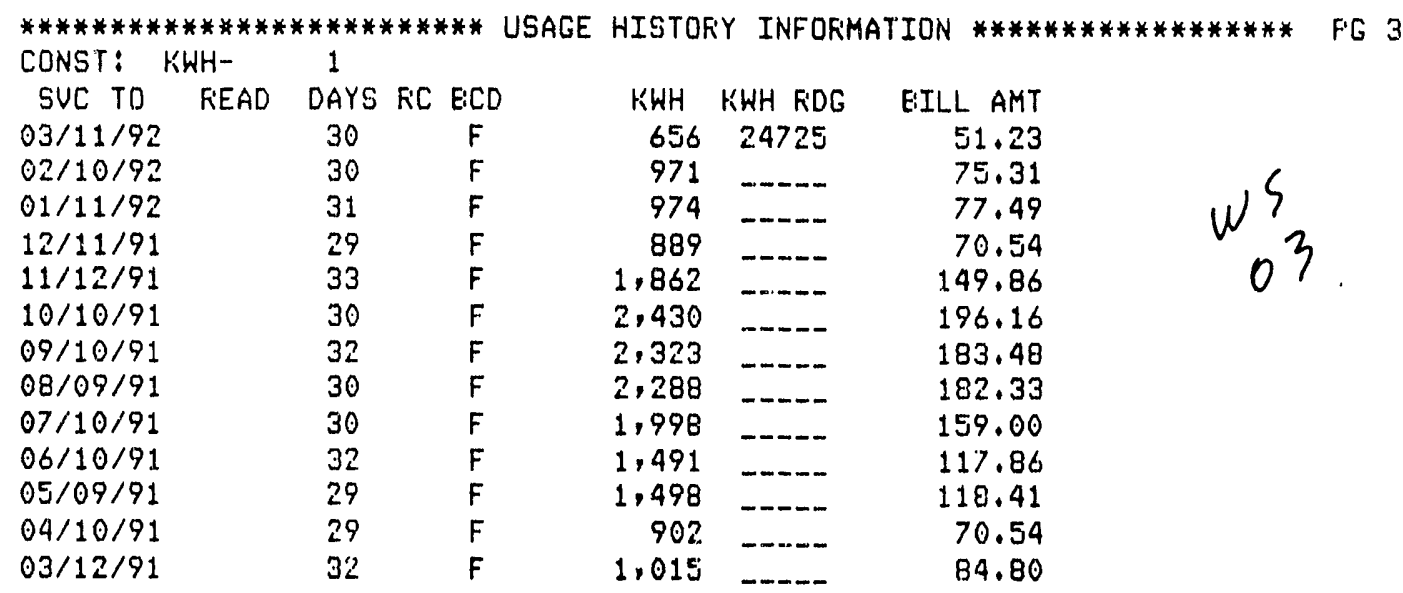

ENTEF $P / 3 / 2$ TO GET ADDITIONAL INFORMATION. ENTEK TFIANSACTION CODE OR PAGING REQUEST

ACCT NO- $2109 \quad 332 \quad 0988611$

NAME -- W H'SCHICK

SEK ADD- 1688 SHOKE DF

STD FO - MEFFITT IS FL 32952
STATUS- 4/1 ACTIVE

OFIIG CON-

REV/FATE- 1/44/ RS-1

CIS ACTY-- NDNE FENDING
$M N$

$5 C 45892$

\begin{tabular}{|c|c|c|c|c|c|c|}
\hline CONST: & KWH- & 1 & & & & \\
\hline $\begin{array}{l}\text { SUC TO } \\
02 / 08 / 91 \\
01 / 14 / 91\end{array}$ & READ & $\begin{array}{l}\text { DAYS } \\
25 \\
32\end{array}$ & $\begin{array}{c}\mathrm{FC} B C D \\
\mathrm{~F} \\
\mathrm{~F}\end{array}$ & $\begin{array}{l}\text { KWH } \\
684 \\
957\end{array}$ & KWH F'DG & $\begin{array}{r}\text { BILL AMT } \\
56.89 \\
79.84\end{array}$ \\
\hline $12 / 13 / 90$ & & 30 & $F$ & 1,073 & & 84.72 \\
\hline $11 / 13 / 90$ & & 32 & $F$ & 1,280 & & 101.43 \\
\hline $10 / 12 / 90$ & & 30 & $F$ & 1,892 & $-\ldots$ & $\ldots$ \\
\hline $09 / 12 / 90$ & & 29 & $F$ & 1,808 & & \\
\hline $08 / 14 / 90$ & & 32 & $F$ & 2,044 & .. & _ \\
\hline $07 / 13 / 90$ & & 30 & $F$ & 2.240 & & \\
\hline $06 / 13 / 90$ & & 30 & $\mathbf{F}$ & 1,778 & & $\ldots$ \\
\hline $05 / 14 / 90$ & & 32 & $\mathbf{F}$ & 1,492 & & \\
\hline $04 / 12 / 90$ & & 29 & $F$ & 922 & & \\
\hline
\end{tabular}

DEFRESS FF3 TO GET FIKST PAGE.

ENTEK TRANSACTION CODE OR F'AGING FEQUEST 
ACCT NO $22 \quad 196551206048$

NAME - - FETEK' F'OSTMA

SEF ADD - 311 COCOA AV

STD FO - INDLANTIC FL 32903
STATUS- $4 / 1$ ACTIUE

OFIG CON-

$05 / 02 / 91$

ES

REV/FATE- 1/44/ FIS-1

CIS ACTY-- NONE FENDING 5009690

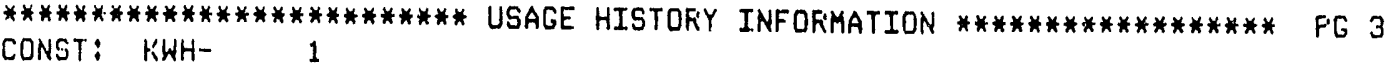

SH'C TO FIEAD DAYS FC BCD

$02 / 25 / 92$

$01 / 27 / 92$

$12 / 27 / 91$

$11 / 25 / 91$

$10 / 24 / 91$

$09 / 24 / 91$

$08 / 23 / 91$

$07 / 24 / 91$

$06 / 24 / 91$

$05 / 23 / 91$

$\begin{array}{ll}29 & F \\ 31 & F \\ 32 & F \\ 32 & F \\ 30 & F \\ 32 & F \\ 30 & F \\ 30 & F \\ 32 & F \\ 21 & F\end{array}$

\begin{tabular}{|c|c|}
\hline KWH & KWH K'DG \\
\hline 857 & 57027 \\
\hline 943 & $m-4$ \\
\hline 1,119 & \\
\hline 935 & \\
\hline 984 & \\
\hline $\begin{array}{l}2,161 \\
1,734\end{array}$ & \\
\hline 1,546 & \\
\hline $\begin{array}{r}1,327 \\
795\end{array}$ & 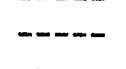 \\
\hline
\end{tabular}

EILL AMT

73.56

83.08

99.00

82.38

86.79

189.08

152.68

135.90

116.04

69.32

FHONE NUMEER 722-2414

ENTEFi TFANSACTION CODE OF FAGING REQUEST 
ACCT NO- $22 \quad 19 \quad 658 \quad 0938025$

NAME - $R$ E'RAINSEERGER

SEF ADD - 209 FLAMINGO LN

STD F'O - MELEFIN EICH FL 32951
STATUS- 4/1 ACTIVE

$09 / 05 / 67$

E:S

OFIIG CON-

REV/RATE- 1/44/ RS-1

CIS ACTY-- NONE PENDING

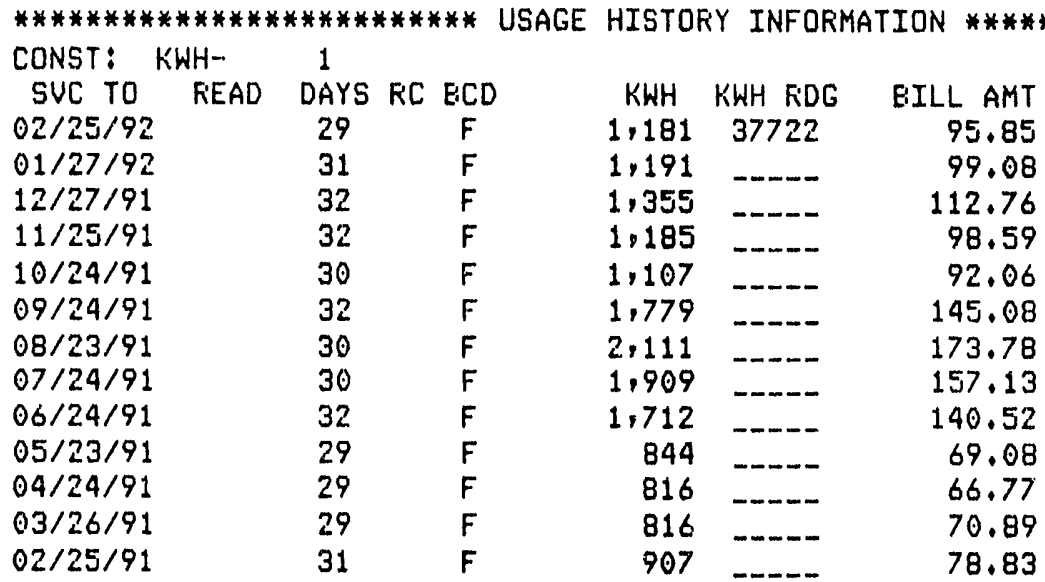

PHONE NUMBEF 723-7007

ENTER F $/ 3 / 2$ TO GET ADOITIONAL INFORMATION.

ENTER TRANSACTION CODE OR PAGING REQUEST

ACCT NO- $22 \quad 19 \quad 6580938025$

NAME -- R E"FIAINSEEFGER

SER ADD- 209 FLAMINGO LN

STD FO - MELERN BCH FL 32951
STATUS- 4/1 ACTIVE

ORIG CON-

REV/RATE- 1/44/ RES-1

CIS ACTY-- NONE PENDING<smiles>C1=CC=[Pt]=1</smiles>

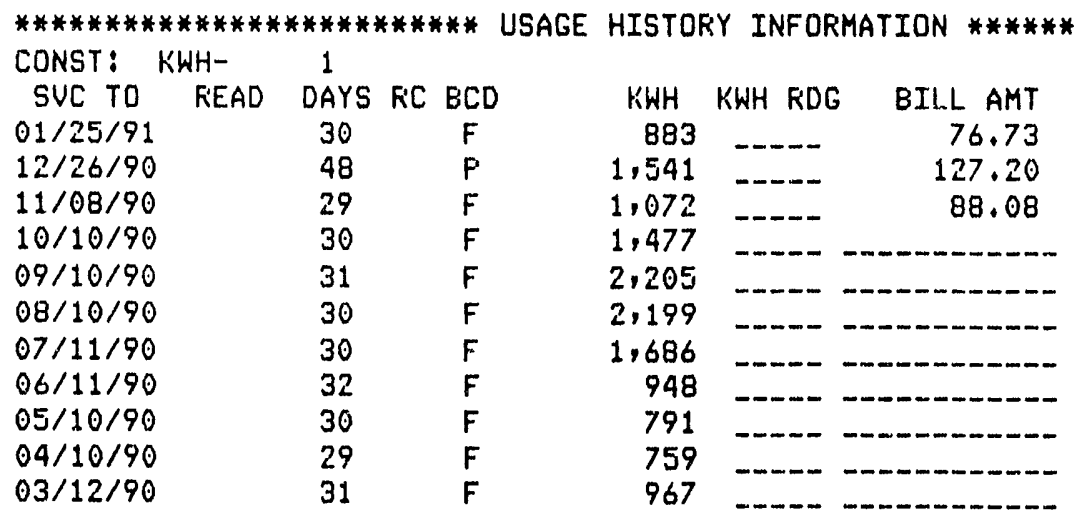

PHONE NUMBEF 723-7007

DEFRESS PF3 TO GET FIFST PAGE.

ENTER TRANSACTION CODE OR PAGING REQUEST 
ACCT NO- $22 \quad 207230115045$

NAME -- SARAH R'MILLER

SER ADD - 154 NW ABALONE RD

STO FO - PALM BAY FL 32907
STATUS- 4/1 ACTIUE

$09 / 17 / 90 \quad N W$

ORIG CON- $93 / 87$ *OL** LMIS

REV/RATE- 1/44/1 RS-1

CIS ACTY-- NONE PENDING

$5 C 36707$

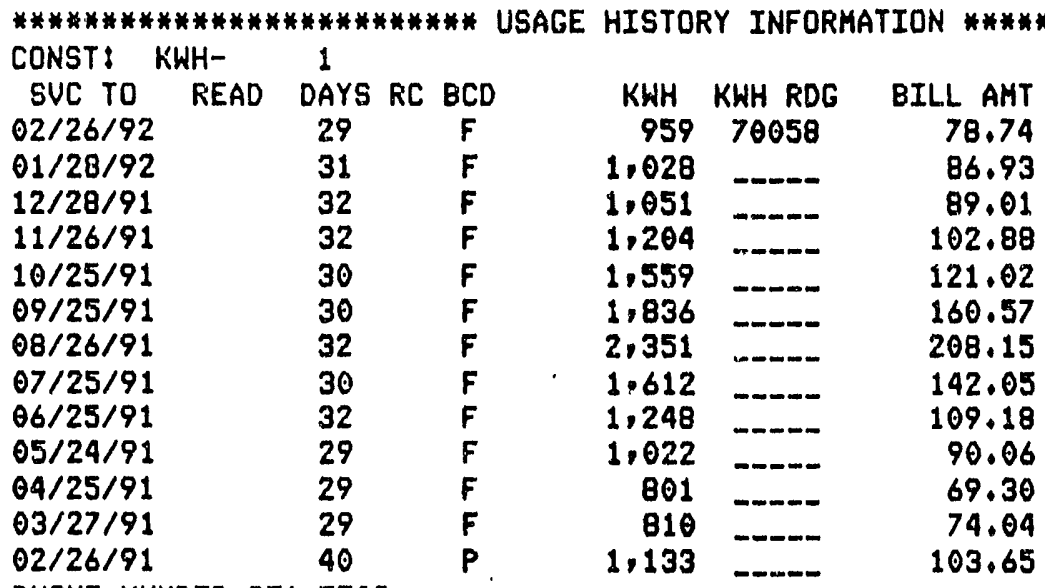

PHONE NUMBER 951-7702

ENTER P/3/2 TO GET ADOITIONAL INFORMATION.

ENTER TRANSACTION CODE OR PAGING REQUEST

ACCT NO- $22 \quad 2072301150 \quad 45$

NAME --- SARAH R'MILLER

SER ADD - 154 NW ABALONE RD

STD PO - PALM BAY FL 32907
STATUS- 4/1 ACTIUE ORIG CON- $03 / 87 * *$ *L* LHIS

REV/RATE- 1/44/1 RS-1

CIS ACTY-- NONE PENDING
$09 / 17 / 90$ NH

5036707

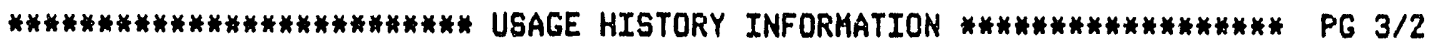
CONST: KHH-

$$
1
$$

SUC TO READ DAYS RC BCD

$01 / 17 / 91$

$12 / 18 / 90$

$11 / 02 / 90$

$10 / 03 / 90$

$\begin{array}{ll}30 & F \\ 46 & P \\ 30 & F \\ 16 & P\end{array}$

$\begin{array}{rrr}\text { KWH } & \text { KWH ROG } & \text { BILL AMT } \\ 745 & & 67.98 \\ 1,125 & \cdots & 97.25 \\ 1,273 & -\cdots & 111.45 \\ 748 & - & \end{array}$

PHONE NUMBER 951-7702

DEPRESS PF3 TO GET FIRST PAGE.

ENTER TRANSACTION CODE OR PAGING REQUEST 
ACCT NO- $22 \quad 19 \quad 656 \quad 1134016$ NAME -- E J'KIIZAK'

SEF ADD- 216 MELEIOUFINE AV

STD FO - INDLANTIC FL 32903
STATUS- 4/1 ACTIUE

ORIG CON-

F'EU/FIATE- 1/44/ FIS-1

CIS ACTY-- NONE FENOING

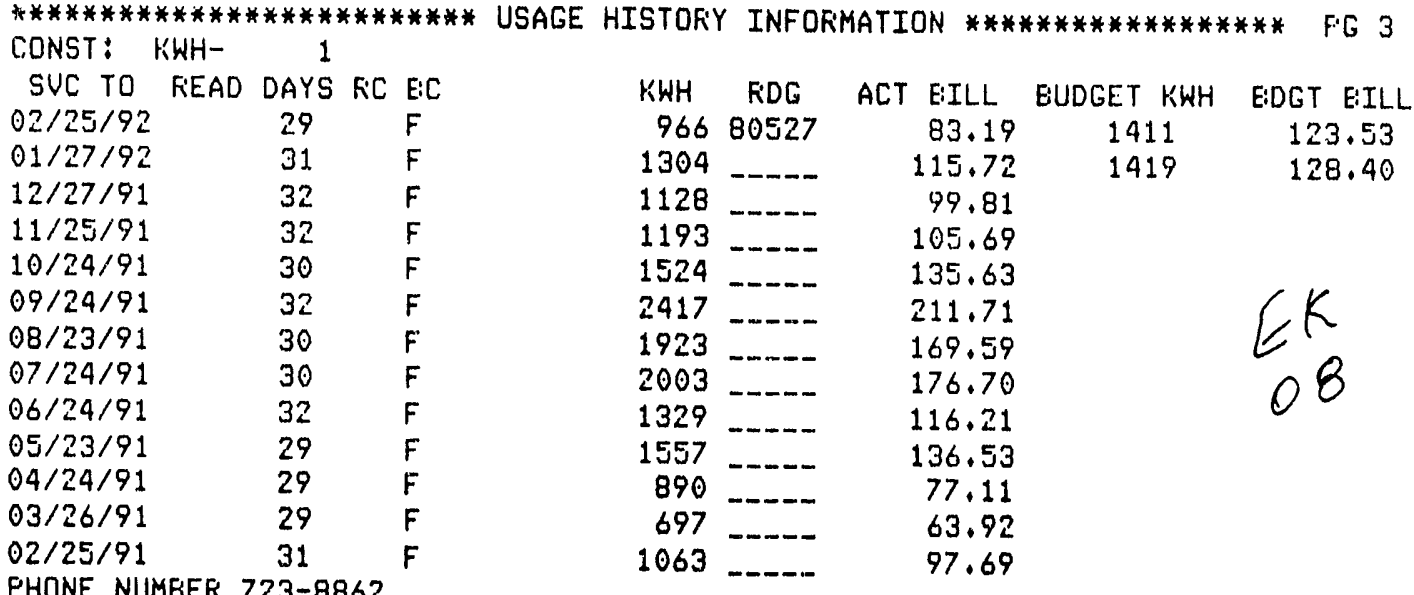

PHONE NUMBER 723-8862

ENTER $P / 3 / 2$ TO GET ADDITIONAL INFOFIMATION.

ENTER TRANSACTION CODE OR PAGING REQUEST

ACCT NO- $22 \quad 19 \quad 656 \quad 11340 \quad 16$

NAME --- E J"KIZAK

SEF ADD - 216 MELEOURNE AU

STD FO - INDLANTIC FL 32903
STATUS- $4 / 1$ ACTIUE

ORIG CON-

REV/RATE- 1/44/ FIS-1

CIS ACTY-- NONE PENDING 5C78173

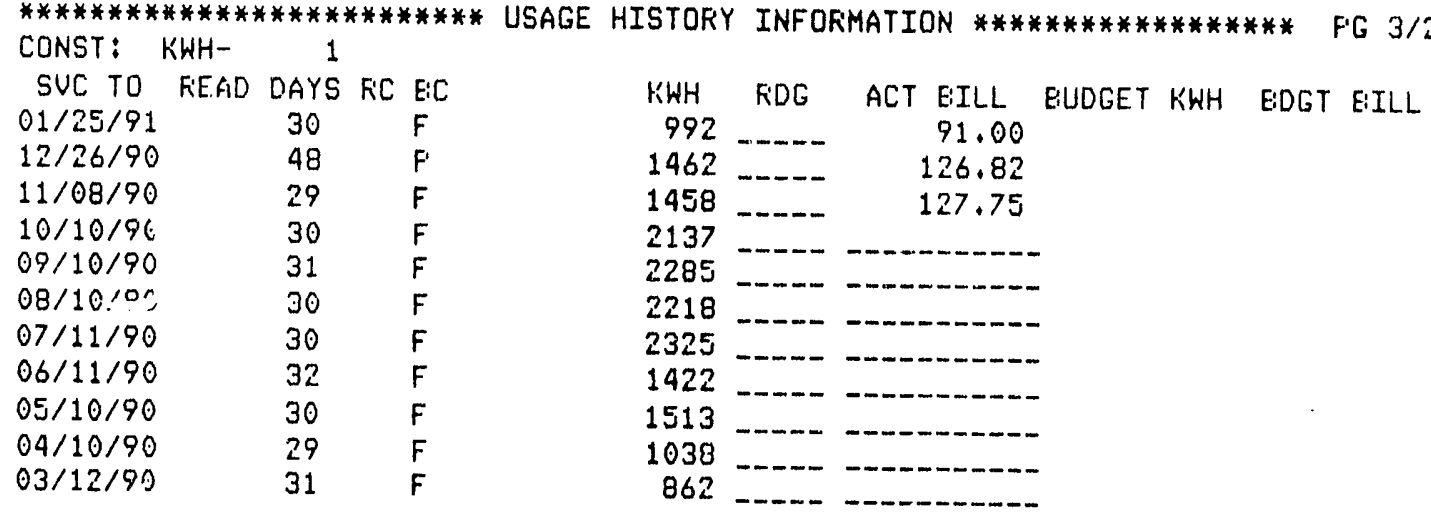

F'HONE NUMEEF 723-8862

DEFFIESS FF3 TO GET FIRST PAGE.

ENTEF TFANSACTION CODE OR PAGING REQUEST 
ACCT NO- $22 \quad 1450609620133$ KF'

NAME --- WILLIAM E'HENDLEY

SEF ADD - 2200 OHIO ST

STD FO - MELEOURINE FL 32904
STATUS- $4 / 1$ ACTIVE

ORIG CON- $08 / 73$

REEV/FIATE - 1/44/

CTS ACTY-- NONE

FiS-1

$08 / 24 / 78 \quad M W$

************************* USAGE HISTORY INFORMATION $* * * * * * * * * * * * * * * * *$ F'G 3

CONST: $K W \mathrm{WH}-$

SUC TO READ DAYS RC ECD $02 / 18 / 92$

$01 / 17 / 92$

$12 / 18 / 91$

$11 / 19 / 91$

$10 / 17 / 91$

$09 / 17 / 91$

$08 / 16 / 91$

$\begin{array}{ccc}1 \\ 32 & F A C D\end{array}$

$\mathrm{F}$
$\mathrm{F}$
$\mathrm{F}$

TWH

KWH RDG

BILL AMT

971

932

48808

72.44

29

29

30

32

$07 / 17 / 91$

$06 / 17 / 91$

$05 / 16 / 91$

$04 / 11 / 91$

$03 / 13 / 91$

$02 / 11 / 91$

$F$

$-\cdots$

77.23

966

1,327

$----$

74.06

F

1,965

$---$

76.83

106.25

-... $\quad 154.91$

125.73

$1,716 \quad 136.27$

30

F

$F \quad 1,716$

1,704

1,543

- --

134.96

121.64

857

$---m$

66.93

$1,234 \quad \ldots \quad 103.50$

$991 \ldots 2.33$<smiles>[Mg][Mg]</smiles>

F'HONE NUMEER 727-7596

ENTER $F / 3 / 2$ TO GET ADDITIONAL INFORMATION.

ENTER TRIANSACTION CODE OR PAGING REQUEST

ACCT NO- $22 \quad 14506 \quad 0962013 \quad$ RR NAME -- WILLIAM E:HENDLEY

SER ADD - 2200 OHIO ST

STD FO - MELEOUR'NE FL 32904
STATUS- $4 / 1$ ACTIVE

ORIG CON- 08/73

REV/RATE- 1/44/
$08 / 24 / 78 \quad M W$

5022615

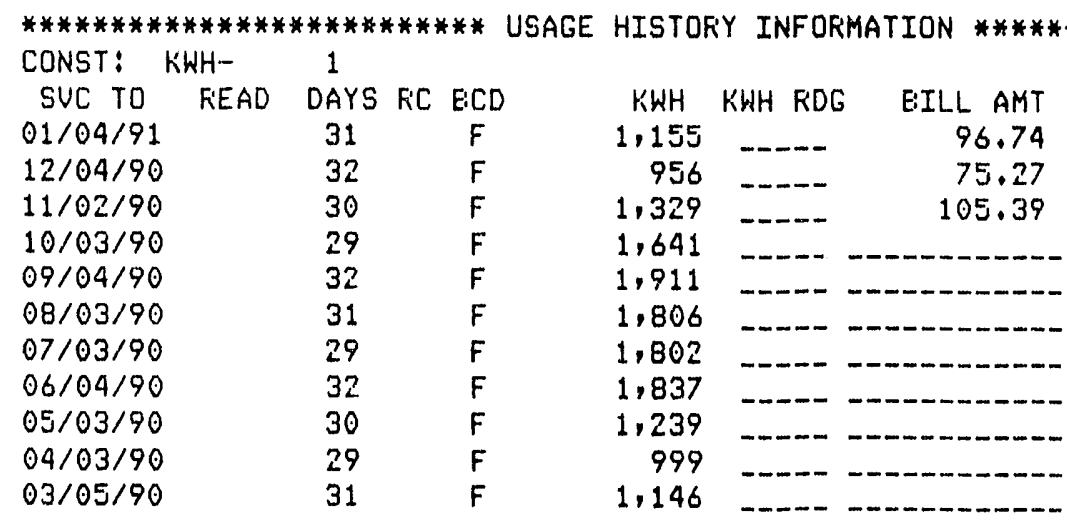

FHONE NUMEEF 727-7596

DEPRESS FF3 TO GET FIRST PAGE.

ENTER TFANSACTION CODE OR PAGING REQUEST 
ACCT NO- $22 \quad 14 \quad 4930156583 \quad$ RTi

NAME --- JON"FETEF'SON

SEF ADD - 3081 GRIACE ST

STD FO - MELEOURINE FL 32904
STATUS- 4/1 ACTIUE

$04 / 25 / 90 \quad M W$

OFIG CON- 03/90

REV/RATE- 1/44/ FiS-1

CIS ACTY-- NONE PENDING

5004687

************************** USAGE HISTORY INFORMATION $* * * * * * * * * * * * * * * * * *$ F 3

CONST: $\mathrm{KWH}-$

SUC TO READ DAYS FIC ECD

$02 / 18 / 92$

$01 / 17 / 92$

$12 / 18 / 91$

$11 / 19 / 91$

$10 / 17 / 91$

$09 / 17 / 91$

$08 / 16 / 91$

$07 / 17 / 91$

$06 / 17 / 91$

$05 / 16 / 91$

$04 / 16 / 91$

$03 / 18 / 91$

$02 / 14 / 91$

PHONE NUMBEF 951-7038

ENTER $P / 3 / 2$ TO GET ADOITIONAL INFOFMATION.

ENTEF' TRANSACTION CODE OR PAGING REQUEST

\begin{tabular}{|c|c|c|}
\hline KWH & KWH RDG & EILL AMT \\
\hline 1,000 & 68903 & 86.43 \\
\hline 963 & 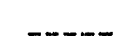 & 85.10 \\
\hline 1,008 & $--\ldots$ & 89.20 \\
\hline 1,360 & --- & 121.10 \\
\hline 1,663 & & 148.58 \\
\hline 2,133 & -3 & 187.09 \\
\hline 2.033 & & 179.86 \\
\hline 1,817 & & 160.52 \\
\hline 1,746 & & 153.78 \\
\hline 1,313 & & 115.09 \\
\hline 678 & & 59.23 \\
\hline 799 & & 73.07 \\
\hline 895 & & 83.21 \\
\hline
\end{tabular}

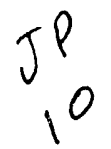

ACCT NO- $22 \quad 14 \quad 493 \quad 01565 \quad 8 \quad 3 \quad$ FiFi

NAME --- JON"F'ETERSON

SEF ADD- 3081 GRACE ST

STD FO - MELEOURNE FL 32904
STATUS- 4/1 ACTIVE

DRIG CON- 03/90

FEV/RATE- 1/44/

CIS ACTY-- NONE PENDING
$04 / 25 / 90 \quad \mathrm{HW}$

$5 C 04687$

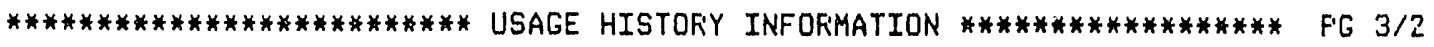
CONST: KWH- 1

SUC TO READ DAYS RC EICD

$01 / 03 / 91$

$12 / 03 / 90$

31

$11 / 01 / 90$

31

$F$

KWH

$10 / 01 / 90$

31

$08 / 31 / 90$

$08 / 02 / 90$

$07 / 02 / 90$

$06 / 01 / 90$

$05 / 01 / 90$

31
29

29

31

31

06

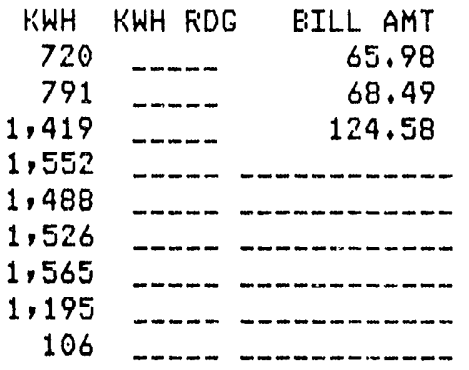

FHONE NUMEEF 951-7038

DEF'RESS F'F 3 TO GET FIRST PAGE。

ENTEF TKIANSACTION CODE OR FAGING REQUEST 

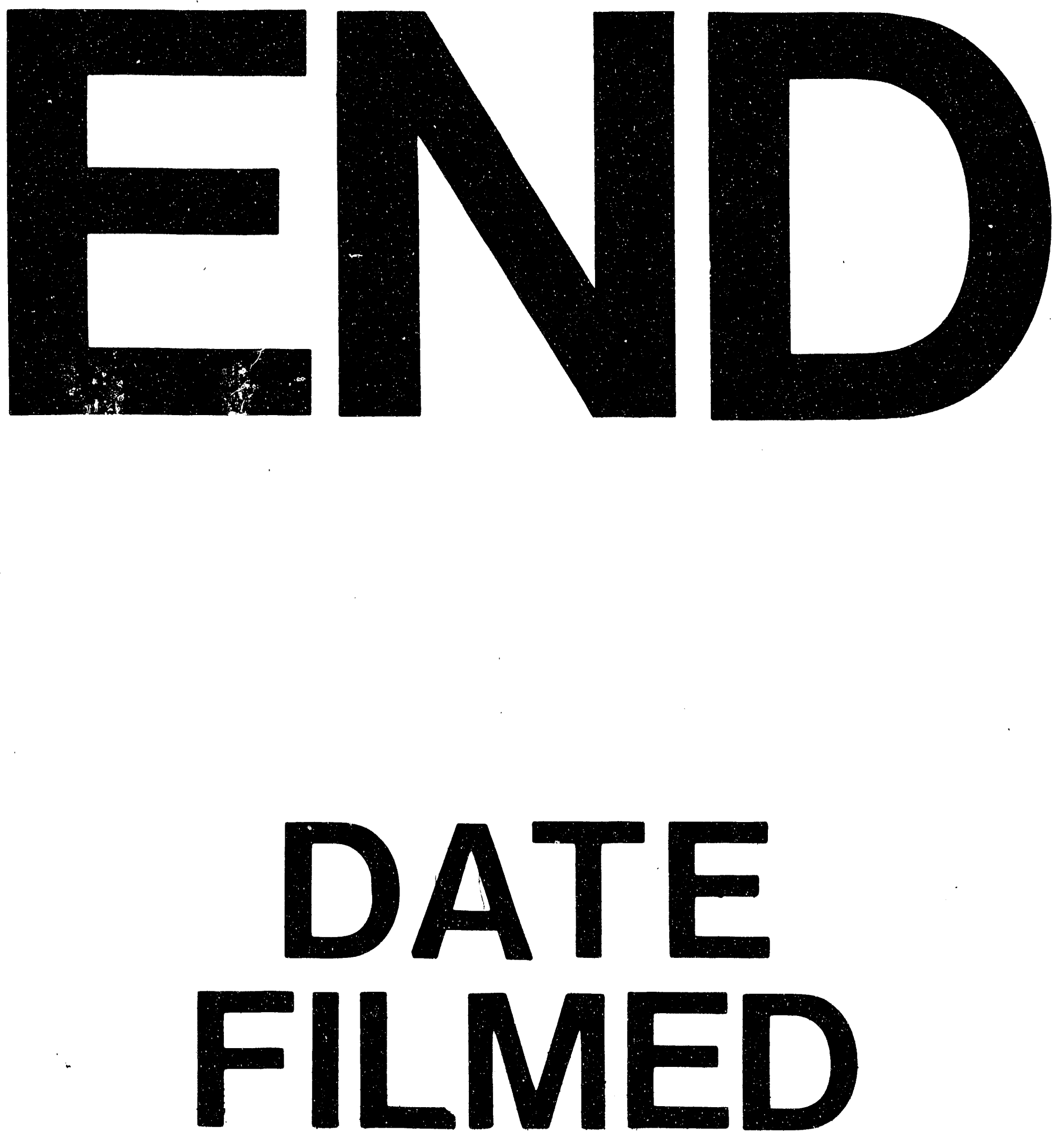

$\exists$

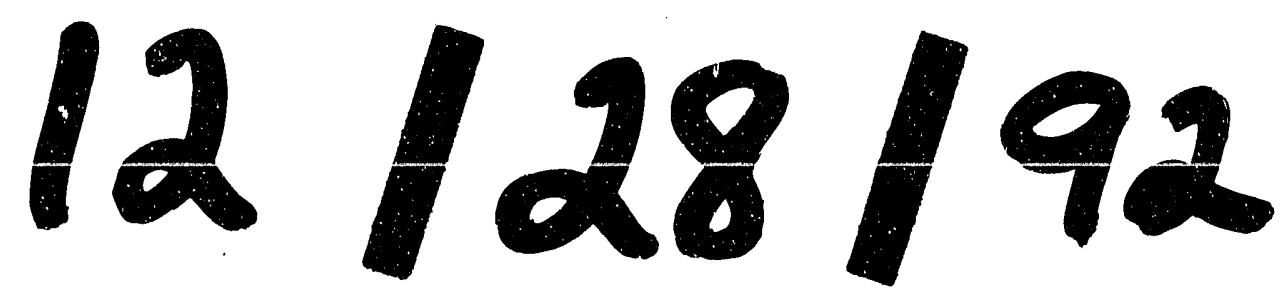


